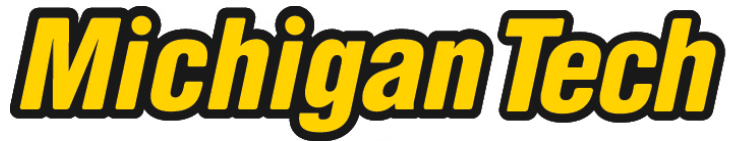 \\ Michigan Technological University Create the Future Digital Commons @ Michigan Tech
}

2014

PROOF OF CONCEPT PROTOTYPE FOR A RAILROAD PEDESTRIAN WARNING SYSTEM USING WIRELESS SENSOR NETWORKS

Puneeth Ramesh

Michigan Technological University

Follow this and additional works at: https://digitalcommons.mtu.edu/etds

Part of the Electrical and Computer Engineering Commons Copyright 2014 Puneeth Ramesh

\section{Recommended Citation}

Ramesh, Puneeth, "PROOF OF CONCEPT PROTOTYPE FOR A RAILROAD PEDESTRIAN WARNING SYSTEM USING WIRELESS SENSOR NETWORKS", Master's report, Michigan Technological University, 2014.

https://doi.org/10.37099/mtu.dc.etds/830

Follow this and additional works at: https://digitalcommons.mtu.edu/etds

Part of the Electrical and Computer Engineering Commons 


\title{
PROOF OF CONCEPT PROTOTYPE FOR A RAILROAD PEDESTRIAN WARNING SYSTEM USING WIRELESS SENSOR NETWORKS
}

By

Puneeth Ramesh

\begin{abstract}
A REPORT
Submitted in partial fulfillment of the requirements for the degree of MASTER OF SCIENCE

In Electrical Engineering
\end{abstract}

MICHIGAN TECHNOLOGICAL UNIVERSITY

2014

(C) 2014 Puneeth Ramesh 
This report has been approved in partial fulfillment of the requirements for the Degree of MASTER OF SCIENCE in Electrical Engineering

Department of Electrical and Computer Engineering

\author{
Report Advisor: $\quad$ Dr. Roger M. Kieckhafer \\ Committee Member: $\quad$ Dr. Zhaohui Wang \\ Committee Member: $\quad$ Dr. Pasi Lautala \\ Department Chair: $\quad$ Dr. Daniel R. Fuhrmann
}




\section{Table of Contents}

List of Figures...........................................................................................................................................III

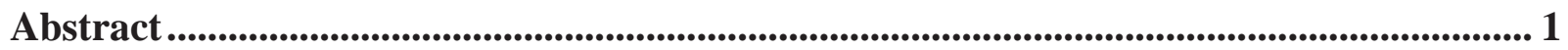

1. Introduction...................................................................................................................................... 2

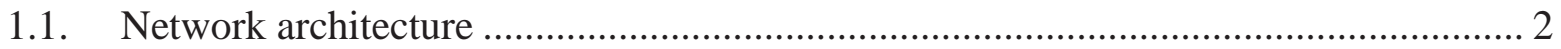

1.2. Proof-of-Concept vs. Production System ………................................................... 3

1.2.1. Sensor Subsystem............................................................................................... 4

1.2.2. Software-Configurable Radio Subsystem …………………….......................... 4

1.2.3. Annunciator Subsystem........................................................................................ 4

1.2.4. Power Supply subsystem.................................................................................. 5

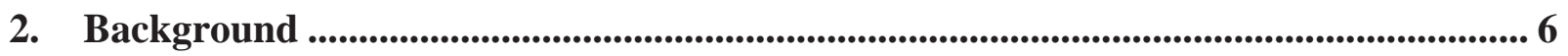

3. Basic Operation.............................................................................................................. 7

4. Hardware implementation .......................................................................................................... 10

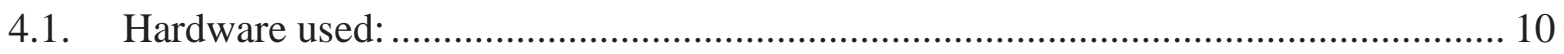

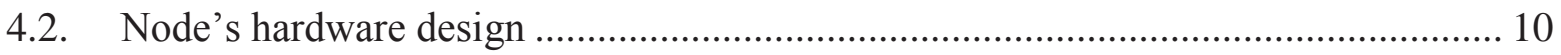

4.3. Receiver node's design .................................................................................... 12

5. Software implementation ........................................................................................................ 13

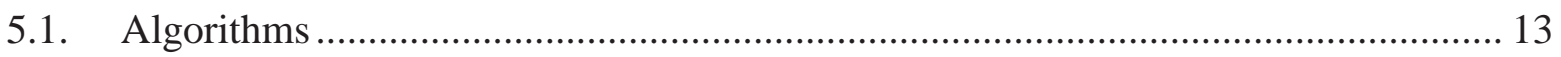

5.1.1. Procedure for clock correction ....................................................................... 13

5.1.2. Procedure for normal operating mode................................................................ 14

5.2. Receiver node software implementation ..................................................................... 20

5.3. Software development of the monitoring GUI .............................................................. 21

6. Testing and validation ............................................................................................................... 23

6.1. Steady state output for all 16 nodes....................................................................... 23

6.2. Network's response with node 2 turned off......................................................... 24

6.3. Network's response with node 2 and node 4 turned off ............................................. 25

6.4. Network's response with alternate nodes turned off .................................................. 26

6.5. Network's response with 2 adjacent nodes turned off.................................................. 27

7. Design constrains and compromises ........................................................................................ 28

7.1. Design considerations for production unit............................................................... 28

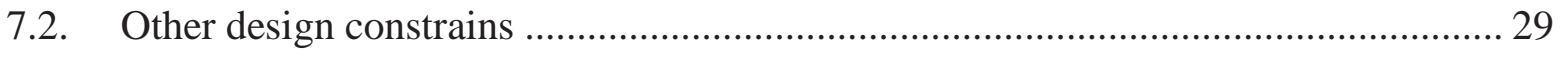


7.3. Choosing the proper frequency for communication .......................................... 29

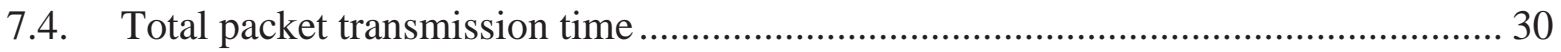

8. Conclusion ............................................................................................................. 32

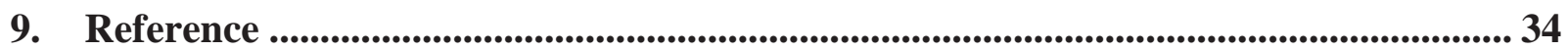

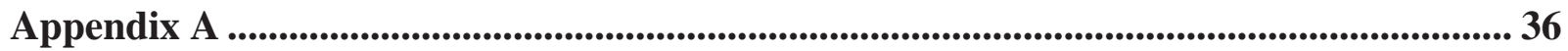

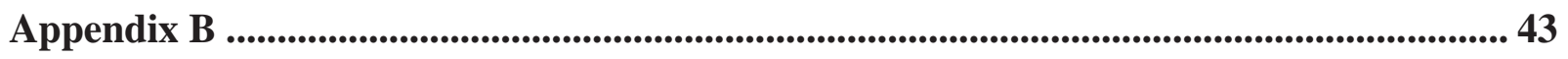

Appendix C ....................................................................................................................................... 53

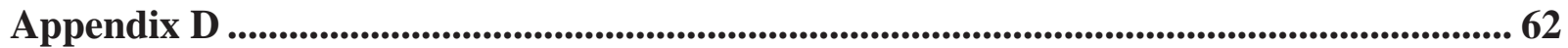

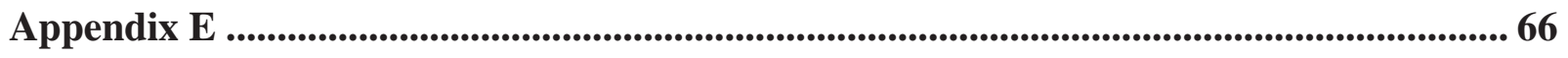




\section{List of Figures}

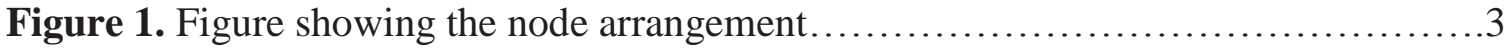

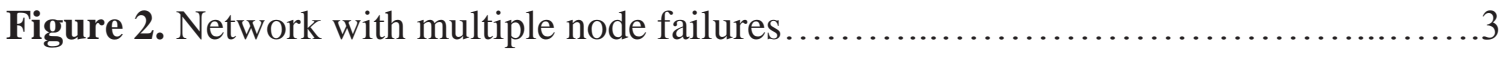

Figure 3. Figure showing one complete window.................................

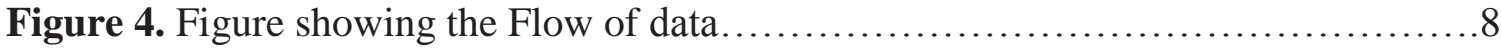

Figure 5. Operation of railway pedestrian warning system..........................9

Figure 6. Block diagram of the hardware implementation............................. 10

Figure 7. Top and front view of the prototype node................................11

Figure 8. Table showing the received packet structure............................15

Figure 9. Data stored in the next queue............................................ 16

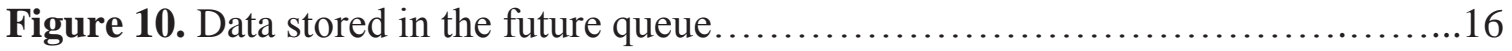

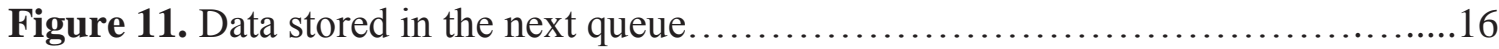

Figure 12. Data stored in the future queue.....................................16

Figure 13. Table showing the transmitted packet structure ..........................17

Figure 14. Payload Data in upstream and downstream................................17

Figure 15. Clock corrections between 2 nodes...................................20

Figure 16. Min, max and average sync offsets for all 16 nodes...........................23

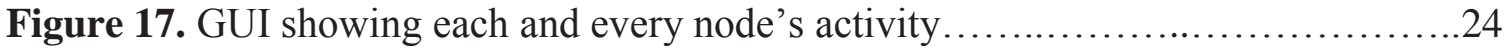

Figure 18. Table showing clock offsets for the first 5 nodes.........................24

Figure 19. Graph showing the change in offsets as node 2 is turned off................25

Figure 20. Table showing clock offsets for the first 5 nodes.........................25

Figure 21. Graph showing the change in offsets as node 2 and 4 are turned off.........26

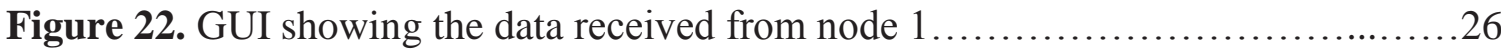

Figure 23. Min, max and average sync offsets when alternate nodes are turned off.....27

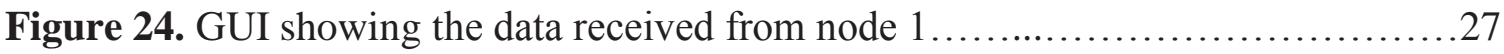

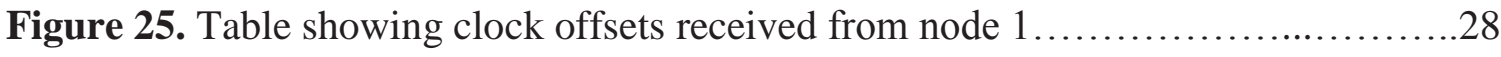

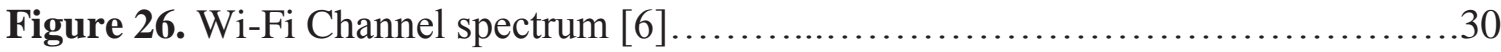

Figure 27. Node-4 communicating with 3 immediate neighbors on either side........33 


\begin{abstract}
Wireless sensor network is an emerging research topic due to its vast and evergrowing applications. Wireless sensor networks are made up of small nodes whose main goal is to monitor, compute and transmit data. The nodes are basically made up of low powered microcontrollers, wireless transceiver chips, sensors to monitor their environment and a power source. The applications of wireless sensor networks range from basic household applications, such as health monitoring, appliance control and security to military application, such as intruder detection.

The wide spread application of wireless sensor networks has brought to light many research issues such as battery efficiency, unreliable routing protocols due to node failures, localization issues and security vulnerabilities. This report will describe the hardware development of a fault tolerant routing protocol for railroad pedestrian warning system. The protocol implemented is a peer to peer multi-hop TDMA based protocol for nodes arranged in a linear zigzag chain arrangement. The basic working of the protocol was derived from Wireless Architecture for Hard Real-Time Embedded Networks (WAHREN).
\end{abstract}




\section{Introduction}

The main objective of the project was to build a proof of concept prototype to warn the pedestrians on railroad tracks about an oncoming train. In the US, on an average 500 people are injured or killed every year due to pedestrian railroad accidents [11]. Most of these accidents happen in blind spots or due to negligence. A warning system can potentially warn victims from oncoming trains. The warning systems should be capable of functioning even in areas such as tunnels, canyons and bridges.

\subsection{Network architecture}

This prototype uses a Time Division Multiple Access (TDMA) based wireless sensor network protocol based on the concepts of Wireless Architecture for Hard Real-Time Embedded Networks (WAHREN) [1]. This protocol uses peer-to-peer communication. The data from each node is relayed to its neighbors until it finally reaches the end node via a multi-hop relay. The nodes are arranged as shown in figure 1. This arrangement allows each node to communicate with two of its immediate neighbors on either side, thus providing a fault tolerant way of relaying data. Even if there is a single node failure on either side of a node the data still gets transferred. The data from each node will be available at the end nodes. For example in figure 1 data from all the nodes can be read at either node 1 or node 7. The protocol uses a TDMA based approach where each node is assigned a time slot for transmitting. The nodes use their time slots to either send their own data or to relay data from neighboring nodes. The data is transferred in systolic broadcast method.

Basic functions of the production system:

- In the production system, all nodes are equipped with sensors to detect a train, while minimizing false detection due to natural phenomena or pranksters.

- Each detection is relayed peer-to-peer to all nodes

- Each node can decide whether to issue an alert based on the distance (number of hops) to the nearest detection.

The prototype was built using a eZ430-RF2500 [2] development board from Texas Instruments (TI). The development tool has a MSP430F2274 [3] microcontroller and 
CC2500 2.4-GHz wireless transceiver [4]. To demonstrate the proper working of the protocol each node is equipped with a Passive Infrared motion sensor (PIR sensor). The status of each node's sensor is relayed to the end nodes. The prototype software was developed to support sixteen nodes; however production software could support many more.

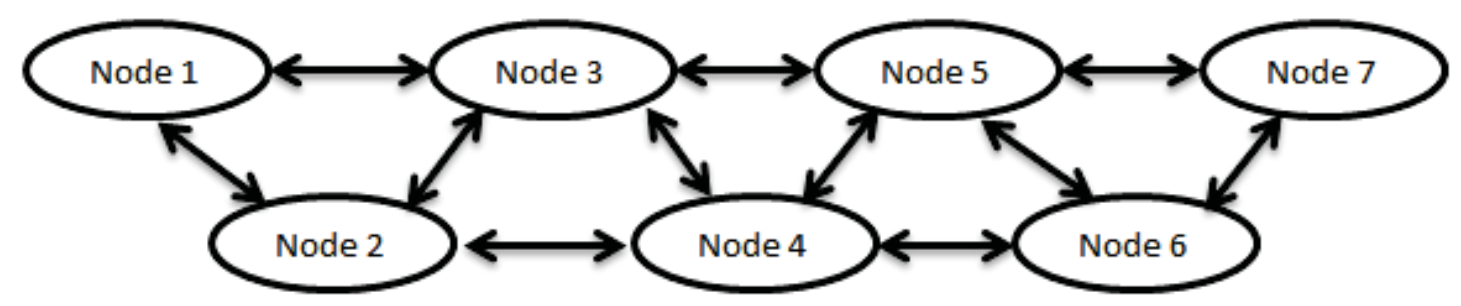

Figure 1. Figure showing the node arrangement.

The protocol is fault tolerant to single node failures at random locations. The network can still reliably route data from one end to the other even with multiple single node failures at random locations. For example, in figure 2, if nodes 3 and 6 fail, data is still reliably routed from node 1 to node 7 through nodes 2, 4 and 5.

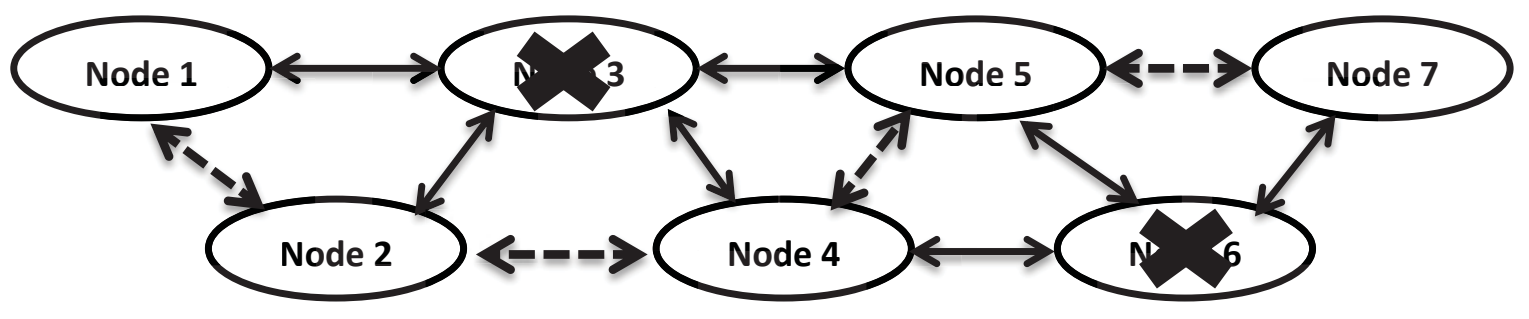

Figure 2. Network with multiple node failures.

\subsection{Proof-of-Concept vs. Production System}

The proof-of-concept system presented herein comprises the same basic subsystems and functions as would be needed in a production system. Although it varies considerably in the details of how the subsystems are implements, all of the principles are transferable and adaptable to the needs of a production system.

A production system would be required to detect a train, forward that data a mile or more down the track, and issue an audio/visual alert sufficient to attract a trespasser's attention well in advance of the arrival of the train. The proof of concept system is a much smaller 
system, designed to detect motion of a human being in a building, forward that data up-to 100 meters down the hallway, and issue a visible alert to a researcher who is looking for it.

The system comprises four basic subsystems: a Sensor subsystem, a SoftwareConfigurable Radio subsystem, an Annunciator subsystem, and a Power Supply subsystem. These subsystems are modular, and can be independently modified or adapted to in-the-field requirements and conditions.

\subsubsection{Sensor Subsystem}

The proof-of-concept system employs a simple Passive Infra-Red (PIR) sensor to detect motion of a human. By contrast, the production system would require a more sophisticated suite of sensors to detect a train, while being insensitive to false alarms, especially those induced by pranksters. Development of the production sensor suite is beyond the scope of this study.

\subsubsection{Software-Configurable Radio Subsystem}

The Radio subsystem comprises a low-cost, low-power micro-controller and an RF transceiver. Operation of this subsystem is completely dictated by the micro-controller software.

The definition and operation of the entire network is completely controlled by the microcontroller software, which can be easily adapted to any production system requirements. The primary focus of this study is demonstrating the feasibility of the network for peer-to-peer forwarding detection data to all nodes in the system.

The proof-of-concept transceiver operates in the unlicensed $2.4 \mathrm{GHz}$ band, whereas a production model would employ RF bands licensed to the railroads.

\subsubsection{Annunciator Subsystem}

The proof-of-concept Annunciator is simply an LED visible to a passer-by. A Production model subsystem would need to be bright, loud, and annoying enough to catch the attention of a distracted trespasser (e.g. one who is texting on a smart-phone while wearing earphones 
and a hooded parka). Development of the production annunciator is beyond the scope of this study.

\subsubsection{Power Supply subsystem}

Each proof-of-concept node is powered by a pair of AAA batteries. However, a production model would require a self-contained renewable power supply. Several alternatives exist, including solar, wind, and/or vibration-based sources. One advantage of this system is that the vast majority of power is consumed when a train is present. Development of a production Power Supply subsystem is beyond the scope of this study. 


\section{Background}

The routing protocol implemented in the prototype was derived from WAHREN [1]. The WAHREN routing protocol was designed specifically for highly reliable message delivery over fixed networks and for hard real-time deadlines [1]. This protocol was designed for linear topologies like figure 1 . This ideally suits the requirements for sensor placement along the railroad tracks, since they are essentially a fixed linear topology.

The WAHREN protocol was also designed to deliver messages in fixed time, where the delays for all packets are time bounded [1]. This is also a very important requirement for pedestrian warning systems. The warning message has to reach the pedestrian in time; hence delivery time of the packets is critical. The protocol uses a TDMA based approach for on time delivery of a node's packet and for fairness of medium access. WAHREN was also designed to withstand single node failures at random locations. This helps reliably route data from one end to the other even in the presence of faults [1]. The protocol also works well with gentle curves in the topology, and can be adapted to sharp corners. 


\section{Basic Operation}

This section will describe in detail the working of the protocol. The operation of each node can be divided into two modes: time synchronization mode and normal mode. The protocol is based on TDMA, hence time synchronization is important. Each node is given its own time slot. There are sixteen time slots in one window, where each time slot is $4 \mathrm{~ms}$. Hence the entire window will be $64 \mathrm{~ms}$ as shown in figure 3.

When each node is powered on, it goes through the time synchronization mode, and once it is done with time synchronization, it enters the normal mode and remains there indefinitely. During the time synchronization mode, each node synchronizes its clock to its neighbors clocks and picks a node address (which corresponds to an available time slot). When the first node is turned on it has no neighbors, so it does not make any corrections to its clock counts and just picks the first time slot. But when the second node is turned on, it first checks to see if there are transmitting nodes, picks an available time slot, and then synchronizes with the first node's clock. Once synchronized, it starts transmitting. Once node 2 starts transmitting, node 1 detects the presence of node 2 and it also makes corrections to its clock counts. This helps bring the clock counts of both the nodes as close as possible. Now, if node 3 is turned on, it does the same; it first synchronizes with both nodes 1 and 2 and then starts transmitting. All the nodes turned on follow the same procedure. Once each node synchronizes, it enters normal mode; this is when it starts transmitting.

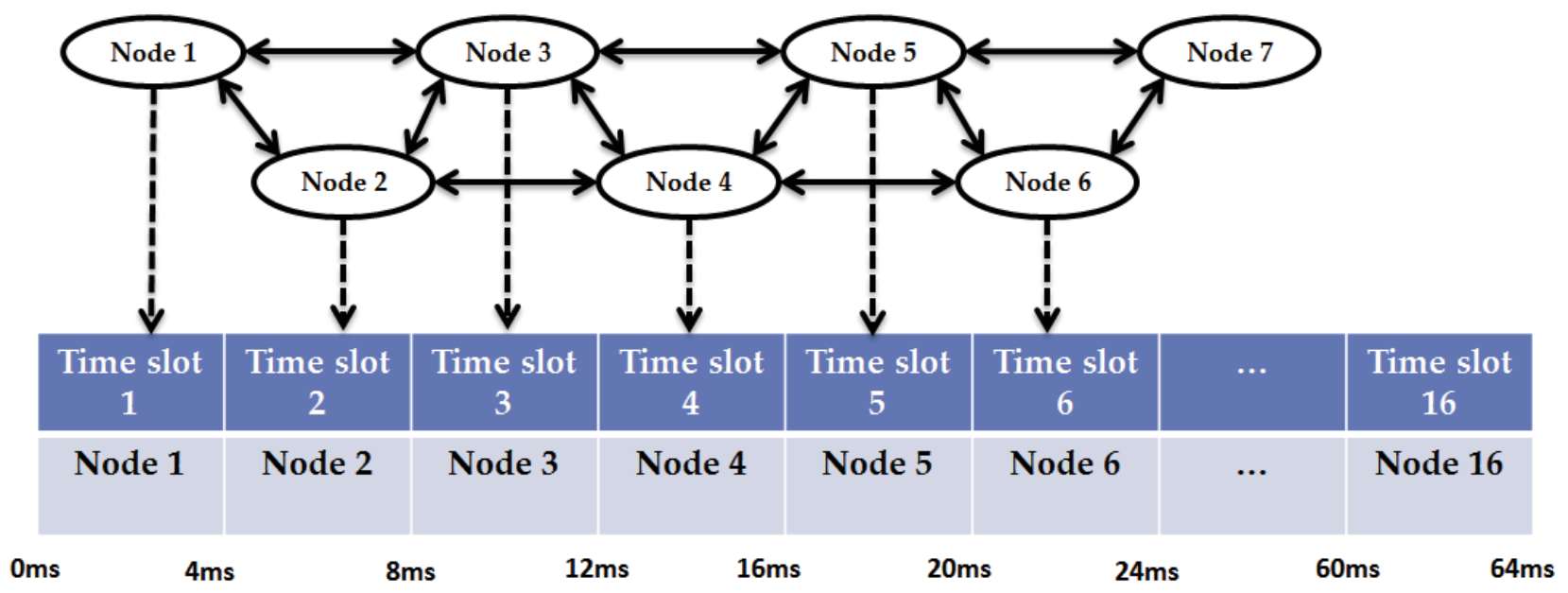

Figure 3. Figure showing one complete window. 
When a node enters normal mode it does three operations:

(1) During its time slot it transmits its data or relays data received from its neighbors.

(2) During the remaining time slots it receives data from its immediate two neighbors on either side.

(3) And at the end of each window, it performs clock count corrections based on the arrival times of its neighbor's packets.

The node remains in a low-power sleep mode in between these operations. Each node will transmit its data at the middle of its time slot; for example, node one will transmit its data at 2 msec (its time slot is from $0-4 \mathrm{msec}$ ) and node 2 will transmit its data at 6 msec (its time slot is from $4-8 \mathrm{msec}$ ), and so on. Figure 1 , shows that each node can communicate with two of its neighbors on either side. This ensures that even if one node fails, data is still relayed to the end nodes. The data payload of each node is 4 bytes, where 2 bytes carry the upstream data and 2 bytes carry the downstream data. This ensures that the same data is available at both the ends.

The prototype consists of sixteen nodes. Each node transmits its own sensor data every sixteenth window (during Window-0) and they continuously relay data received from their

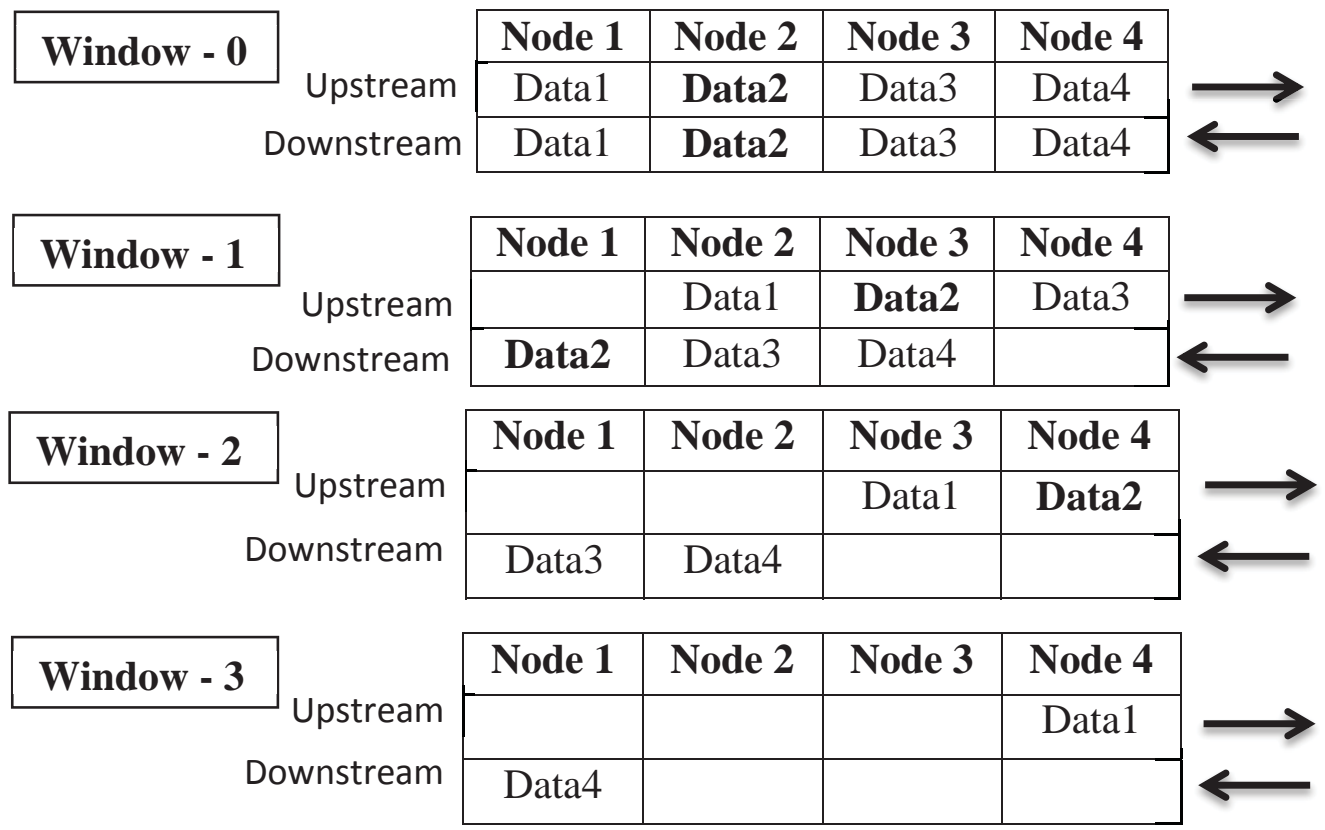

Figure 4. Figure showing the flow of data. 
during the remaining fifteen windows (Window-1 to Window-15). Figure 4 illustrates how data gets relayed to the end nodes. Each node transmits its own data during window- 0 . This is later on relayed by its neighbors to the end nodes. For example, Node 2 transmits its data ("Data2") during its time slot in Window-0. This is later on relayed by Node 3 and Node 1 in Window-1 and by Node 4 in Window-2.

When each node receives a packet from its neighbors it records the arrival time of the packet. For example in figure 1, node 1 can receive packets from both node's 2 and 3 and records the arrival time of the packets from both nodes. The difference in the expected arrival time and the actual arrival time is used to find the error in the clock counts. The average error is used to correct the nodes clock counts. This correction is done at the end of the window. The MSP430's clock is not sourced from a crystal oscillator hence the clock generators are neither exceptionally accurate nor stable; hence the clock counts of all the nodes cannot be perfectly synchronized. There will be a non-zero offset while making corrections.

In the pedestrian warning system, each and every node is equipped with a sensor to detect the presence of a train. As soon as a train is detected the warning signal is relayed ahead of the train as shown in the figure 5. The warning signal is forwarded to $\pm \mathrm{N}$ nodes from the detection; the distance to forward the alert can be dependent on the speed of the train. The distance to forward the alert should also provide the pedestrian enough time to move away from the tracks. In the image shown in figure 5 the warning signal reaches the pedestrian before the train does, providing the pedestrian enough time to move away from the tracks.

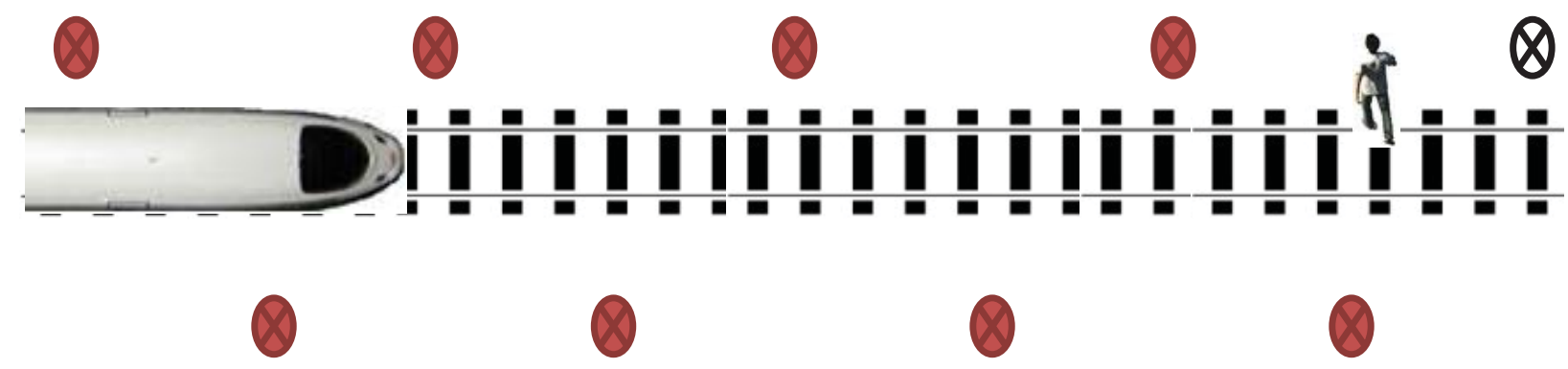

Figure 5. Operation of railway pedestrian warning system. 


\section{Hardware implementation}

\subsection{Hardware used:}

1. EZ430-RF2500 development board [2].

> MSP430F2274 microcontroller [3].

$>$ CC2500 2.4-GHz wireless transceiver [4].

2. PIR Sensor (\#555-28027) from parallax [16].

3. LED for indication.

\subsection{Node's hardware design}

This section describes in detail the hardware implementation of the prototype. The eZ430-RF2500 development board was used to implement the controller and for wireless communication. The eZ430-RF2500 is an MSP430 wireless development tool. The development board features a MSP430F2274 microcontroller and CC2500 2.4-GHz wireless transceiver [2]. The IAR embedded workbench [17] was used to program and debug the MSP430F2274 microcontroller. The schematic of the prototype is shown in Figure 6 and actual images of the prototype's top and front views are shown in Figure 7.

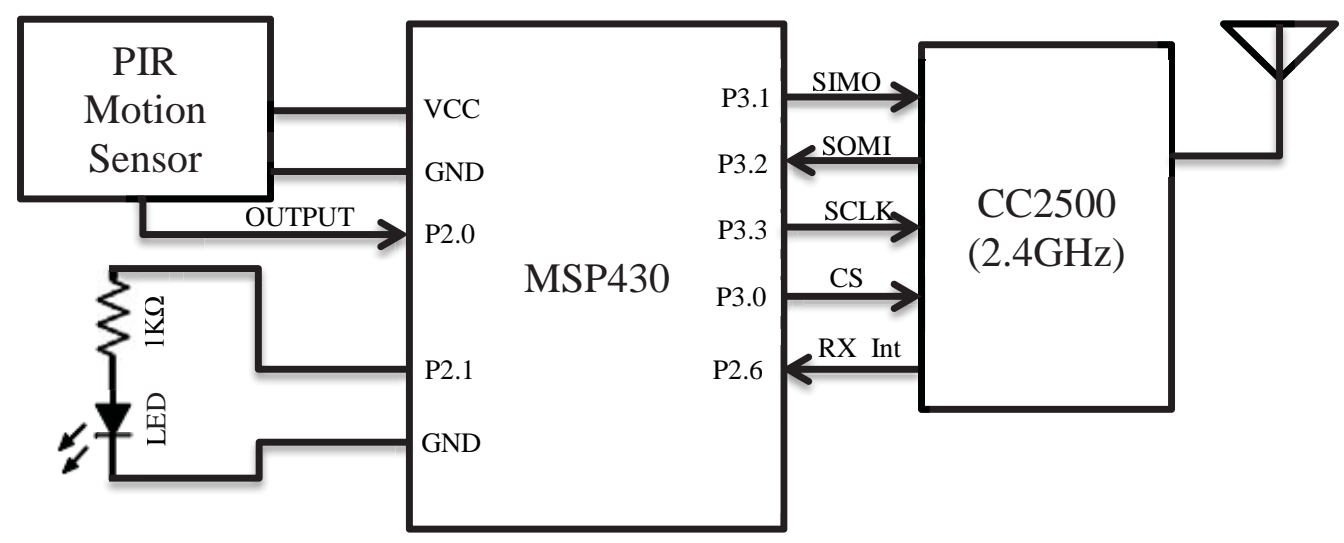

Figure 6. Block diagram of the hardware implementation. 


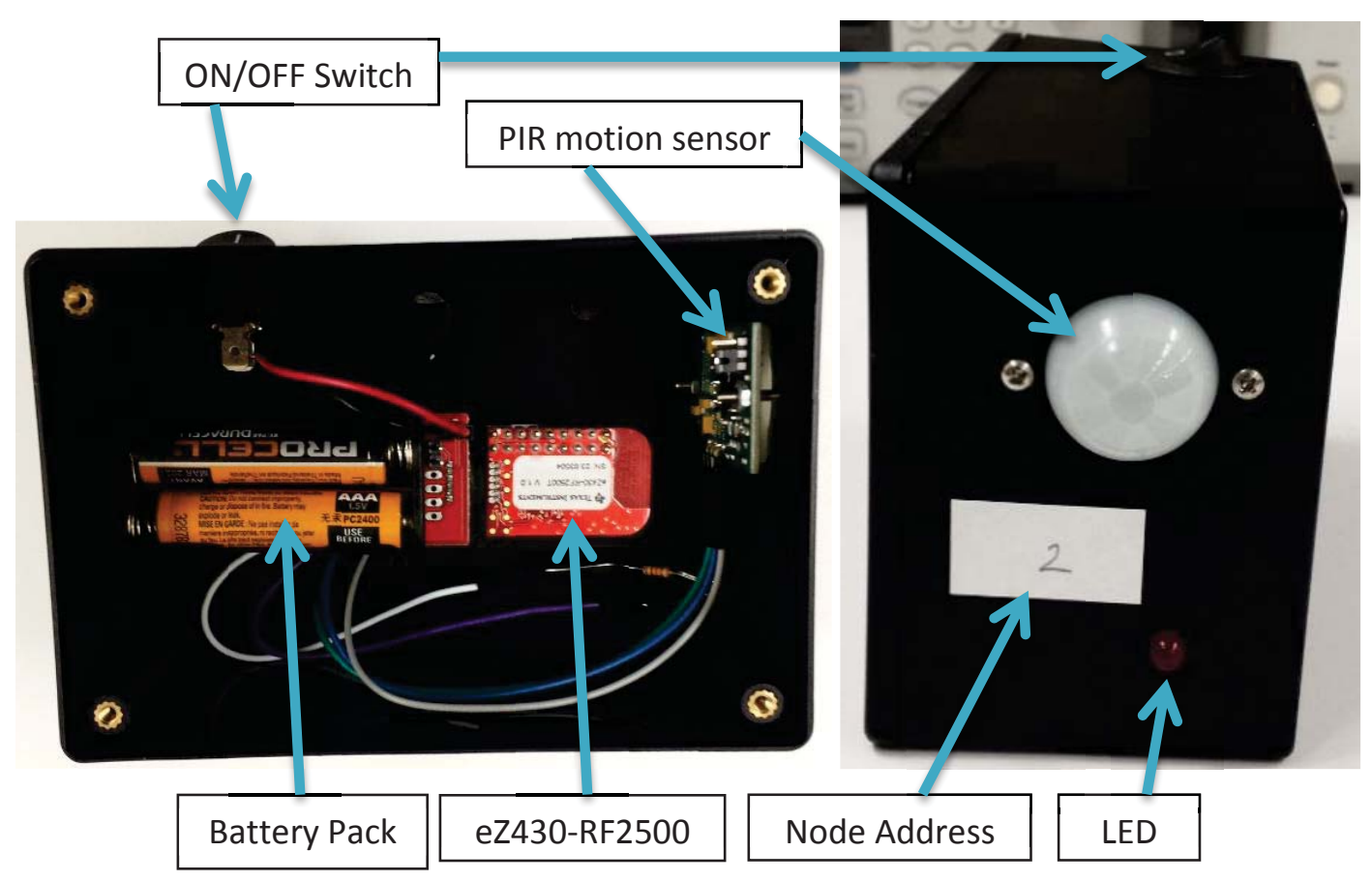

Figure 7. Top and front views of the prototype node.

The MSP430F2274 is 16-bit RISC architecture rated to operate between 1.8V and 3.6V. The prototype is powered by 2 AAA batteries. The MSP430's internal clock can be configured to run at speeds up to $16 \mathrm{MHz}$ [3]. For our application the master clock (MCLK) is configured to run at $8 \mathrm{MHz}$. The MCLK is the clock source for the CPU and a sub-master clock (SMCLK), configured to run at $1 \mathrm{MHz}$. SMCLK is the clock source used for the timers and for SPI communication with the CC2500. The timer is configured in UP mode. It is programmed to generate an interrupt at the end of each window, which is 64 msec. The timer is also used to trigger an interrupt during the node's transmit time slot. The node transmits its data during this interrupt. MSP430 external port pin P2.0 is used to read the sensor status, and port pin P2.1 is used to toggle the external LED, to signal an alert.

The MSP430 uses SPI to communicate with the CC2500 transceiver. Pins (P3.0 to P3.3) are configured for SPI communication. P3.0 is used as chip select, P3.1 is used as slave in master out (SIMO), P3.2 is used as slave out master in (SOMI) and P3.3 is used as clock source (SCLK) from master to slave. Pins P3.0 to P3.3, P2.6 and P2.7 are internally connected to the CC2500. The SPI's clock source is derived from the SMCLK. This is further divided by 2 resulting in an SPI data rate of 500 Kbps. Pins P2.6 and P2.7 are 
connected to the CC2500's GDO0 and GDO2 pins. These pins are user configurable pins, used to configure the CC2500 to generate an interrupt when a packet is received. Once SPI is configured, the CC2500 can be configured with the required settings.

The CC2500 is configured to transmit at a data rate of $250 \mathrm{Kbps}$. The CC2500 transmits in the $2.4 \mathrm{GHz}$ frequency band. The base frequency starts from $2.433 \mathrm{GHz}$. The channel spacing is configured to $199.95 \mathrm{Khz}$. The frequency has to be picked carefully, since Wi-Fi also uses the same frequency band. For our demonstration specifically channel number 89 was used. This corresponds to a carrier frequency of $2450.79 \mathrm{MHz}$.

$$
\begin{aligned}
\text { Carrier frequency } & =\text { Base frequency }+(\text { Channel spacing } * \text { Channel number }) \\
& =2.433 \mathrm{GHz}+(199.95 \mathrm{Khz} * 89) \\
& =2450.79 \mathrm{MHz} .
\end{aligned}
$$

This frequency falls into the gap between Wi-Fi channels 6 and 11. The CC2500 is configured to transmit a 4 byte preamble and a 32 bit sync word before transmitting the payload. The payload transmitted is 7 bytes long. This is appended with a 16 bit CRC for error detection. The CC2500 has on-chip support for CRC handling and sync word detection [4]. Minimum shift keying (MSK) is used to modulate the data transmitted. Carrier sense is disabled, and the GDO0 pin is configured to generate an interrupt whenever a valid packet is received. The data is transmitted as a broadcast to all nodes in the neighborhood. Data transmitted with a receiver address of $0 \mathrm{xFF}$ is considered a broadcast. The actual address of the transmitting node is included in the payload.

\subsection{Receiver node's design}

The receiver node used to collect data from any node in the network consists of the MSP430F2274 and the eZ430's USB debugger card. The receiver node collects data from a targeted node and sends it to a host computer via UART (universal asynchronous receiver/transmitter). The receiver node is configured to receive packets at the same frequency and modulation as the network nodes. The receiver node is configured to send the received packets to the host over UART at 9600 bauds with no flow control, no parity check and with 1 stop bit. 


\section{Software implementation}

\subsection{Algorithms}

This section will describe in detail the software development for the prototype.

\subsubsection{Procedure for clock correction}

The first thing that has to be done before starting to transmit is to synchronize clock counts with the neighboring nodes. This is done using the startup sync function "startup_sync()". This function makes sure that the node's clock count is as close as possible to its neighboring nodes and also assigns a node address. In this prototype, the node address is hardcoded for each node. To achieve time synchronization, each node's clock counts have to match the neighbor's clock counts as closely as possible. This is done for 16 windows. The window size is not updated now; this will be done once transmission starts. During startup sync the node will not be transmitting, it only listens to other nodes. The code for updating the clock counts to match the neighbors clock counts is shown below. The flow chart can be found in Appendix A (flow chart 4).

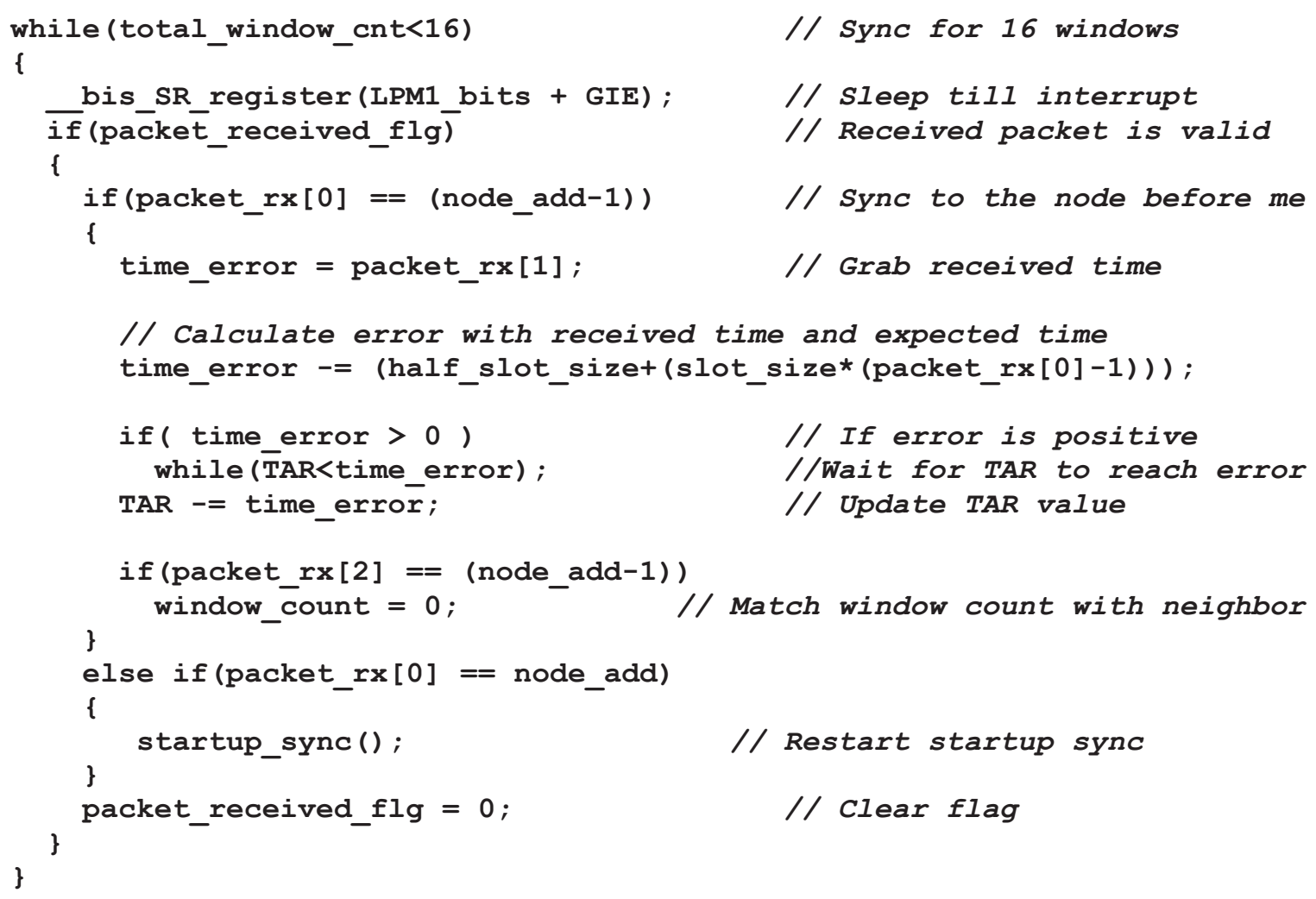


The "startup_sync()" function also matches the window count with its neighbor (this helps the node decide when to transmit its data). At the end of this function the node address is fixed and the clock error is as small as possible. Before the node starts transmitting just to make sure that the sync was proper, each node performs a sync check using the function "sync_check()". This function makes sure that the startup sync went on smoothly. It also makes sure that the error was minimized before starting to transmit; this is done for 3 windows. If the error is large then the function again calls the startup sync. The code uses a function to find the minimum error; this function's working will be discussed later. The code is shown below and the flow chart can be found in Appendix A (flow chart 5). Once done with startup sync and sync check, the node can start transmitting data.

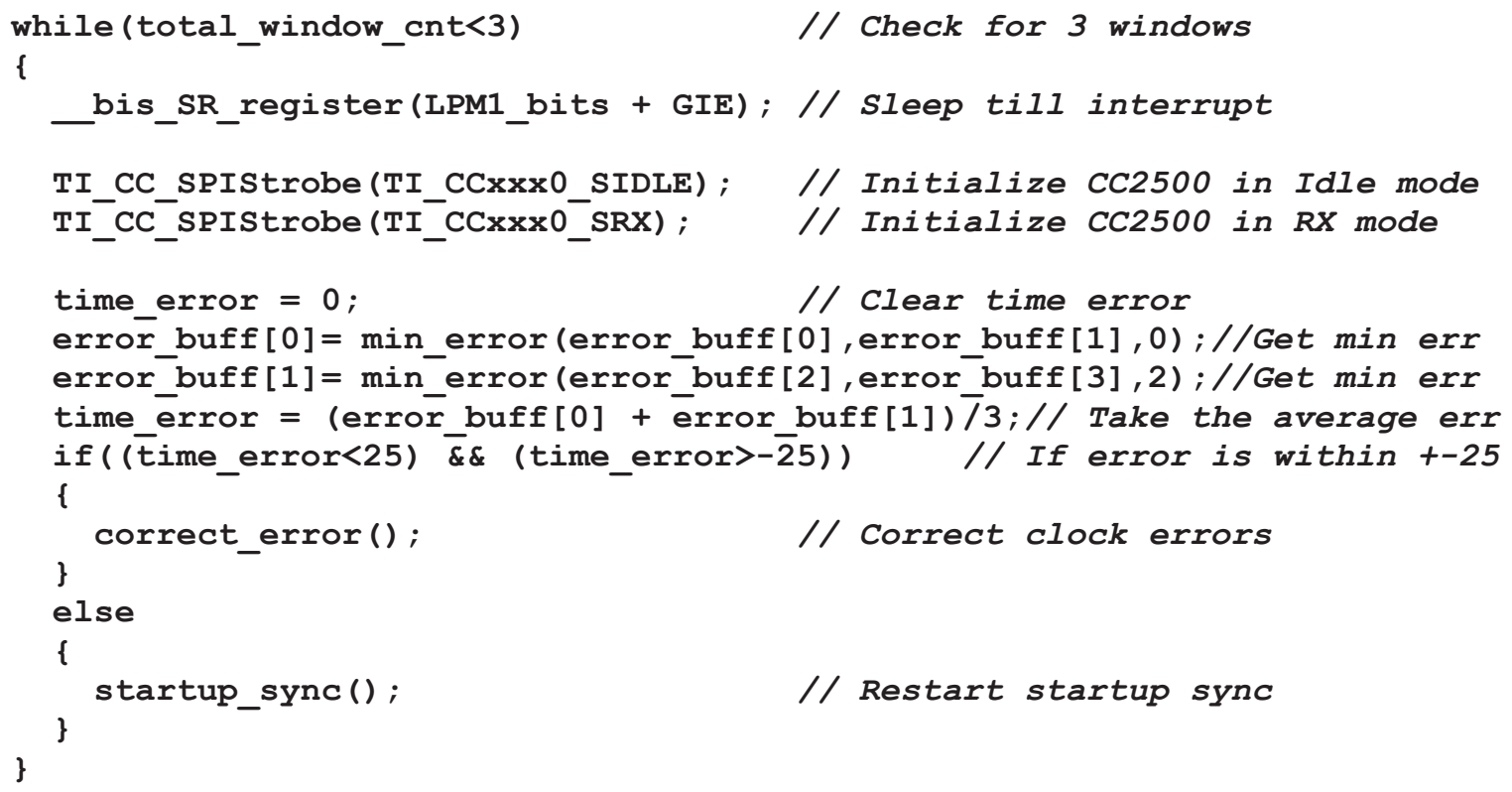

\subsubsection{Procedure for normal operating mode}

The node enters the normal operating mode once it is done with startup sync. It remains in this mode indefinitely. At the beginning of this mode, transmission is enabled. After that the node enters a sleep mode until it receives a packet, detects the end of the window, or begins a transmission. First, what happens when a packet is received, is explained. As soon as a packet is received, the MSP430 gets an interrupt from the CC2500 transceiver which pulls it out of sleep mode, and interrupt service routine "f_RxData_ISR()" is executed. This function collects information from the received packet and goes back to sleep. At the beginning of the interrupt service routine the arrival time of the packet is captured. This 
timestamp is used to find the error in clock counts. Now the received packet is uploaded from the CC2500 via SPI communication. The packet received has the format shown in figure 8.

\begin{tabular}{|c|c|}
\hline $\mathrm{rx}[0]$ & Broadcast address (0xFF) \\
\hline $\mathrm{rx}[1]$ & Transmitting Node address \\
\hline $\mathrm{rx}[2]$ & Upstream data (Node address + Sensor data) \\
\hline $\mathrm{rx}[3]$ & Upstream data (Error data) \\
\hline $\mathrm{rx}[4]$ & Downstream data (Node address + Sensor data) \\
\hline $\mathrm{rx}[5]$ & Downstream data(Error data) \\
\hline
\end{tabular}

Figure 8. Table showing the received packet structure.

Once a packet is received, two things are done. First, the error in the packet arrival time with respect to the expected nominal arrival time is determined. This gives the clock offset with respect to our current clock count. This is stored in a buffer (error_buff), and once all the errors from our neighboring nodes are collected. This buffers information can be used to make clock count corrections at the end of the window. This will be explained later. A sample of how the error is calculated is shown below.

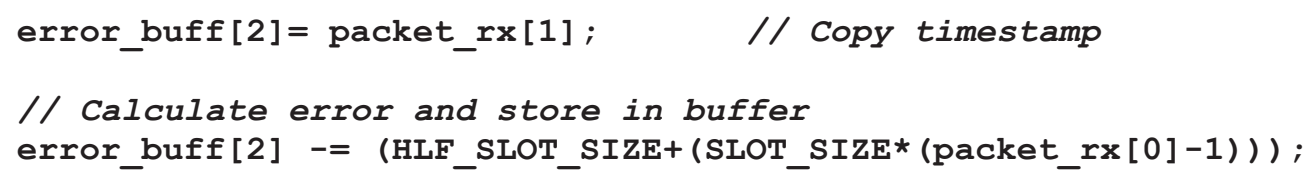

The second thing that has to be done is to store the upstream and the downstream data in a queue so that they can be forwarded during this node's transmit slot. The queue consists of three sub queues - the current queue carrying data to be transmitted during the current time slot, the next queue carrying data to be transmitted during the next time slot and the future queue carrying data to be transmitted during the time slot after the next time slot. Once the queue is updated and the error is calculated and stored in the error buffer, the node can go back to sleep until the next interrupt. This procedure is repeated every time a packet is received. The process of storing data in the queues as they are received is described next. The flow chart can be found in Appendix A (flow chart 2).

The shifting between queues can be better explained using the figures 9-12. Data received from the node's immediate neighbors (node address \pm 1 ) are stored in the next queue as shown in figures 9 and figure 11, this will be transmitted during this node's time slot in the next window. Similarly, data received from the node's second immediate neighbors (node 
address \pm 2 ) are stored in the future queue as shown in figures 10 and figure 12, this will be transmitted during this node's time slot in the window after the next window.

If data received is from the node above (node address +1 ), the downstream data is copied to the queue as shown in figure 9.

\begin{tabular}{|c|c|c|c|c|}
\hline Index & {$[0]$} & {$[1]$} & {$[2]$} & [3] \\
\hline Current queue & & & & \\
\hline Next queue & & & Downstream data [0] & Downstream data [1] \\
\hline Future queue & & & & \\
\hline
\end{tabular}

Figure 9. Data stored in the next queue.

If data received is from the node above that (node address+2), the downstream data is copied to the queue as shown in figure 10.

\begin{tabular}{|c|c|c|c|c|}
\hline Index & {$[0]$} & {$[1]$} & {$[2]$} & [3] \\
\hline Current queue & & & & \\
\hline Next queue & & & & \\
\hline Future queue & & & Downstream data [0] & Downstream data [1] \\
\hline
\end{tabular}

Figure 10. Data stored in the future queue.

If data received is from the node below (node address-1), the upstream data is copied to the queue as shown in figure 11.

\begin{tabular}{|c|c|c|c|c|}
\hline Index & {$[0]$} & {$[1]$} & [2] & [3] \\
\hline Current queue & & & & \\
\hline Next queue & Upstream data [0] & Upstream data [1] & & \\
\hline Future queue & & & & \\
\hline
\end{tabular}

Figure 11. Data stored in the next queue.

If data received is from the node below that (node address-2), the upstream data is copied to the queue as shown in figure 12.

\begin{tabular}{|c|c|c|c|c|}
\hline Index & {$[0]$} & [1] & [2] & [3] \\
\hline Current queue & & & & \\
\hline Next queue & & & & \\
\hline Future queue & Upstream data [0] & Upstream data [1] & & \\
\hline
\end{tabular}

Figure 12. Data stored in the future queue.

The queue indices are updated at the end of every window during normal mode. The current queue index is redirected to the next queue, the next queue index is redirected to the 
future queue and the future queue index is redirected to the current queue like a loop. This is done with the code shown below.

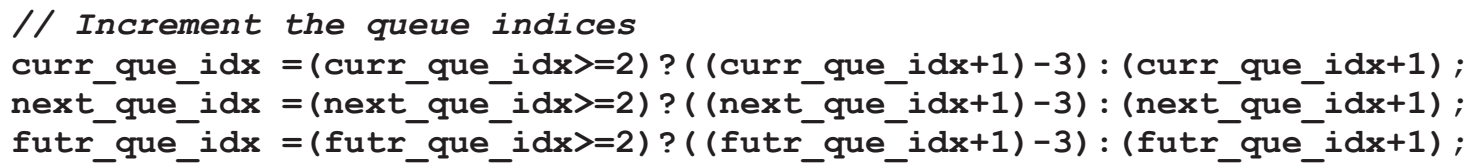

What happens every time the transmissions interrupt arrives is described next. This interrupt is triggered every time at the middle of the nodes time slot. So every node will transmit its data during this interrupt. The data packet transmitted has the structure shown in figure 13.

\begin{tabular}{|l|c|}
\hline packet_tx[0] & Packet length not including the length field \\
\hline packet_tx[1] & Broadcast address (0xFF) \\
\hline packet_tx[2] & Node address \\
\hline packet_tx[3] & Current queue[0] \\
\hline packet_tx[4] & Current queue[1] \\
\hline packet_tx[5] & Current queue[2] \\
\hline packet_tx[6] & Current queue[3] \\
\hline
\end{tabular}

Figure 13. Table showing the transmitted packet structure.

The packet shown in figure 13 is transmitted during the node's time slot. The current queue has the upstream and the downstream data that has to be relayed on to the neighboring nodes. The name of the array holding the packet is "packet_tx[]".

The data structure of the payload for both upstream and downstream is shown in figure 14. The first bit is used to send the 1-bit sensor data (motion detected or not), the next 7 bits are used for the node address and the next 8 bits (1-bit used for sign and 7-bits used for magnitude of node's clock offset) are used to send the node's current clock offset. The error is saturated to \pm 127 before transmitting.

\begin{tabular}{|c|c|c|c|c|}
\hline Bit position & $\mathbf{1 5}$ & $\mathbf{1 4 - 8}$ & $\mathbf{7}$ & $\mathbf{6 - 0}$ \\
\hline Contents & Sensor data & Node address & $\begin{array}{c}\text { Error } \\
\text { sign }\end{array}$ & $\begin{array}{c}\text { Current } \\
\text { error }\end{array}$ \\
\hline
\end{tabular}

Figure 14. Payload Data in upstream and downstream.

The node transmits its own data when the window count is 0 and just relays data received from the neighboring nodes for the rest of the windows. The code for this is shown below. The flow chart can be found in Appendix A (flow chart 1). 


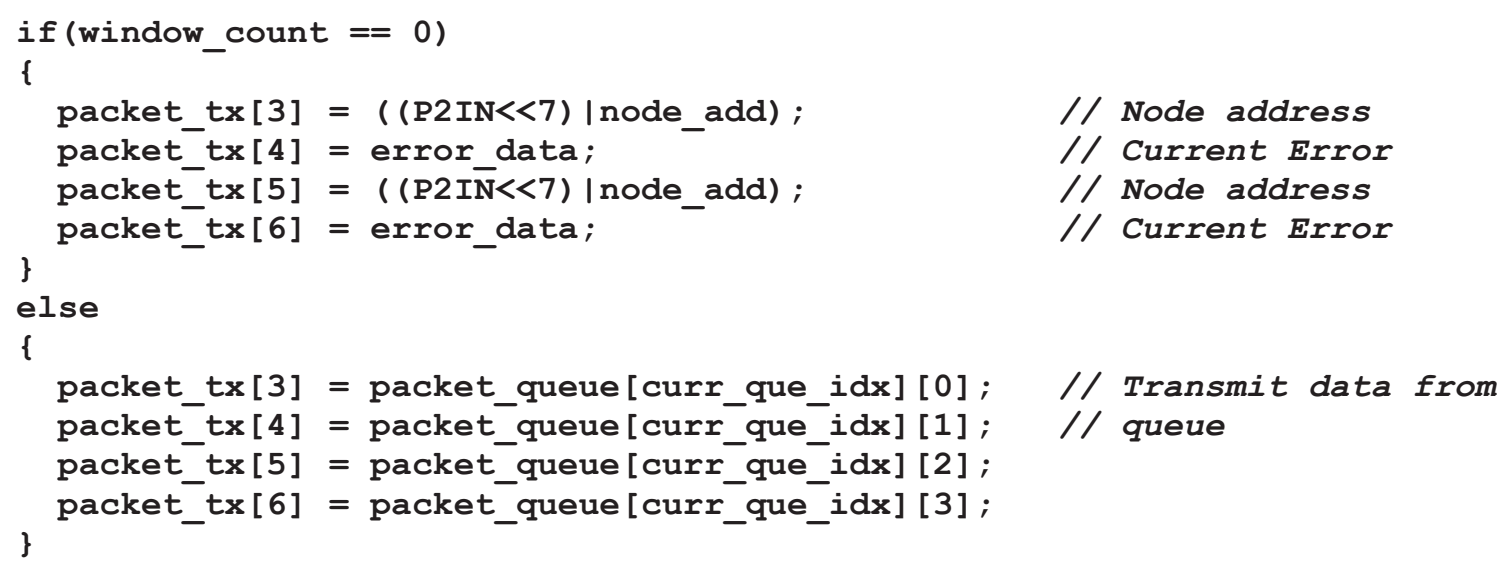

Once the data is transmitted the current queue's values are reset and the node goes back to sleep mode until the next interrupt.

The third type of interrupt happens at the end of every window. At the end of the window, the node is interrupted from sleep mode so that it can make corrections to the clock counts. This keeps the nodes synchronized to each other. All this is performed in the normal mode function "normal_mode()".

The average error is determined by picking up the minimum error from either side of the node's neighbors. The two minimum errors are averaged and the average of those is sent over to the error correction function "correct_error()". The code for this is shown below. The minimum error is determined using the min error function "min_error()".

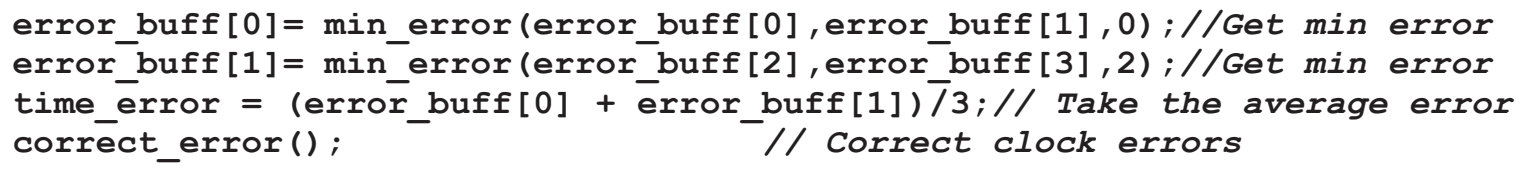

The min error function has three input parameters - two errors from the error buffer and the index. This function then returns the minimum among the two errors passed to it. Each node first sends the errors of the nodes below its node address and then sends the errors of nodes above its node address. This gives two errors, one above and one below the node address. The node then takes the average of these by summing them and dividing the sum by 3 (we are dividing by 3 to include the current node, whose error will be zero).

The min error function's code is shown below. The errors are first checked if they are negative. If they are negative the absolute value is taken. Then the minimum error among the 
two absolute values is returned. If both the errors are 0xFFF (the default value) that means no packets were received, the function will just return zero.

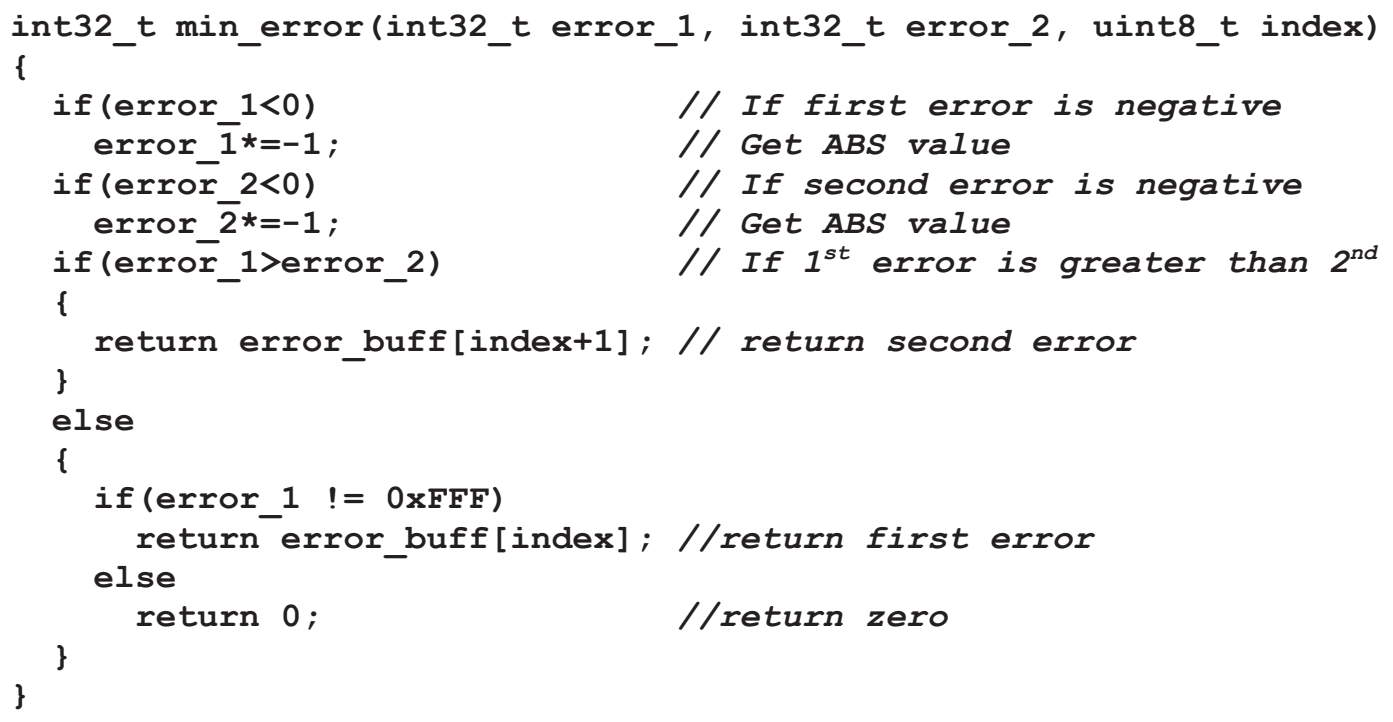

Now that the average error is calculated, it will be sent to the error correction function "correct_error()". This error will be used to correct the clock counts. The error correction code is shown below. In the error correction function, the window size is updated every $16^{\text {th }}$ window (the window_count resets every $16^{\text {th }}$ window). This is done by adding the error to the nominal window size, and the slot size is updated, the half slot size and the interrupt trigger values to reflect the changes made to the window size. Since the window correction is done only once every $16^{\text {th }}$ window the node will just correct the TAR (counter value) during the remaining time. At the end of the error correction function, the error buffer values are reset to 0xFFF. The flow chart can be found in Appendix A (flow chart 3).

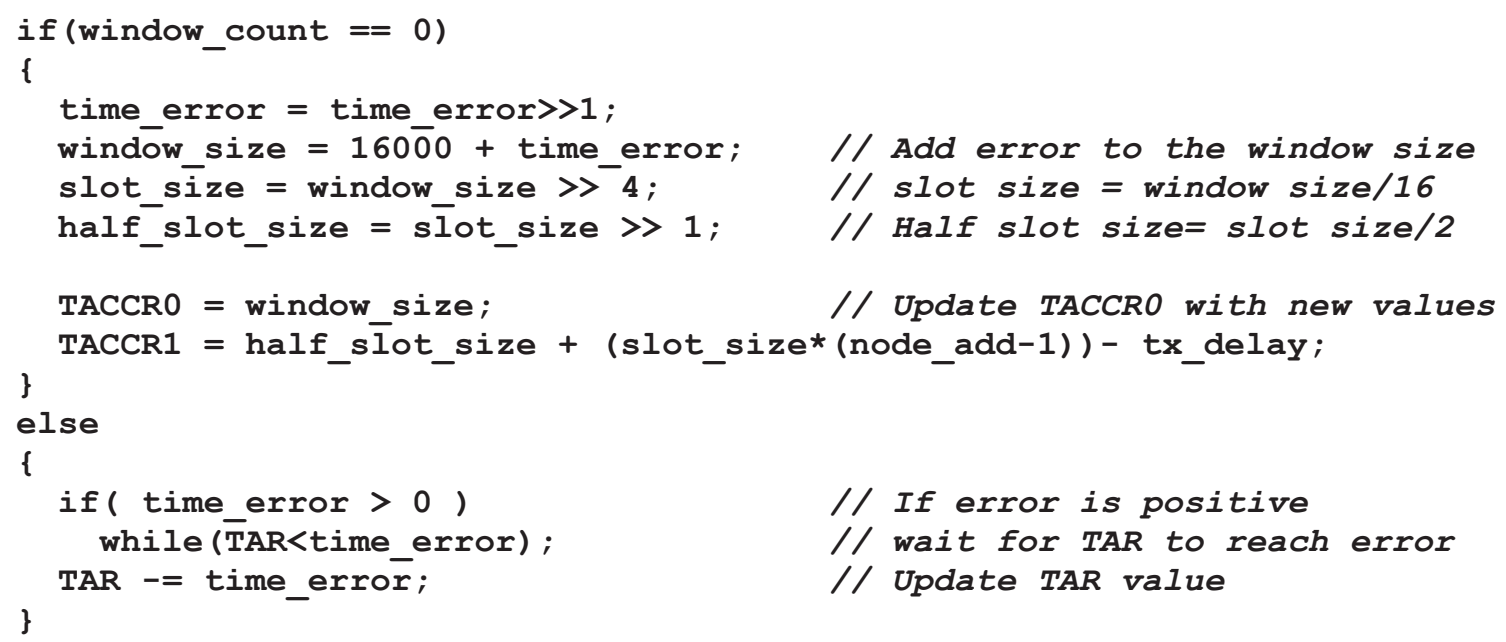



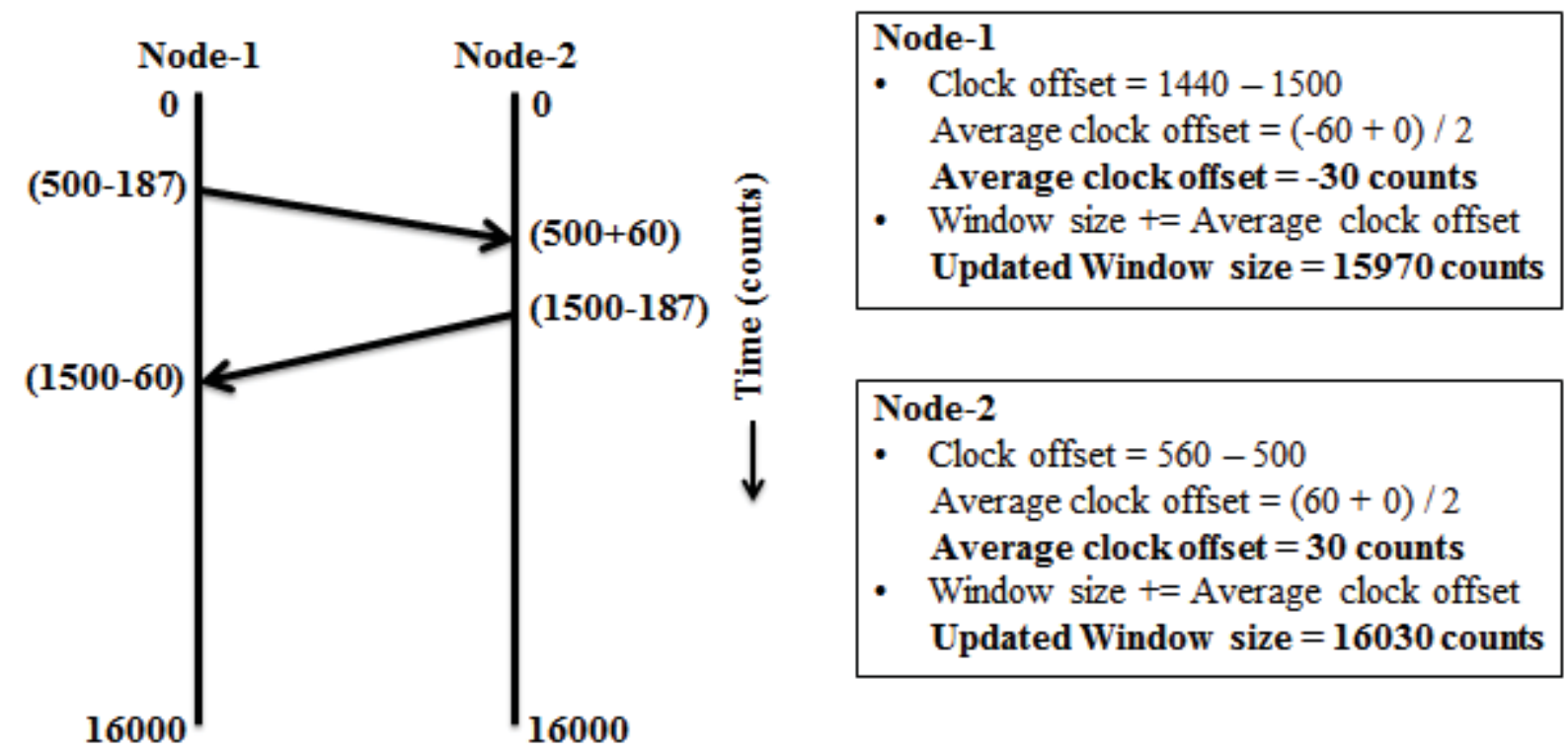

Figure 15. Clock corrections between 2 nodes.

For example, figure 15 shows clock correction procedure for 2 nodes. In this example, node-2's clock is 60 counts faster than node-1's clock. Which means when node-1's clock reaches 16000 counts, node-2's clock would have rolled over and reached 60 counts. The total transmission time is 187 counts. Each node transmits ahead of time, so that the receiver receives it at the expected arrival time. So node- 1 would transmit its packet when its counter reaches (500-187) counts, this would arrive at node-2 at (500+60) counts. Similarly, node-2's packet would arrive at node-1 at (1500-60) counts. Then both the nodes calculate the clock offset as shown in the figure 15. the average clock offset is then added to the window size. The node's then count towards the updated window size in the next window.

\subsection{Receiver node software implementation}

This section describes in detail the receiver node's software development and the software application used to monitor the nodes' activity. The receiver node's CC2500 is also configured to generate an interrupt every time a packet is received successfully. Once the packet is received, the receiver node compares the node address of the received packet with the target node address. If the received node address matches the target node address, the rest of the packet is processed to be sent over the UART and to the USB debugger. The eZ430's USB debugger is hard coded by the manufacturer to send data at 9600 bauds [5]. Due to this limitation either upstream or downstream data can be sent over the UART, but not both. 
The data to be sent over UART consists of the node's address, its sensor status and the node's corresponding clock count offset with respect to the nominal. The node address and the sensor status are masked before they are sent over UART because some of the node addresses correspond to control commands in the UART. To avoid this error, the empty bit fields are masked before transmitting. Similarly the node's corresponding error is a random value which could also match some of the control commands in the UART; hence it is split into 2 bytes (upper and lower nibble) and masked before transmitting. At the end of transmission, a new one word line character " $\mathrm{n}$ " is transmitted. This is transmitted to indicate the end of transmission and also acts as a reference to separate the data packets at the computer's end. The stream flag is used to switch between upstream and downstream. The code snippet is shown below.

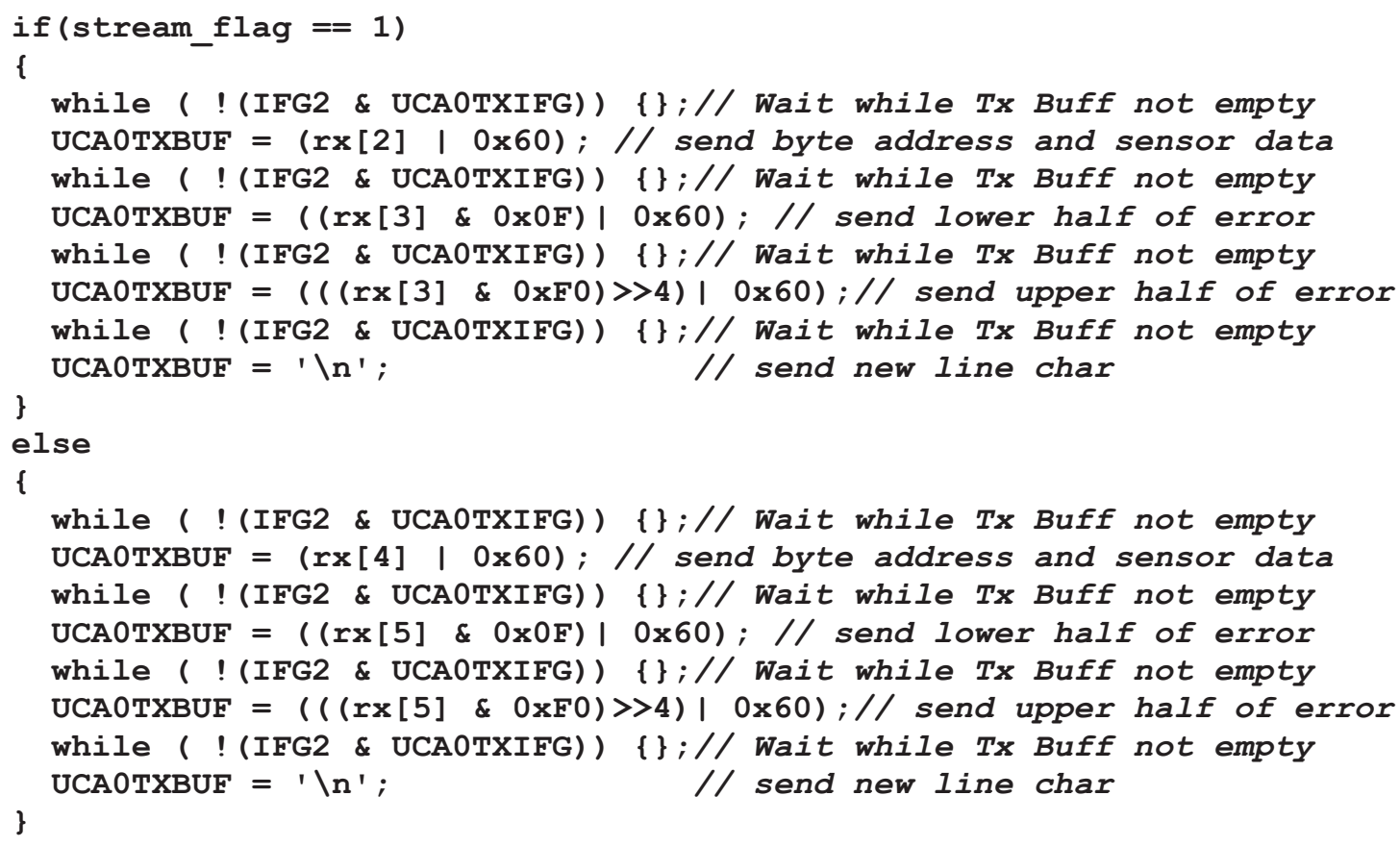

\subsection{Software development of the monitoring GUI}

This section gives an overview of the software used to monitor each node's activity on a host computer. The tool helps monitor the sensor data and the error in each node. This provides real time information of each node's activity. This tool is designed to monitor data from all sixteen nodes. The tool can be used to tap into any target node and collect data. This is possible as the nodes relay data in both directions. This provides the flexibility to collect 
data from the first node, the last node or any node in-between. The tool was designed and programed using C\#. The tool is designed to be used in tandem with a receiver node (code described in the section 6.2). The receiver node is programed to collect data from a target node; the collected data is then sent over to the tool in the computer via UART communication. The tool also sends instructions back to the receiver node. The instruction could be the desired target node or the upstream or downstream data from the desired target node. Further details on how to use the tool can be found in the user manual (Appendix B). 


\section{Testing and validation}

This section validates the working of the prototype. To test the protocol, sixteen nodes were built. All sixteen nodes were laid out as shown in figure 1. The nodes were monitored from node 1's upstream data through the receiver node. The nodes stay synchronized for extended period of time, despite significant differences in clock frequencies. The results of the experiments conducted are described in this section.

\subsection{Steady state output for all 16 nodes}

The steady state output of all 16 nodes was monitored for a few minutes and the sync offset for each node with respect to nominal clock counts was buffered for the entire duration. 269 samples were accumulated, then the minimum, maximum and the average offset was calculated for each node and results were plotted as shown in figure 16.

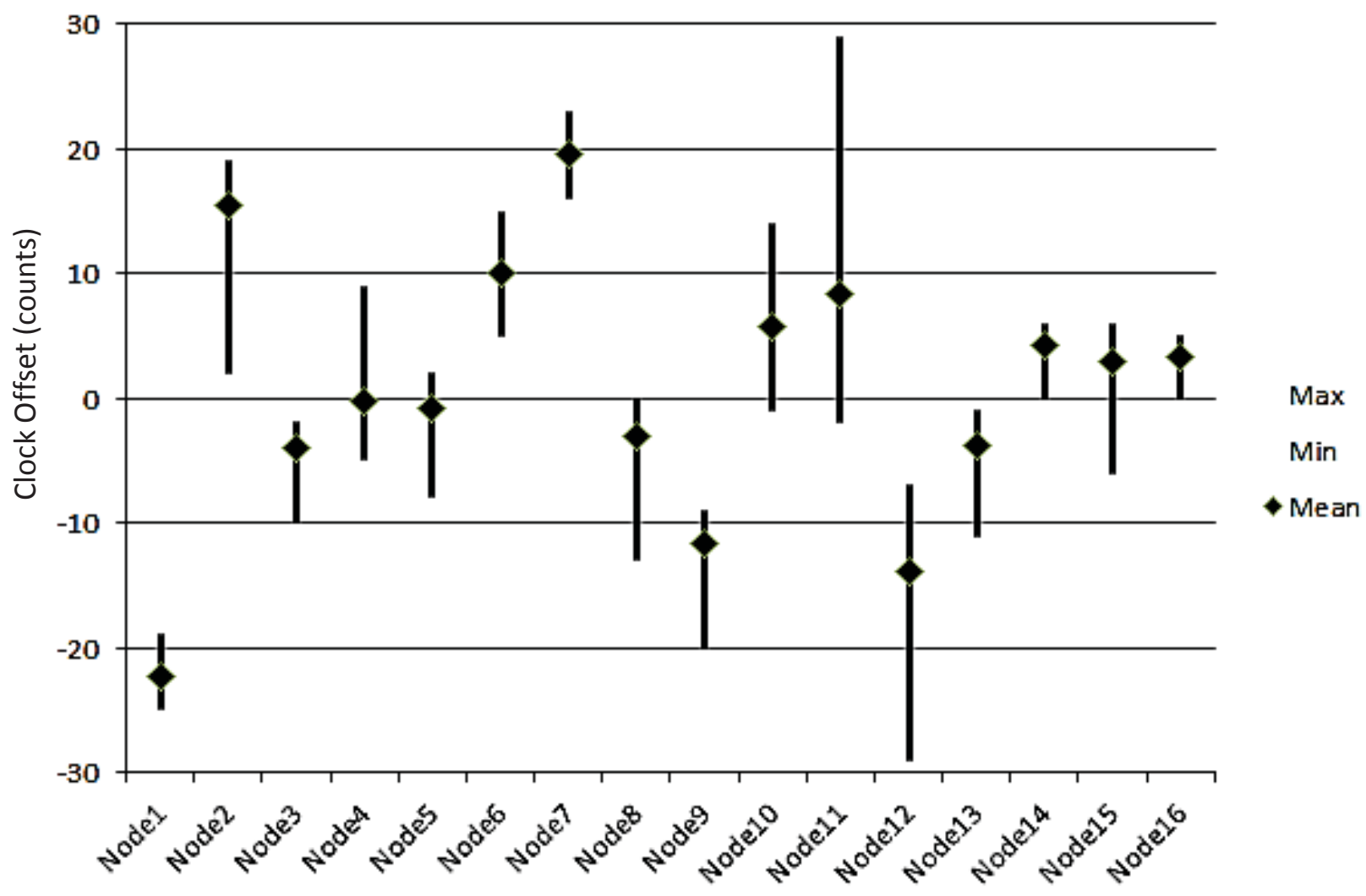

Figure 16. Minimum, maximum and average sync offsets for all 16 nodes. 


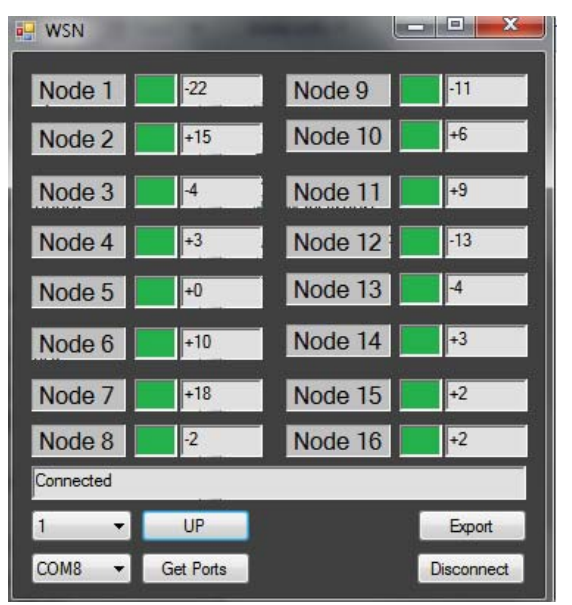

\begin{tabular}{|l|r|r|r|} 
& Max & Min & \multicolumn{1}{l}{ Mean } \\
\hline Node1 & -19 & -25 & -22.3454 \\
\hline Node2 & 19 & 2 & 15.46429 \\
\hline Node3 & -2 & -10 & -4.03553 \\
\hline Node4 & 9 & -5 & -0.33505 \\
\hline Node5 & 2 & -8 & -0.83673 \\
\hline Node6 & 15 & 5 & 10.04103 \\
\hline Node7 & 23 & 16 & 19.54124 \\
\hline Node8 & 0 & -13 & -3.02094 \\
\hline Node9 & -9 & -20 & -11.6875 \\
\hline Node10 & 14 & -1 & 5.817708 \\
\hline Node11 & 29 & -2 & 8.383838 \\
\hline Node12 & -7 & -29 & -13.8883 \\
\hline Node13 & -1 & -11 & -3.79793 \\
\hline Node14 & 6 & 0 & 4.190722 \\
\hline Node15 & 6 & -6 & 2.989637 \\
\hline Node16 & 5 & 0 & 3.289474 \\
\hline
\end{tabular}

Figure 17. GUI showing each and every node's activity (left). Where green indicates that the node is up and running. Clock offset for each node (right).

In figure 16, the x-axis is the node number and the y-axis is the sync offset with respect to the nominal in clock counts. Each clock count is equal to 4 microseconds. The zero on the $\mathrm{y}$-axis is the nominal count. Each node's minimum, maximum and the average clock count offset is shown in the graph in figure 16. It can be shown in the graph that the clock offset is bounded within a limit for each node. This clock offset is applied to the node's window size while making clock corrections. As expected, the clock offsets are non-zero as there will be constant variation from the clock source. A negative offset indicates that the node's clock is slower than its neighbors. Similarly a positive offset indicates that the node's clock is faster than its neighbors.

\subsection{Network's response with node 2 turned off}

This test was performed to demonstrate the impact of a missing node. Once the nodes reached steady state, node 2 was turned off. The table in figure 18 shows node 2's four neighbors offsets when node 2 was turned off and then turned back on. Node 1 and node 3 are dependent on node 2 for their clock synchronization; this can be seen in figure 18. As soon as node 2 is turned off, the magnitude of node 1's offset decreases and node 3's offset increases. Now both node 1 and node 3 are synchronizing with each other.

\begin{tabular}{|c|c|c|c|c|c|c|c|c|c|c|c|c|c|}
\hline Node1 & -19 & -19 & -19 & -19 & -15 & -14 & -16 & -14 & -14 & -16 & -22 & -19 & -19 \\
\hline Node2 & 17 & 18 & 18 & & & & & & & & 26 & 15 & 19 \\
\hline Node3 & -1 & -1 & -1 & -1 & 2 & 4 & 3 & 4 & 4 & 3 & -2 & 0 & -1 \\
\hline Node4 & 1 & 2 & 1 & 2 & 6 & 6 & 7 & 4 & 4 & 5 & -1 & 2 & -1 \\
\hline Node5 & 3 & 1 & 2 & 2 & 5 & 5 & 6 & 6 & 6 & 7 & 0 & 2 & 3 \\
\hline
\end{tabular}

Figure 18. Table showing clock offsets for the first 5 nodes. 


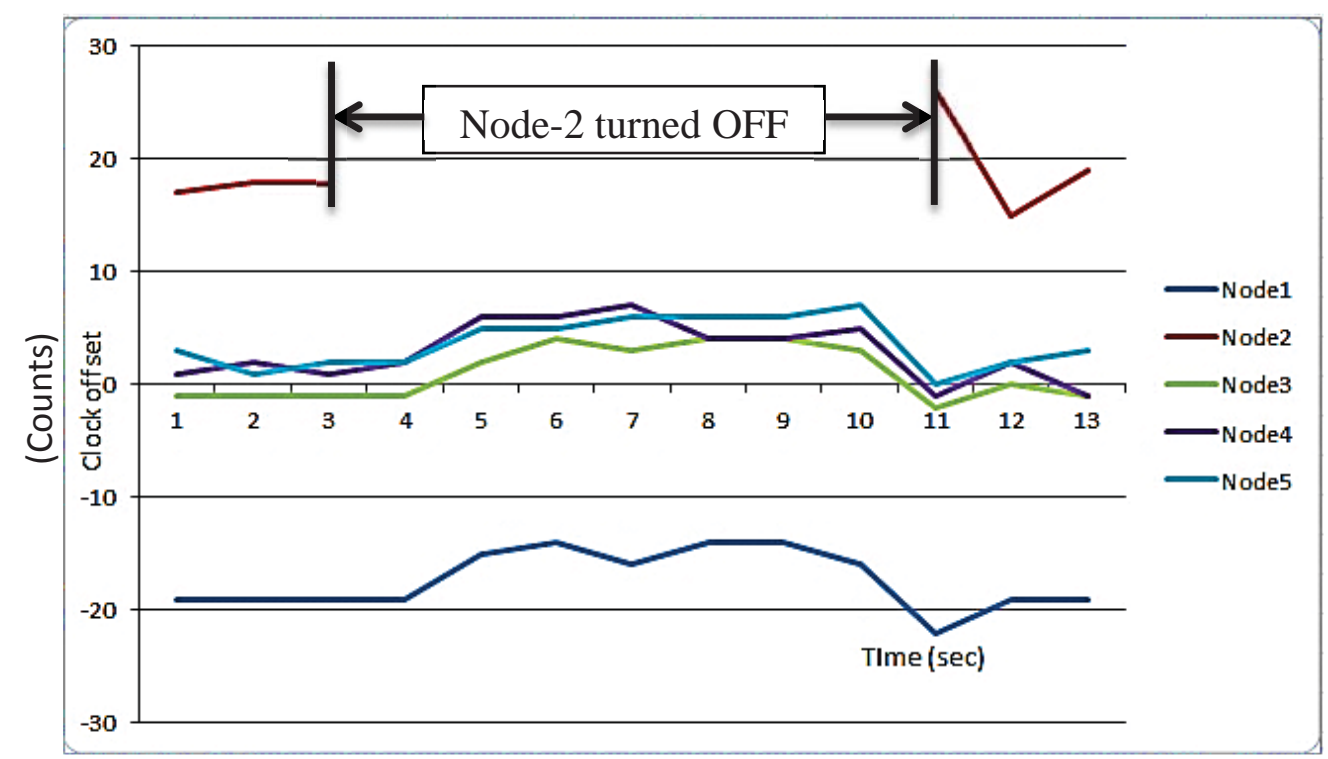

Figure 19. Graph showing the change in offsets as node 2 is turned off.

Once node 2 is turned back on, the magnitude of node 1's offset increases and node 3's offset decreases. The nodes go back to the same state as they were before. Figure 19 shows a graph of the offsets plotted from the table in figure 18. When node 2 is turned off, the offsets of node 4 and 5 also increase because node 4 is dependent on node 3 (as this is the only node to the left of node 4) and both of node 5's neighbors to the left have increased their offsets. This validates the working of the prototype for single node failure.

\subsection{Network's response with node 2 and node 4 turned off}

This test was performed to demonstrate the impact of a two alternate missing node. Once the nodes reached steady state, node 2 was turned off and after reaching steady state node 4 was turned off. The same results as the last test can be observed when node 2 was turned off (section 7.2). As soon as node 4 is turned off, both node 3's and node 5's offsets increase. This shows that node 3 and 5 were synchronized to node 4 while it was still on. The graph is shown in figure 21.

\begin{tabular}{|l|r|r|r|r|r|r|r|r|r|r|r|}
\hline Node1 & -18 & -14 & -14 & -15 & -14 & -15 & -14 & -12 & -12 & -14 & -12 \\
\hline Node2 & 17 & & & & & & & & & \\
\hline Node3 & -1 & 3 & 3 & 4 & 3 & 4 & 3 & 5 & 5 & 3 & 5 \\
\hline Node4 & 2 & 5 & 5 & 6 & 5 & 6 & 5 & & & 7 \\
\hline Node5 & 2 & 5 & 5 & 5 & 7 & 6 & 7 & 7 & 7 & 8 & 7 \\
\hline
\end{tabular}

Figure 20. Table showing clock offsets for the first 5 nodes. 


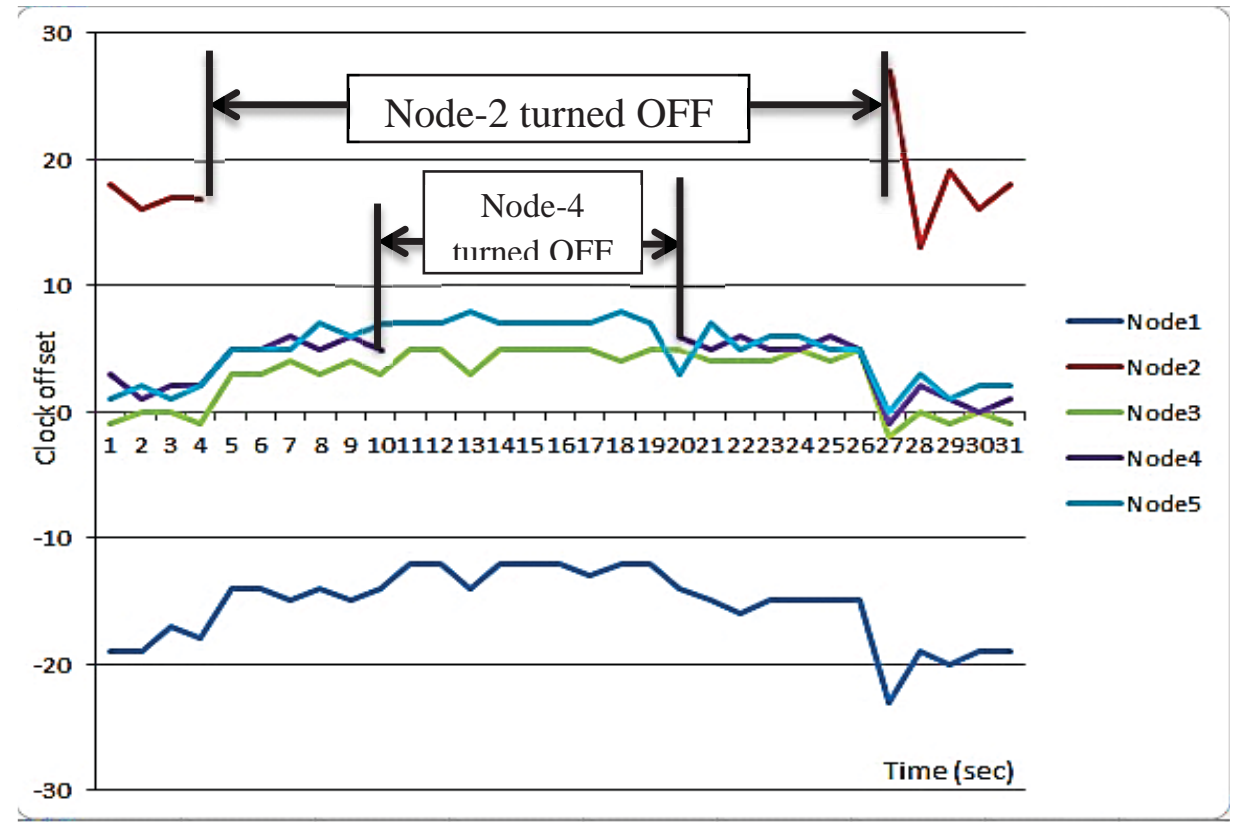

Figure 21. Graph showing the change in offsets as node 2 and 4 are turned off.

\subsection{Network's response with alternate nodes turned off}

This test was performed with all 16 nodes. To demonstrate the reliability of the network alternate nodes were turned off. Figure 22 shows the data received from node 1 when all even numbered nodes are turned off. This shows that even with single node failure, data is still routed to the end nodes. Figure 23 shows that time synchronization is still achieved even with alternate nodes off.

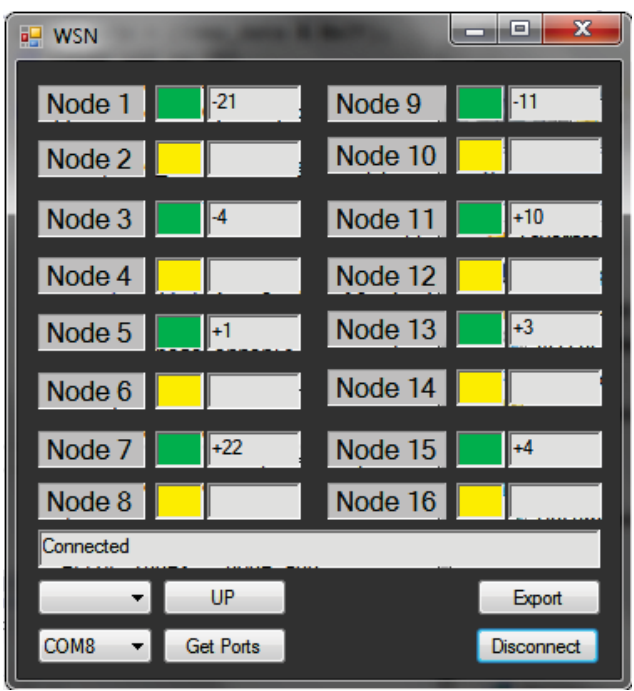

Figure 22. GUI showing the data received from node 1. 


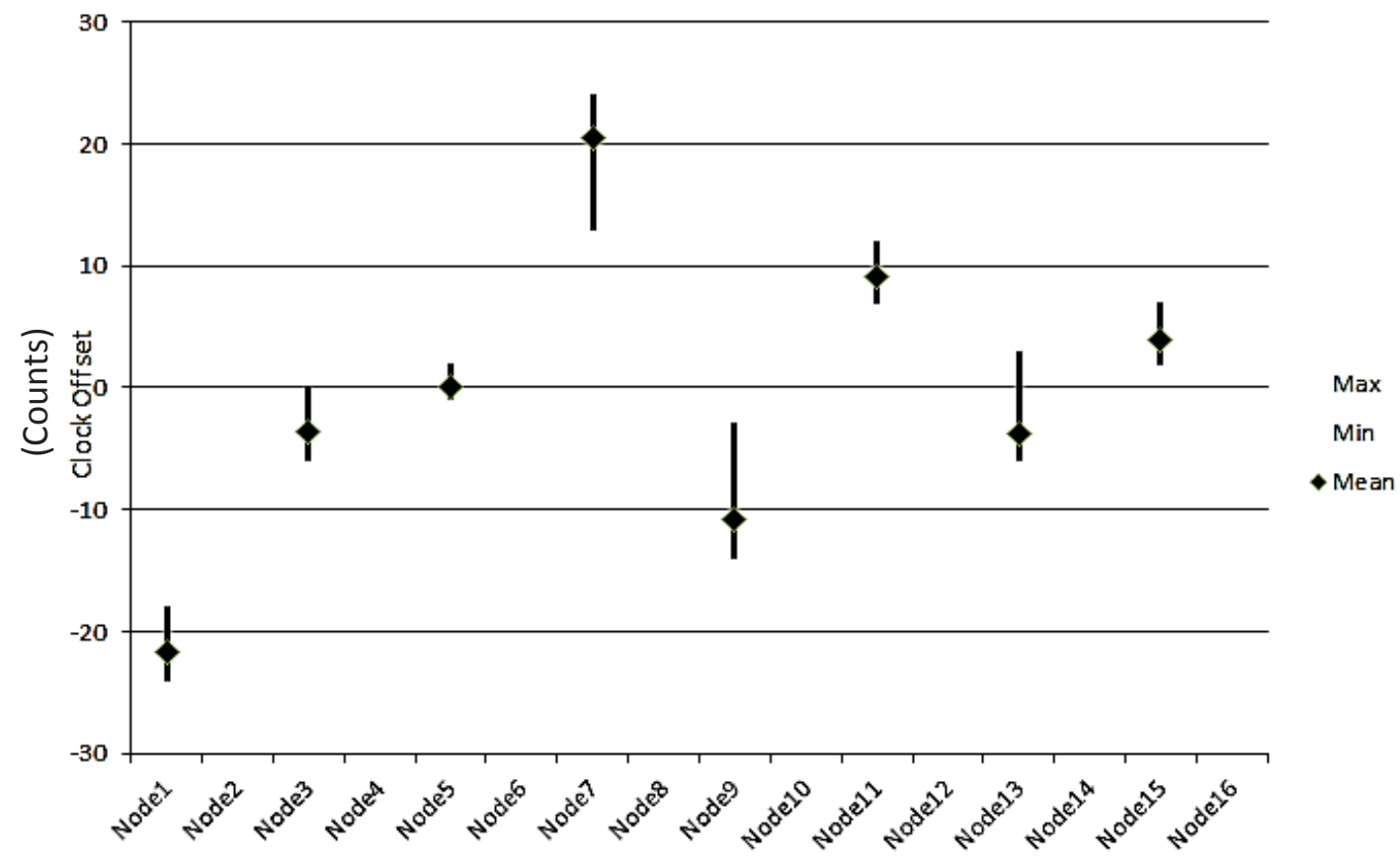

Figure 23. Min, max and average sync offsets when alternate nodes are turned off.

\subsection{Network's response with 2 adjacent nodes turned off}

This test was performed with all 16 nodes. When two adjacent nodes are turned off, this breaks the network into two halves. For example, in figure 24, nodes 6 and 7 were turned off; this breaks the link and all the data after node 6 is no longer available at node 1 . This partitions the network into 2 sub-networks (node1 to node5 and node8 to node16). The table in figure 25 shows the loss of data once both nodes 6 and 7 are turned off.

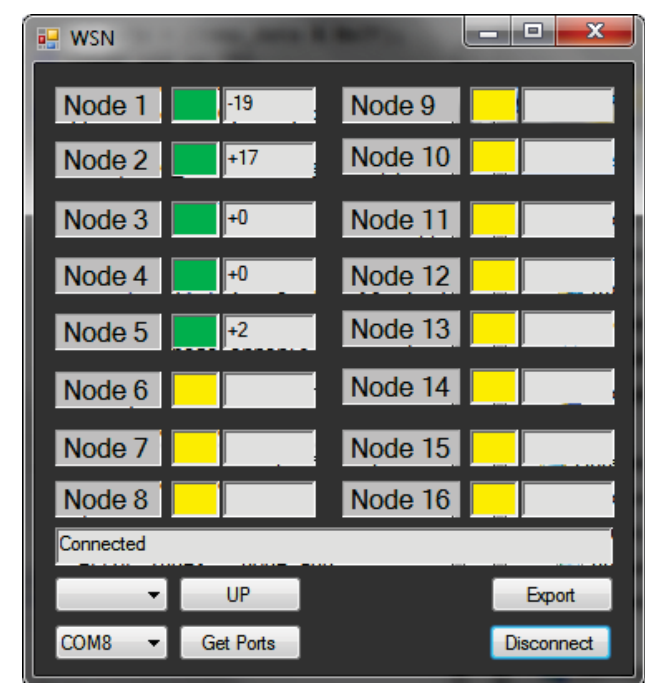

Figure 24. GUI showing the data received from node 1. 


\begin{tabular}{|c|c|c|c|c|c|c|c|c|c|c|}
\hline A & $x$ & $Y$ & Z & AA & $A B$ & $A C$ & $A D$ & $\mathrm{AE}$ & AF & AG \\
\hline Node1 & -20 & -22 & -23 & -22 & & -22 & -22 & -23 & -19 & -19 \\
\hline Node2 & 11 & 15 & 14 & 14 & 14 & 14 & 15 & 14 & 16 & 17 \\
\hline Node3 & -2 & -4 & -4 & -4 & -4 & -4 & & -1 & 0 & -2 \\
\hline Node4 & 2 & -1 & 0 & 0 & 0 & -1 & 3 & 3 & 3 & 3 \\
\hline Node5 & 0 & -1 & 0 & -1 & -1 & -1 & 2 & 2 & 2 & 2 \\
\hline Node6 & 10 & & & & & & & & & \\
\hline Node7 & 20 & 20 & 20 & 21 & 20 & 20 & & & & \\
\hline Node8 & -1 & -3 & -4 & -4 & -5 & -4 & & & & \\
\hline Node9 & -12 & -11 & -13 & -11 & -11 & -12 & & & & \\
\hline Node10 & 5 & 4 & 5 & 4 & 6 & & & & & \\
\hline Node11 & 10 & 9 & 9 & 9 & 8 & & & & & \\
\hline Node12 & -14 & -13 & -12 & -14 & -15 & & & & & \\
\hline Node13 & -5 & -6 & -5 & -4 & -6 & & & & & \\
\hline Node14 & 3 & 4 & 2 & 4 & 3 & & & & & \\
\hline Node15 & 4 & & 3 & 1 & 3 & & & & & \\
\hline Node16 & 3 & 3 & 3 & 2 & 3 & & & & & \\
\hline
\end{tabular}

Figure 25. Table showing clock offsets received from node 1.

\section{Design constrains and compromises}

Now that the system has been described in its entirety, this section discusses in detail some constrains faced while designing the prototype.

\subsection{Design considerations for production unit}

The prototype was built to demonstrate that the concept can be used to provide a reliable warning system for pedestrians. The prototype used PIR motion sensors to detect the presence of an object, but can be replaced with a more sophisticated detection method to detect the presence of a train, while minimizing false detections. The prototype uses LED's to notify the pedestrians, this can also be replaced with other warning systems such as lights and horns. The prototype was designed to forward alert to \pm 1 node; this can be extended to $\pm \mathrm{N}$ nodes. The prototype software was designed to accommodate sixteen nodes; this can also be further extended by reusing the time slots.

The initial design of the prototype was to make the nodes pick their addresses automatically. The nodes at startup would listen to their neighbors and based on that would pick an empty time slot and an address corresponding to the time slot. This can be implemented in the production nodes because in the field the nodes can clearly hear only their neighbors. Thus they can detect empty time slots and pick those time slots. In the 
prototype, a starting node could hear several other nodes due to the short distance involved. Thus, the nodes were hardcoded with their addresses and pick the time slots corresponding to their addresses.

\subsection{Other design constrains}

The receiver node uses a UART to communicate with the user's computer. The eZ430RF2500's USB debugger was used to communicate with the computer. Unfortunately, the UART communication rate is fixed to 9600 baud [5]. This resulted in limiting the maximum number of bytes that could be transmitted in one time slot. At the rate of 9600 bauds it will take 3.3 msec to transmit 4 bytes over UART and the time slot for each node was 4 msec. To send both the upstream and the downstream data, 8 bytes had to be transmitted (it takes 6.6 msec to send 8 bytes). Given the fact that only 4 bytes can be sent within each time slot the GUI is limited to displaying only the upstream or the downstream data. This limitation is only when the user tries to receive data from nodes other than 1 and 16 . All the nodes can be monitored from the end nodes. For node 1, only the downstream is sufficient and for node 16 only the upstream is sufficient.

Each node's data consists of the node address, sensor status and the node's clock offset. The node address and the sensor status can be packed into one byte but the clock offset had to be split into two bytes. The node's clock offset is a random number and because of that it would sometimes match the ASCII control codes, resulting in undesirable output at the receiver end. To avoid this issue the offset is split into 2 parts (upper nibble and the lower nibble). This is then packed into 2 bytes and masked before transmitting. At the end of each node's data a delimiter (new-line character) has to be sent; this is sent to help the receiver separate the node's data. All this put together is 4 bytes in length.

\subsection{Choosing the proper frequency for communication}

The CC2500 uses the $2.4 \mathrm{GHz}$ band which is commonly used by many devices, including Wi-Fi routers. Routers commonly use channels 1 (2.401-2.423 GHz), 6 (2.426-2.448 GHz) and $11(2.451-2.473 \mathrm{GHz})$ [7] as shown in figure 26. But some routers use channels other than the ones mentioned above. The frequency for the prototype was chosen experimentally to start at $2.4508 \mathrm{GHz}$. This is in between Wi-Fi channels 6 and 11 . Due to the fact that the 
channel could still be busy, carrier sense had to be disabled because this would delay the transmission, which is not acceptable in a time critical application.

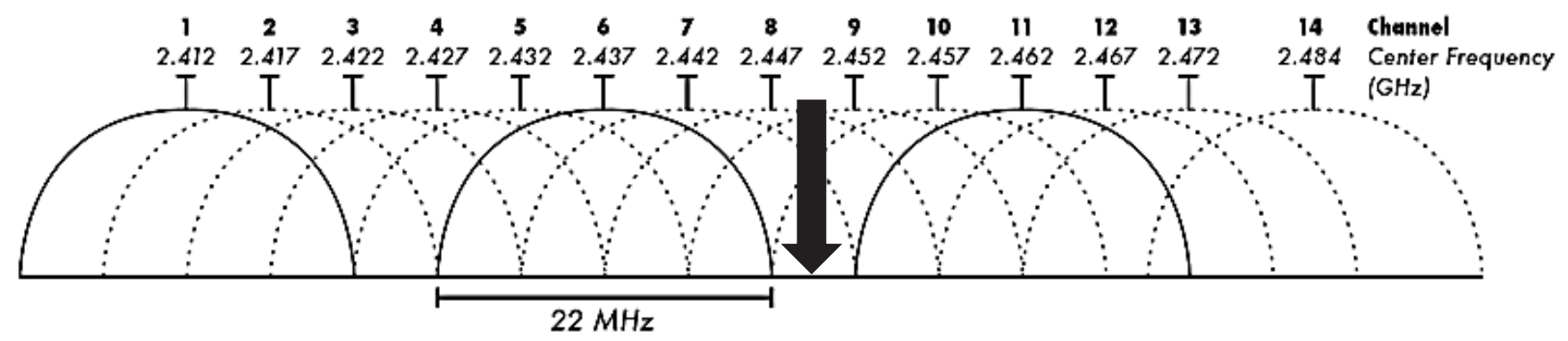

Figure 26. Wi-Fi Channel spectrum [6].

\subsection{Total packet transmission time}

The protocol relies on TDMA which makes time synchronization critical. Since the clock offset is determined based on the received packets arrival time the transmission delay has to be included. The transmission delay can be determined analytically as shown below.

\section{Total transmission time:}

$$
\mathrm{T}_{T x}=\mathrm{T}_{S P I}+\mathrm{T}_{W T x}
$$

Where:

$$
\begin{aligned}
& \mathrm{T}_{T X}=\text { Total transmission time. } \\
& \mathrm{T}_{S P I}=\text { MSP to CC2500 SPI transfer time at } 500 \mathrm{Kbps} . \\
& \mathrm{T}_{W T x}=\text { Wireless transmission time at } 250 \mathrm{Kbps} .
\end{aligned}
$$

\section{SPI transfer time:}

$$
\begin{aligned}
& \mathrm{T}_{S P I}=\mathrm{L}_{T x_{-} F I F O}+\mathrm{L}_{D}+\mathrm{L}_{T x_{-} s t b} \\
& \mathrm{~T}_{S P I}=(1 \text {-byte }+7 \text {-bytes }+1 \text {-byte }) \text { at } 500 \mathrm{Kbps} . \\
& \left.\mathrm{T}_{S P I}=\text { (9-bytes } * \text { 8-bits }\right) / 500 \mathrm{~K}=0.144 \text { msec. }
\end{aligned}
$$


Where:

$\mathrm{L}_{D}=$ Length of payload data in bytes.

$\mathrm{L}_{\text {TX_FIFO }}=$ Length of Tx FIFO register's address in bytes.

$\mathrm{L}_{T x_{-} s t b}=$ Length of transmission strobe command in bytes.

\section{Wireless transmission time:}

$$
\begin{aligned}
& \mathrm{T}_{W T x}=\mathrm{L}_{P r e}+\mathrm{L}_{S y n c}+\mathrm{L}_{D}+\mathrm{L}_{C R C} \\
& \mathrm{~T}_{W T x}=(4 \text {-bytes }+32 \text {-bits }+7 \text {-bytes }+16 \text {-bits }) \text { at } 250 \mathrm{Kbps} \\
& \mathrm{T}_{W T x}=(17 \text {-bytes } * \text { 8-bits }) / 250 \mathrm{k}=0.544 \text { msec. }
\end{aligned}
$$

Where:

$$
\begin{aligned}
& \mathrm{L}_{\text {Pre }}=\text { Length of preamble in bytes. } \\
& \mathrm{L}_{S y n c}=\text { Length of sync word in bits. } \\
& \mathrm{L}_{C R C}=\text { Length of CRC in bits. } \\
& \qquad \mathrm{T}_{T x}=\mathrm{T}_{S P I}+\mathrm{T}_{W T x} \\
& \qquad \mathrm{~T}_{T x}=(0.144+0.544)=0.688 \mathrm{msec} .
\end{aligned}
$$

The above calculation does not include the time required for the CC2500 to modulate the data, CRC calculation and the propagation time in air. Experimentally, the average total time to transmit a packet was determined to be 0.748 msec. This delay is included in the packet TX interrupt. The packet is transmitted ahead of the delay time so that it arrives at the exact time expected at the receiver. In the field, propagation delay of $5.2 \mathrm{~ms} / \mathrm{mile}$ has to be added to the total transmission time. The CC2500, when in receive mode, occasionally stops receiving. To overcome this issue, the receive strobe is retransmitted to the CC2500 at the end of every window. 


\section{Conclusion}

The prototype was successfully built and demonstrated. The algorithm used for this project was also validated by the performance of the prototype. The protocol shows reliable assurance for hard real time data communication even with node failures at random locations.

The prototype can be developed into a production module with slight modifications to warn pedestrians about oncoming trains. The production model can be easily scaled to longer distances by reusing the time slots. The network deployment can be variable. For example, in areas where accidents are prone to happen, network deployment can be denser than in areas with less human activity. In places such as railway stations, bridges and curved tracks the network deployment can be denser. A denser deployment can easily catch the pedestrian's attention. In areas where there is less or no human activity and in places where there is clear visibility of oncoming trains the network deployment can be less dense.

One of the main requirements of a railway warning system is that the warning signal reaches the pedestrian well before the train does. To ensure this, the warning signal should travel faster than the train. In the prototype, it takes 1.024 seconds for the warning signal to travel from one end to the other. So if the prototype nodes are placed 50 feet apart, the warning signal travels at a speed of $499.38 \mathrm{mph}$, which is much faster than a train's speed. The warning signals speed can be improved further by reducing the time slots width or by using faster data rates between nodes. It is estimated that the propagation speed can be atleast quadrupled with minimal changes to the system software.

The prototype is designed to forward an alert to one immediate neighbor on either side. In the production model, the alert distance (number of hops from detection) can be determined based on the speed of the detected train. Thus, the alert distance can be larger for a train with higher speed and smaller for a train with lower speed. The speed of the train can be easily calculated as we know the distance between two nodes and the time it takes to travel from one node to the next. In order to avoid false detections due to track maintenance vehicles, the nodes can be programmed to receive a wireless command from the maintenance unit to inhibit detection of that vehicle, while still retaining its ability to detect oncoming trains. 
In a 2nd order power chain, such as the proof-of-concept system, when 2 adjacent nodes fail, the network is partitioned into 2 sub-networks. The two sub-networks still relay data within themselves from one end to the other end. Once either of the failed nodes recovers, the two sub-networks can automatically recombine to restore the complete network.

If this level of redundancy proves insufficient, the system can be configured as a higher order power chain. For example, in a 3rd order chain, each node communicates with its 3 nearest neighbors on either side as shown in figure 27. This would require only minor changes to the software and that the nodes are placed such that each node is within communication range of its 3 immediate neighbors on either side.

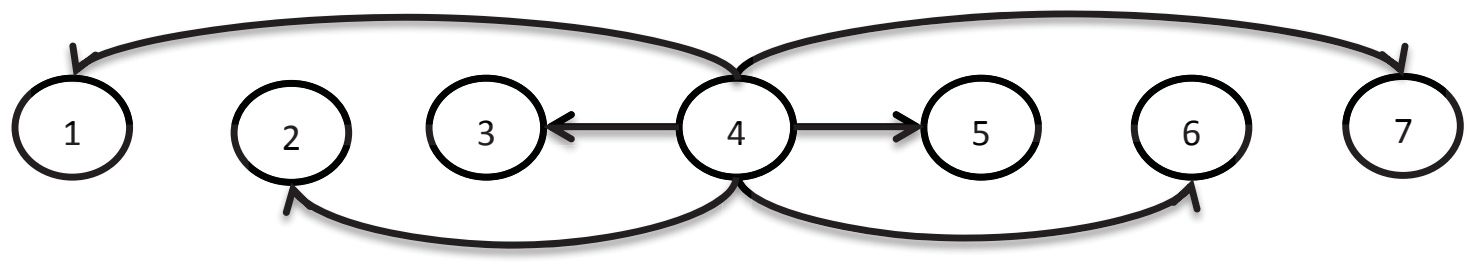

Figure 27. Node-4 communicating with 3 immediate neighbors on either side.

The total cost of each proof-of-concept unit was $\$ 58.28$. The most expensive components are the ez430-rf2500 development board (\$40) [18] and the PIR (\$9.89) motion sensor [19]. If the nodes are placed 50 feet apart from each other, there will be 105 nodes per mile. The total hardware cost would then be $\$ 6,120$ per mile. Naturally, a production system would be more costly, due to the Sensor, Annunciator, and Power Supply subsystems. Nonetheless, it is reasonable to expect the system cost to be in the range of a few tens of thousands of dollars per mile.

For future work, the nodes should be capable of predicting available node addresses at startup. Rather than hard coding the address they should be able to pick their addresses based on the information received from the neighboring nodes. It can also be used in applications where direct line of sight communication is not possible, like in tunnels, canyons and mines. 


\section{Reference}

[1] R.M. Kieckhafer, "Design of a Real-Time Wireless Network for the Northern Pierre Auger Observatory”, the Pierre Auger Collaboration, June 28, 2010.

[2] Texas instruments, eZ430-RF2500 Development Tool User guide. SLAU227A Mixed Signal Products, 2007. , Available: http://www.ti.com/lit/ug/slau227e/slau227e.pdf.

[3] Texas instruments, MSP430x22x2, MSP430x22x4 Mixed Signal microcontroller Datasheet, 2012. , Available: http://www.ti.com.cn/cn/lit/ds/symlink/msp430f2274.pdf.

[4] Texas instruments, CC2500 Low-Cost Low-Power 2.4 GHz RF Transceiver Datasheet, 2014. , Available: http://www.ti.com/lit/ds/symlink/cc2500.pdf.

[5] Texas instruments, Application Report SLAA378D, April 2011, Available: http://www.ti.com/lit/an/slaa378d/slaa378d.pdf.

[6] Wireless Medium Access Control (MAC) and Physical Layer (PHY) Specifications, IEEE Standard 802.11, 2012.

[7] Wi-Fi / WLAN Channels, Frequencies, Bands \& Bandwidths [online]. Accessed on: October 2014, Available: http://www.radio-electronics.com/info/wireless/wi-fi/80211channels-number-frequencies-bandwidth.php.

[8] Microsoft Visual Studio 2013, C\# Programming Guide. Accessed on: June 2014, Available: http://msdn.microsoft.com/en-us/library/67ef8sbd.aspx.

[9] Microsoft Developer Network, SerialPort Class. Accessed on: June 2014, Available: http://msdn.microsoft.com/en-us/library/system.io.ports.serialport\% 28v =vs.110\%29.aspx.

[10] Instructables Tutorial, Serial Port Programming With .NET. Accessed on: June 2014, Available: http://www.instructables.com/id/Serial-Port-Programming-With-NET/step1/Setup-and-Open-the-Serial-Port/

[11] St. Louise Post-dispatch, Hundreds die walking the tracks each year [online]. Accessed on: September 2014, Available: http://www.stltoday.com/news/local/metro/hundreds-diewalking-the-tracks-each-year/a rticle_b9c8bbcc-f424-559a-a0c4-bee46f8d4fe7.html

[12] The Pierre Auger Cosmic Ray Observatory [online]. Accessed on: February 2014, Available: http://www.auger.org /index.html

[13] Branislav.K, et al, "The Flooding Time Synchronization Protocol”, SenSys '04 Proceedings of the 2nd international conference on Embedded networked sensor systems, Pages 39-49, Nov 2004. 
[14] R.M. Kieckhafer, et al, "Exploiting Omissive Faults in Synchronous Approximate Agreement”, IEEE Trans. on Computers, VOL. 49, NO. 10, October 2000.

[15] Saurabh G, et al, "Timing-sync Protocol for Sensor Networks", SenSys '03 Proceedings of the 1st international conference on Embedded networked sensor systems, Pages 138 - 149, 2003.

[16] Motion sensor, PIR Sensor (\#555-28027) Datasheet, Parallax Inc., 2012.

[17] IAR Embedded Workbench, Compiler and Debugger toolchain for microcontrollers, IAR systems.

[18] Texas instruments, eZ430-RF2500 Development Tool, Available:

$<$ http://www.ti.com/tool/ez430-rf2500t\#buy>

[19] Motion sensor, PIR Sensor (\#555-28027), Parallax Inc., 2012. Available:

$<$ http://www.digikey.com/product-search/en?KeyWords=55528027> 


\section{Appendix A}

Flow chart 1: Function 'f_TxData'

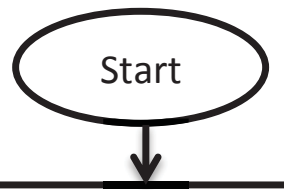

Turn on onboard LED (RED)

to indicated Transmitting

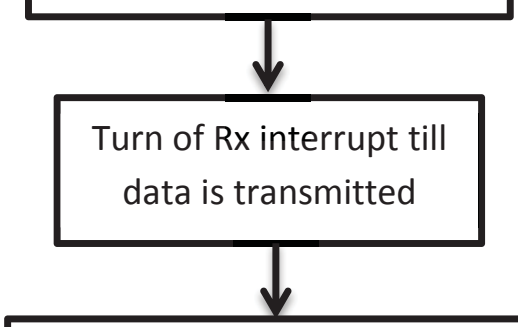

Limit the current error to \pm

127. So that it occupies only

1 byte in the payload

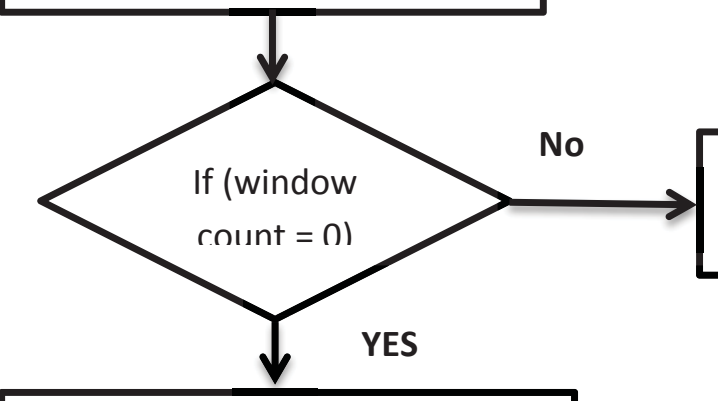

Send data

from queue

Send Node's data. This includes

the Sensor data, node address

and the current error

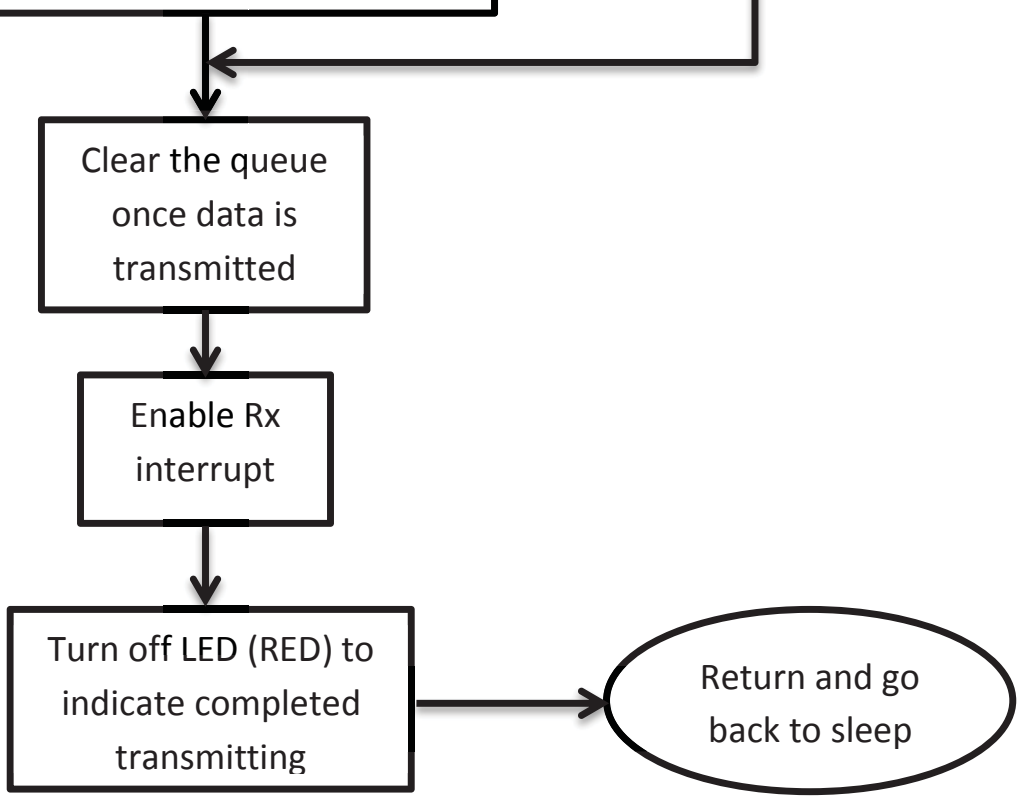


Flow Chart | 37

Flow chart 2: Function 'f_RxData_ISR'
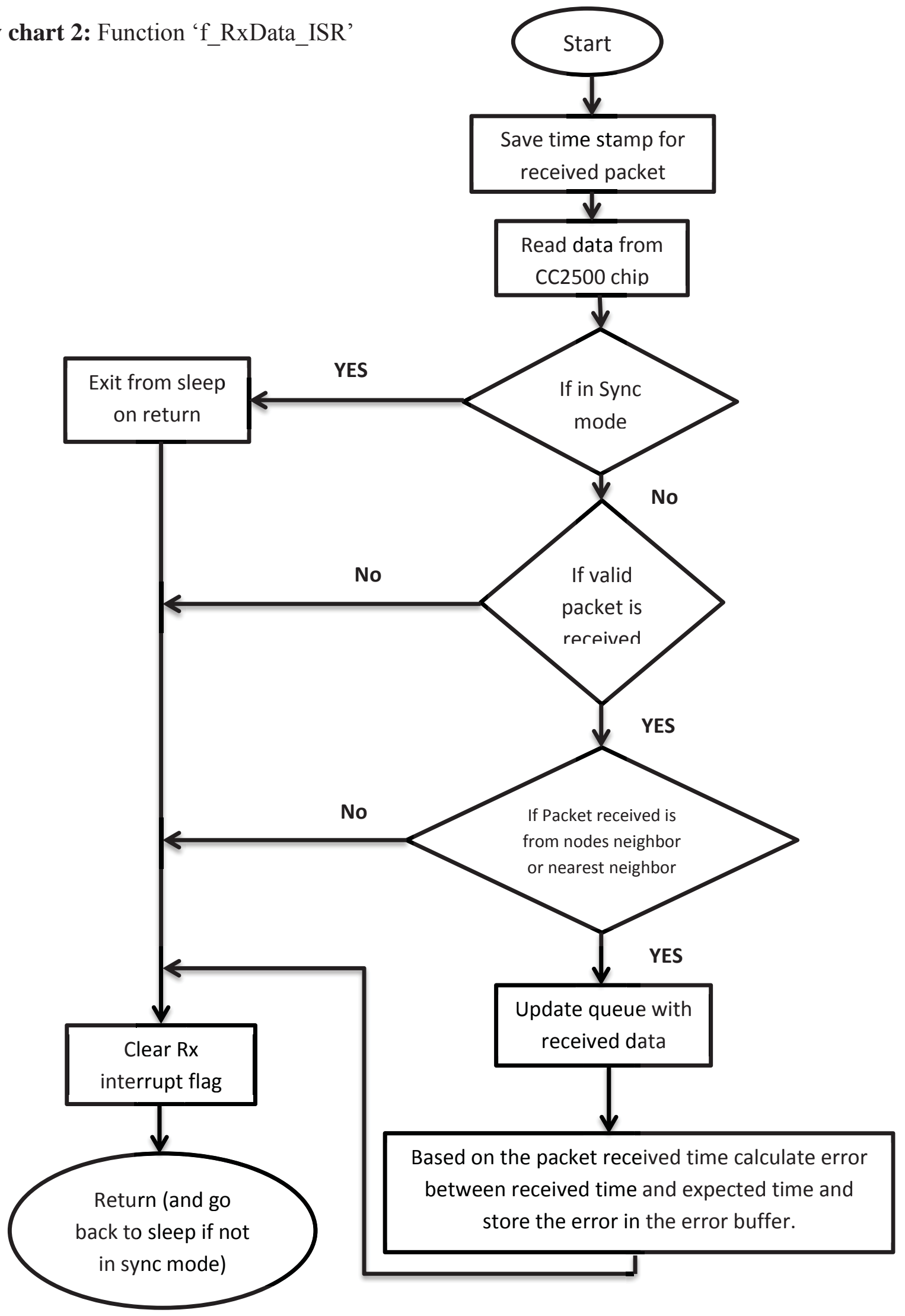
Flow Chart 38

Flow chart 3: Function 'correct_error'

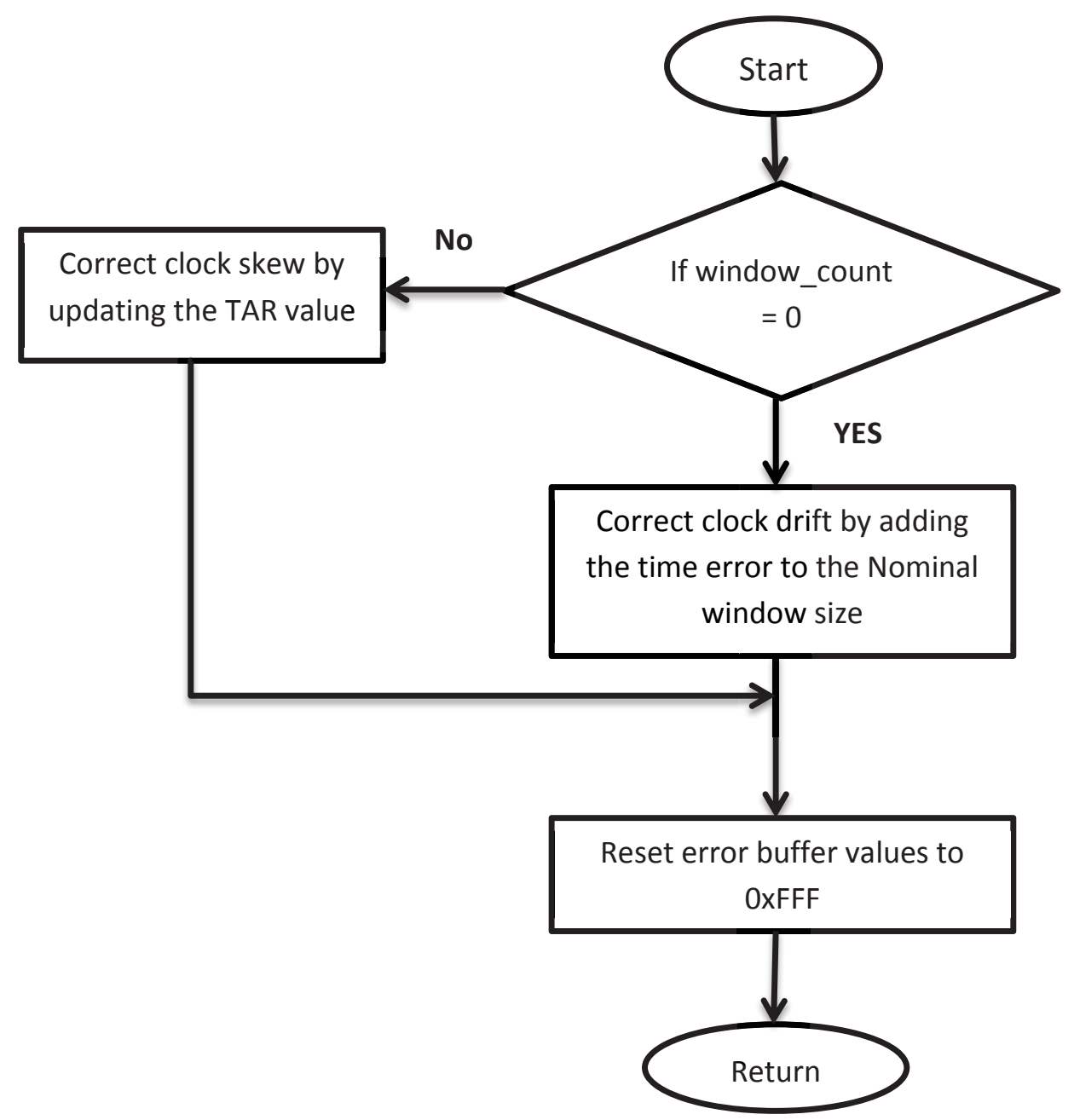


Flow chart 4: Function 'startup_sync'

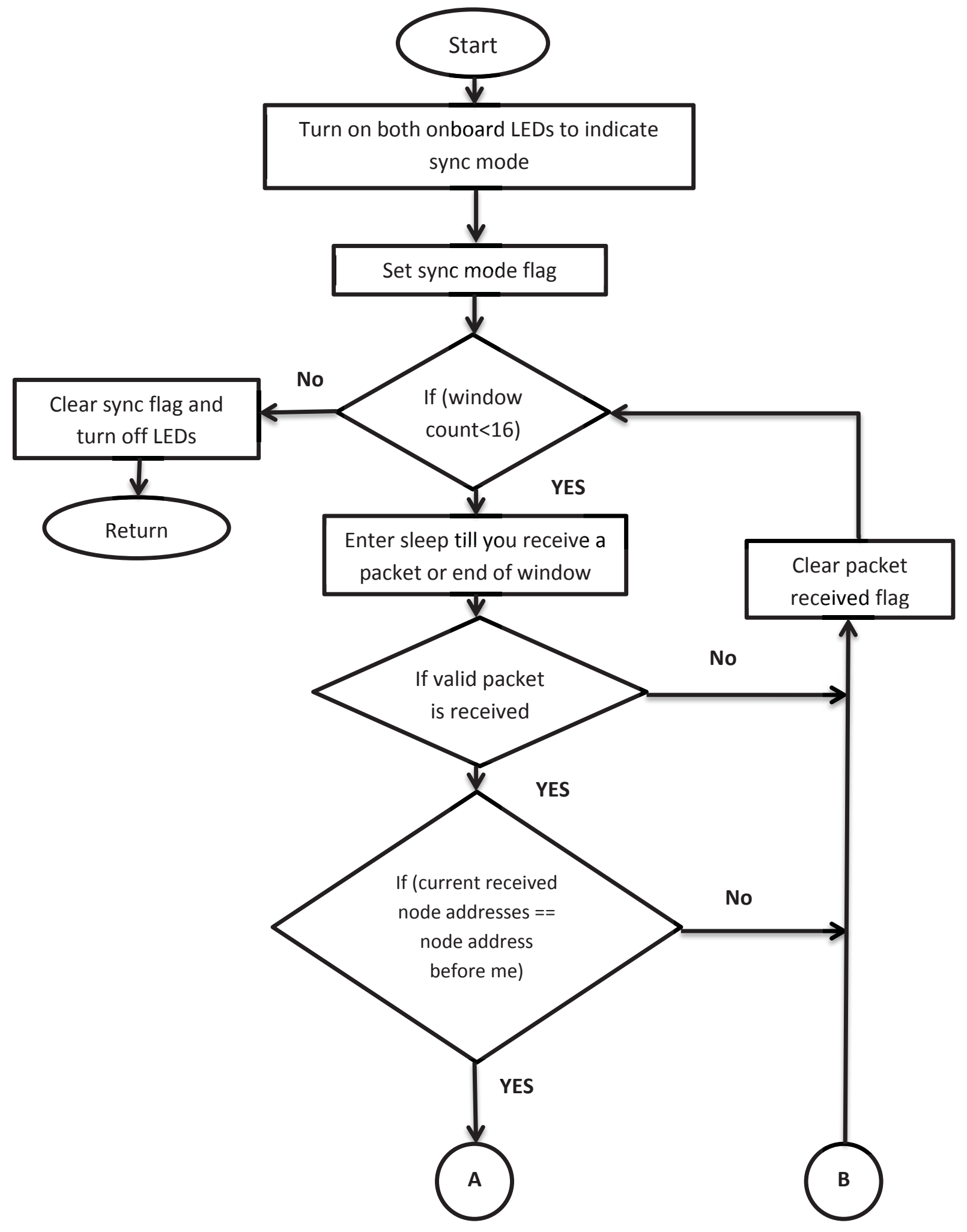


Flow Chart 40

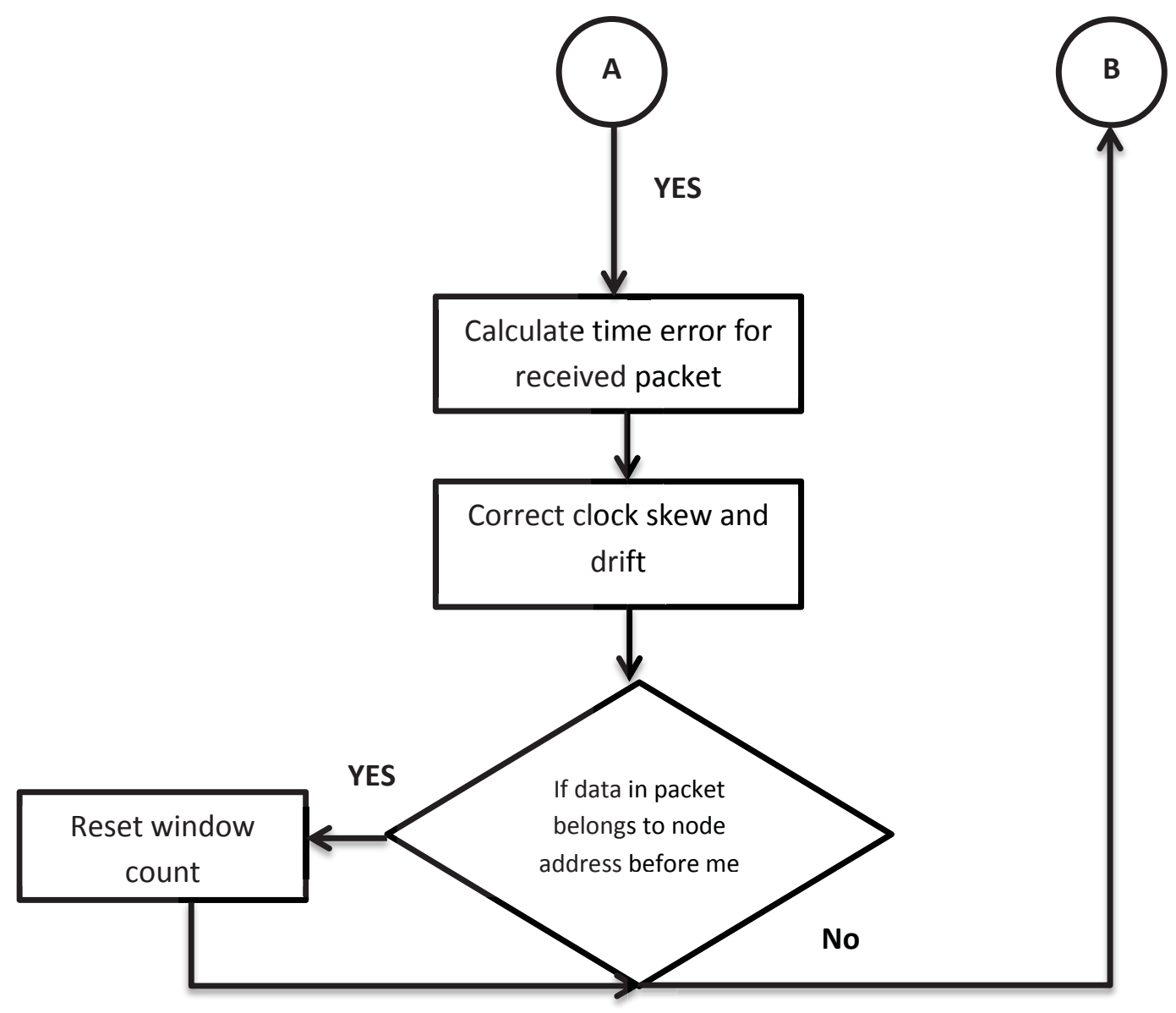


Flow chart 5: Function 'sync_check'

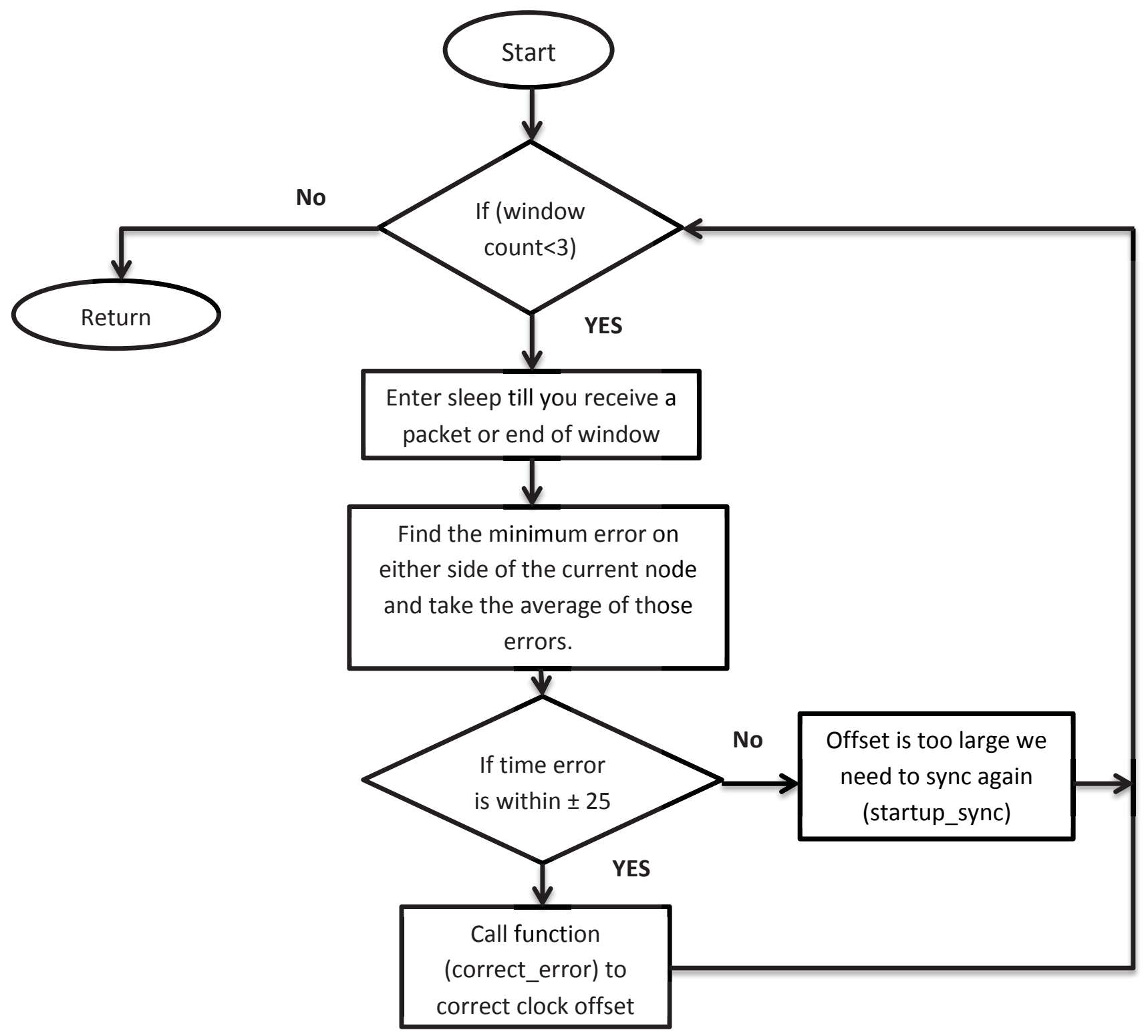


Flow chart 6: function 'normal_mode'

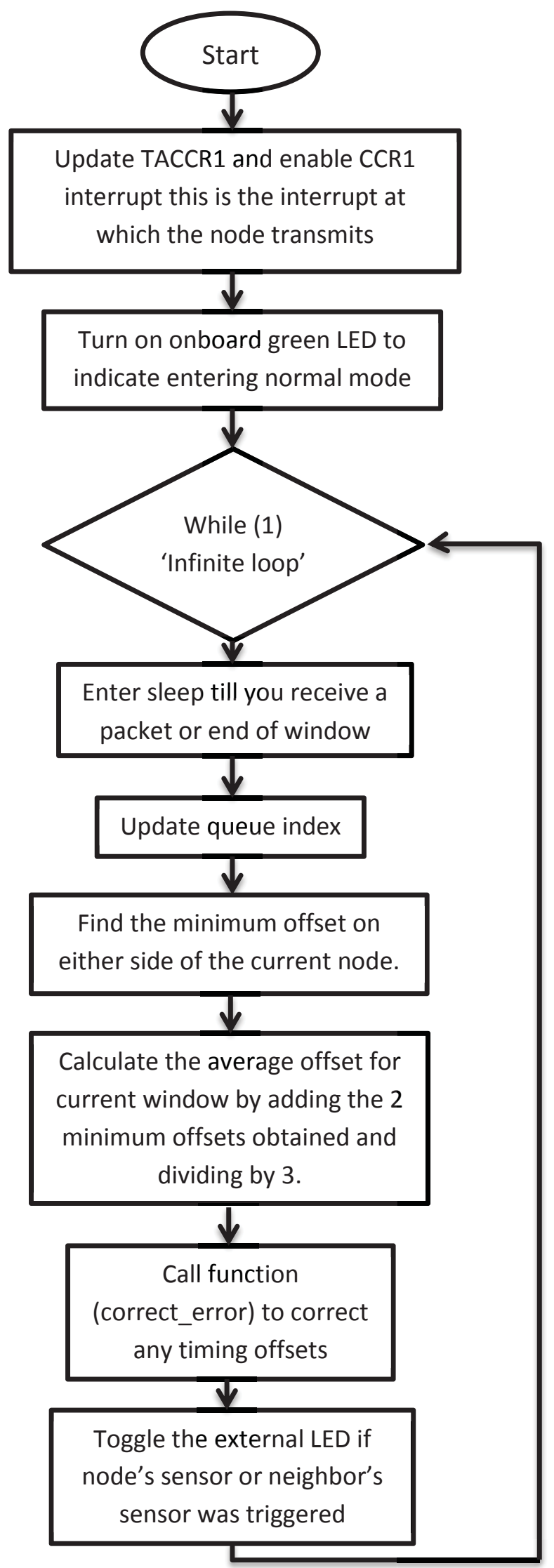


Appendix B

\section{User Manual}

Puneeth Ramesh

October, 31, 2014 


\section{Contents}

1 Introduction 45

2 Overview of the tool 45

$3 \quad$ Basic working $\quad 46$

3.1 Programming the Node's and the receiver node 46

3.2 Connecting to the receiver node 47

3.3 Changing the target node 48

3.4 Changing streams 49

3.5 Export collected error data to spread sheet 50

3.6 Monitoring sensor data 51

3.7 Disconnecting the receiver node 52

4 References $\quad 52$ 


\section{Introduction}

This document describes in detail the PC software used to monitor each node's activity. The tool helps monitor the sensor data and the clock offsets in each node. This provides real time information of each node's activity. The tool is designed to monitor data from all 16 nodes. The tool can be used to tap into any node and collect data. This is possible as the nodes relay data in both directions. This provides the flexibility to collect data from the first node, the last node or any node in-between. The tool was designed and programed using C\#. The tool is designed to be used in tandem with a eZ430-Rf2500 receiver node. The receiver node is programed to collect data from a target node and send the collected data to the tool via a UART to a USB port. The tool also sends instructions back to the receiver node. The instruction could be the desired target node or the upstream or downstream data from the desired target node. This document is a guide on how to use the tool to monitor and collect error data from the nodes.

\section{Overview of the tool}

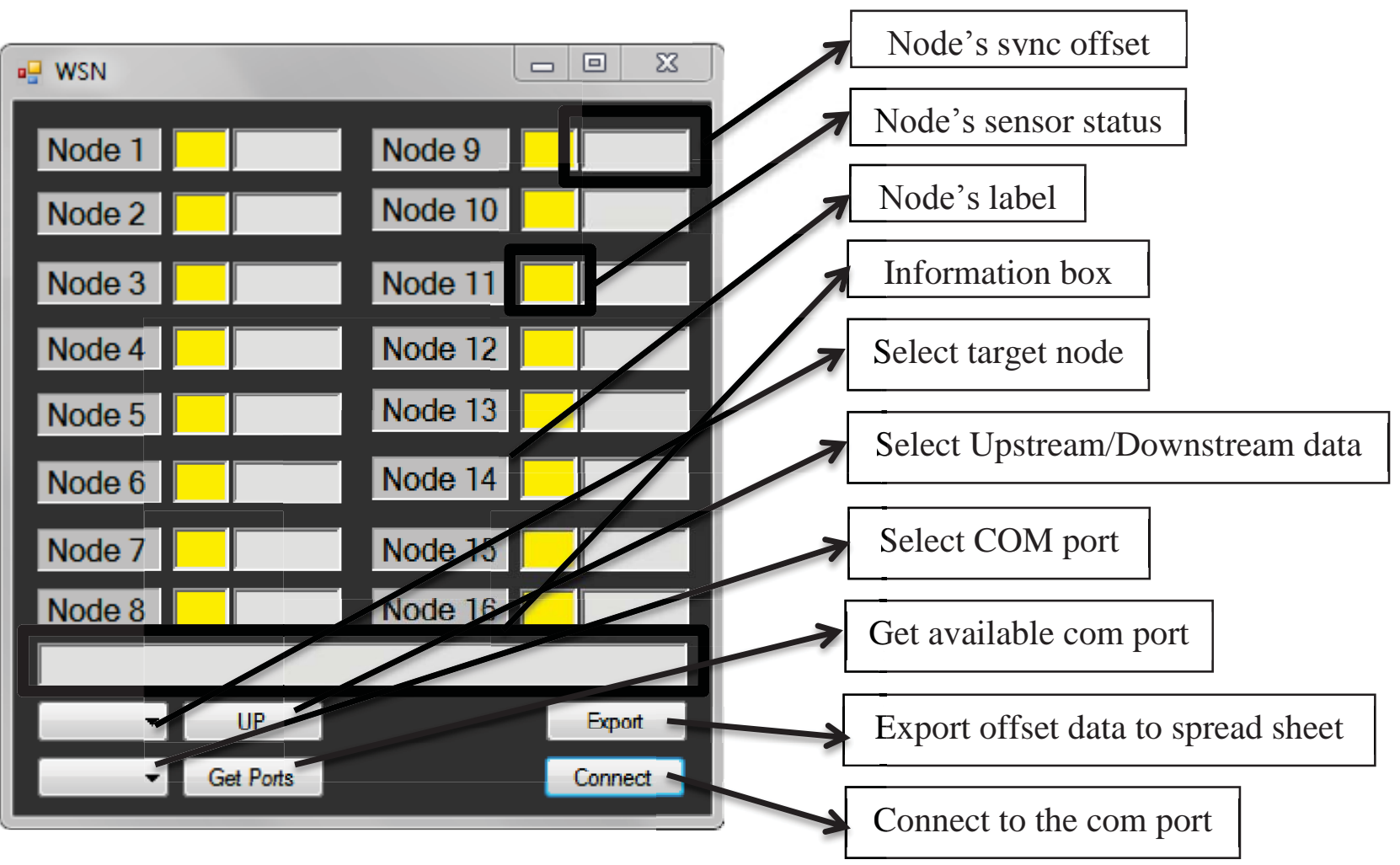

Figure 1. Basic layout of the tool's GUI. 


\section{Basic operation}

This section will describe how to use the tool. The basic layout of the graphical user interface (GUI) is shown in figure 1. Before we start using the GUI we need to program the receiver node with the receiver code using IAR embedded workbench; only then we can communicate with the receiver node. Once the receiver node is up and running we can now start the tool by double clicking on the "WSN.exe" file. First the tool needs to connect to the receiver node. Since serial communication (UART) is being used, we need to connect to the appropriate COM port on the PC.

\subsection{Programming the network nodes and the receiver node.}

This manual assumes that the user has IAR and the required drivers installed on his computer. To program the nodes the user needs 2 files ("node.c" and "wireless.h"). Place both the files in the same project folder and select the device under project options as shown in figure 2. Before programming the device, update the node address in the main function as shown in figure 3. The node address has to be changed manually for each and every node. Then click on the "Download and Debug" button to program the device as shown in figure 4.

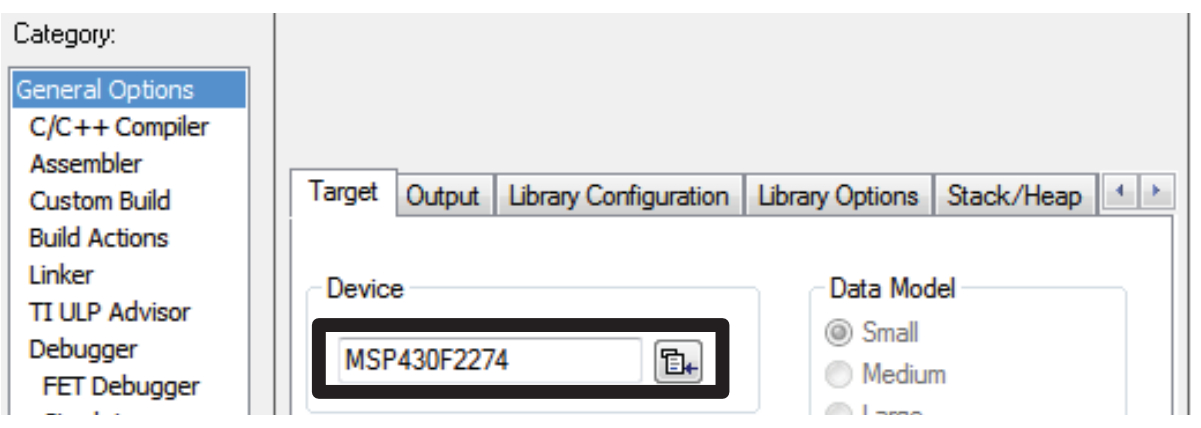

Figure 2. Select the device under project options.

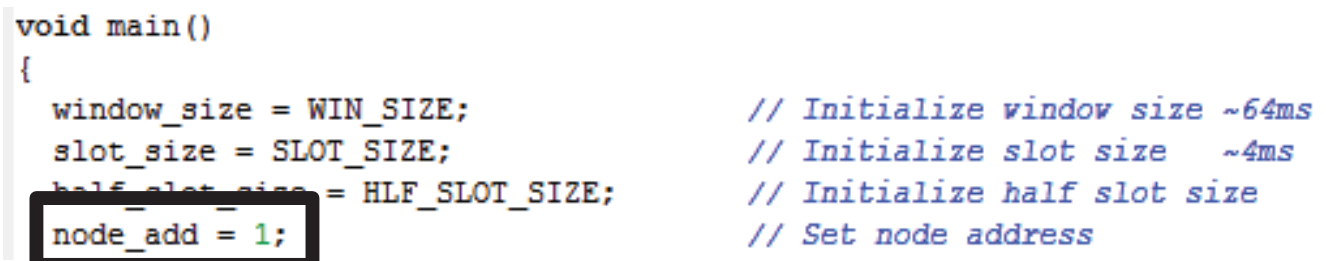

Figure 3. Update the Node address in the main function of the code. 


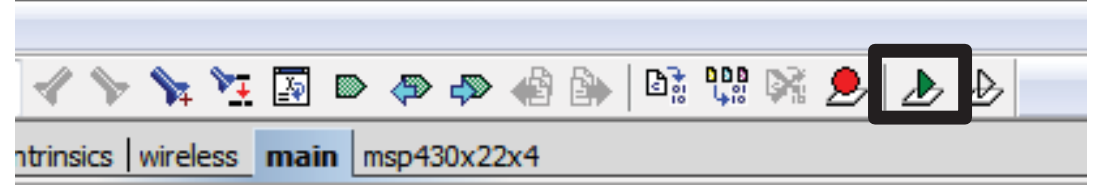

Figure 4. Click on the download and debug button to program the device.

The receiver node is programmed in the same way. The receiver node also requires two files ("receiver_node.c" and "wireless.h") to program it. The wireless.h file is the same for both.

\subsection{Connecting to the receiver node}

First, plugin the receiver node to a USB port and then to find the receiver node's COM port. On your desktop click start. In the search bar type Device Manager. Then open Device Manager. This will give you the window shown in figure 5. Under ports we can find the receivers' COM port. In figure 5 the COM port is COM21.

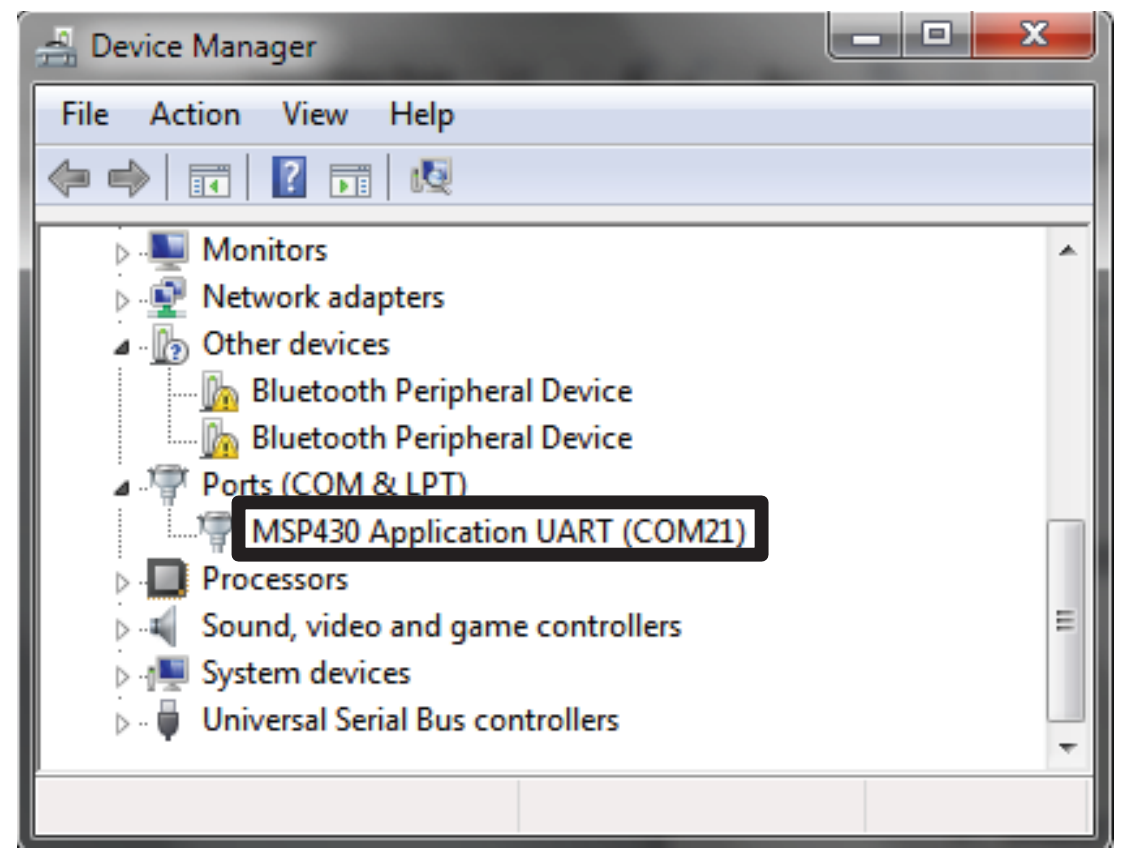

Figure 5. Device Manager showing active COM ports.

Once the COM port is known we can now connect to the receiver node. On the tool's GUI click the "Get Ports" button to generate a list of active COM ports on your PC and select the receivers' COM port as shown in figure 6. 


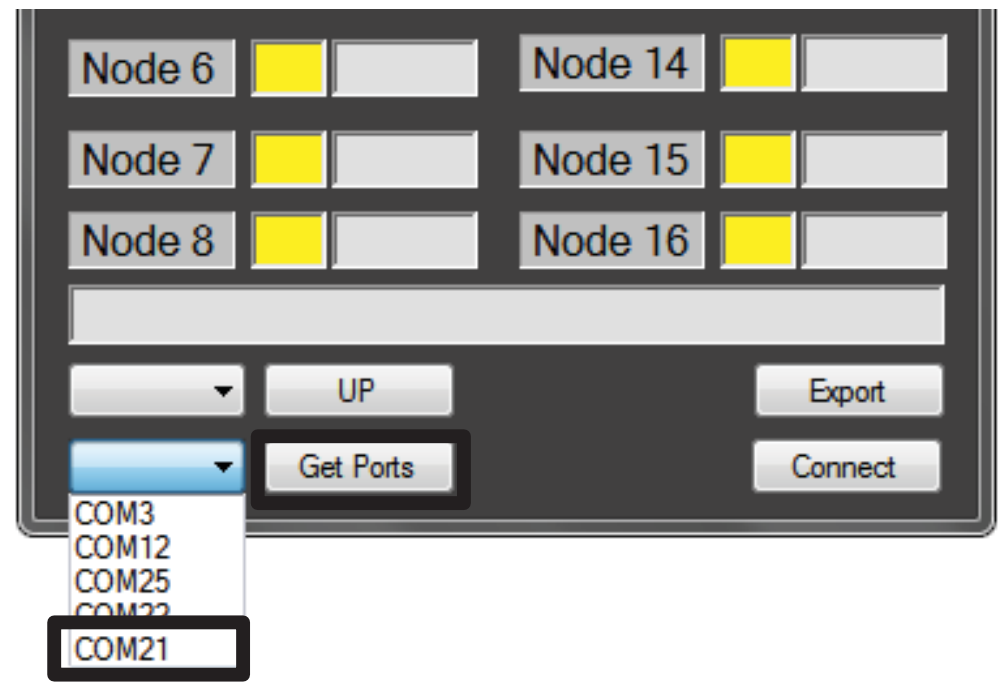

Figure 6. Selecting COM port on the GUI.

Once we select the COM port we can now hit the "Connect" button to establish a connection. Once the connection is established we get a confirmation in the information box as shown in figure 7. We cannot establish a connection without selecting the COM port. This will cause an error message on the information box.

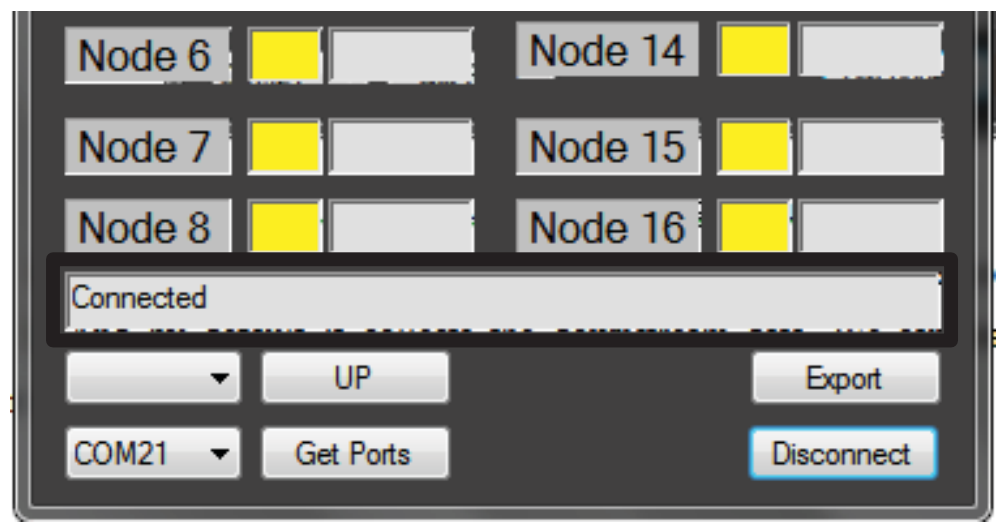

Figure 7. After establishing connection.

\subsection{Changing the target node}

Once the connection is established by default the tool starts collecting data from node 1 . And by default it collects the upstream data and starts monitoring the sensor data. We can change the target node by selecting a different node from the drop down list next to the "UP" button as shown in figure 8. This can only be done once a 
connection is established. Changing the target node before establishing a connection will result in a warning message in the info box.

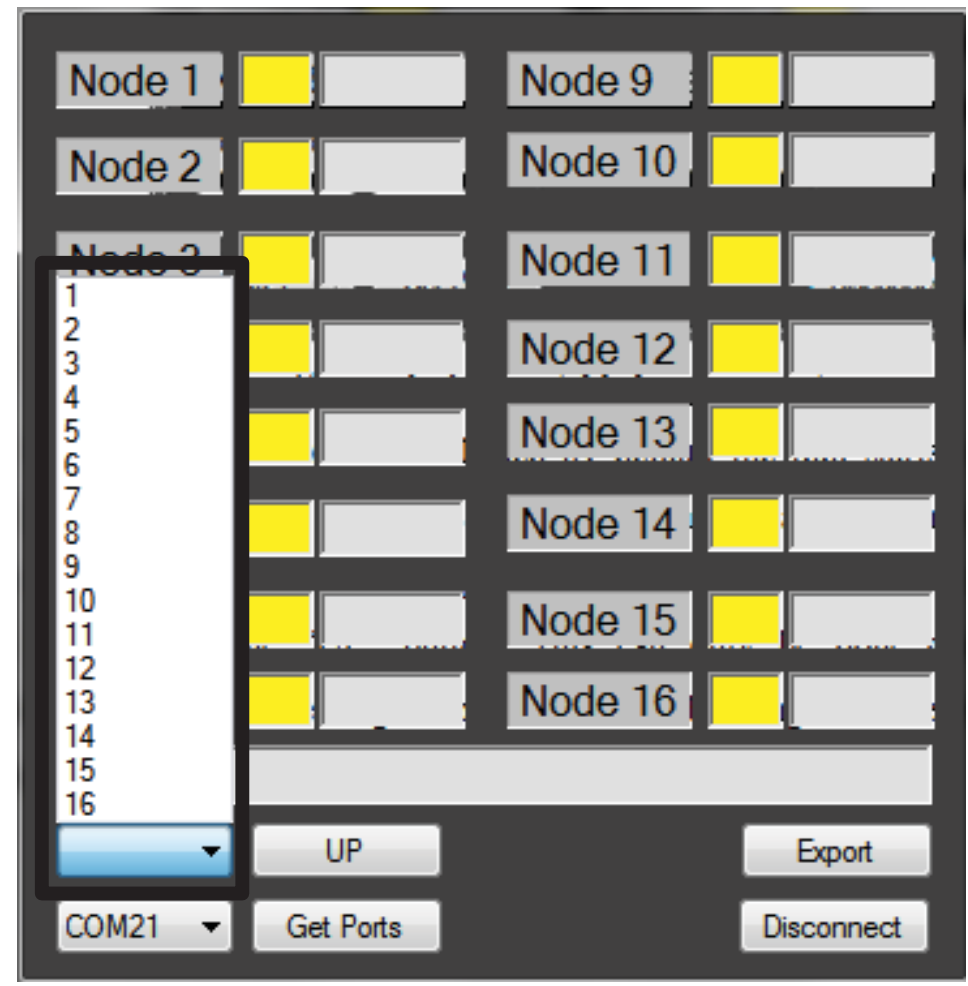

Figure 8. Changing the target node.

\subsection{Changing streams}

We can change streams by clicking the "UP/DOWN" button, as shown in figure 9. It is useful to change streams when our target node is the $16^{\text {th }}$ node, as all the data flows downstream.
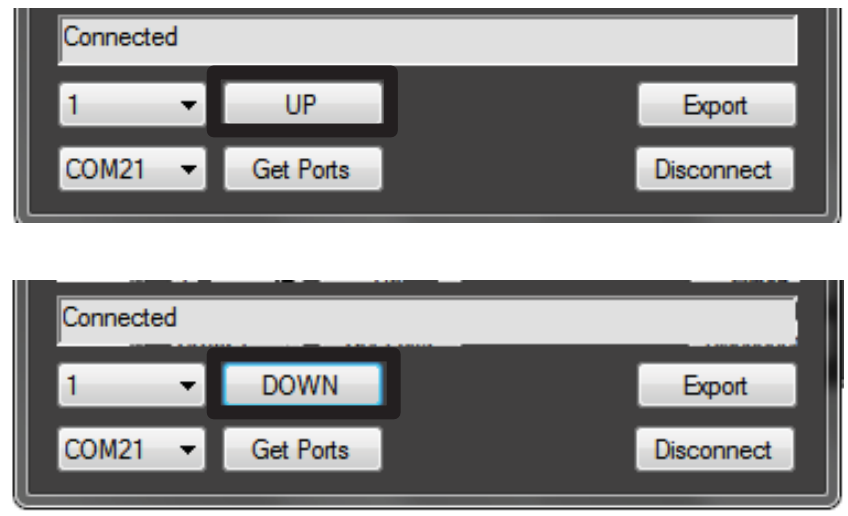

Figure 9. Collecting Upstream/Downstream data. 


\subsection{Export collected error data to spread sheet}

The tool has the capacity to store the previous 300 sync offsets for all 16 nodes. This can be dumped into a spread sheet. Later on the data can be plotted. To save the data to a spread sheet we just need to hit the "Export" button as shown in figure 10. This will create or overwrite a csv file with the name "Output.csv". It is recommended to disconnect before exporting the data.

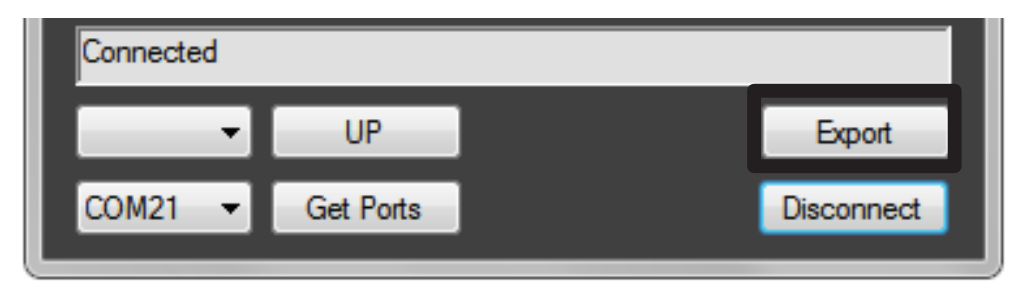

Figure 10. Export to spread sheet.

The data saved to the spread sheet is as shown in figure 11. This can be plotted to see the variation with respect to nominal.

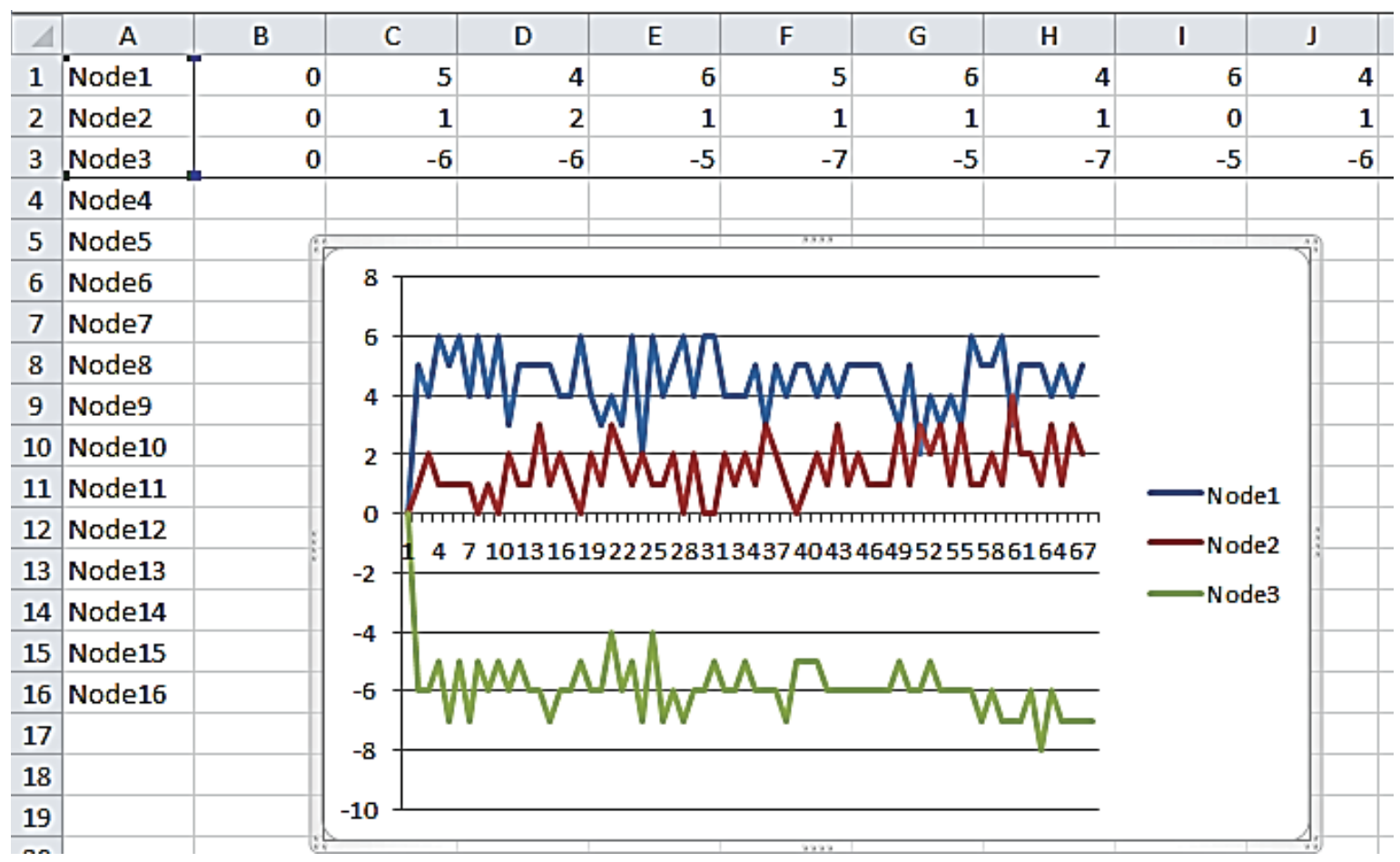

Figure 11. Exported data. 


\subsection{Monitoring sensor data}

The sensor data from each node can be monitored from the small color window next to the node labels as shown in figure 12. The meaning of each color is shown in figure 13.

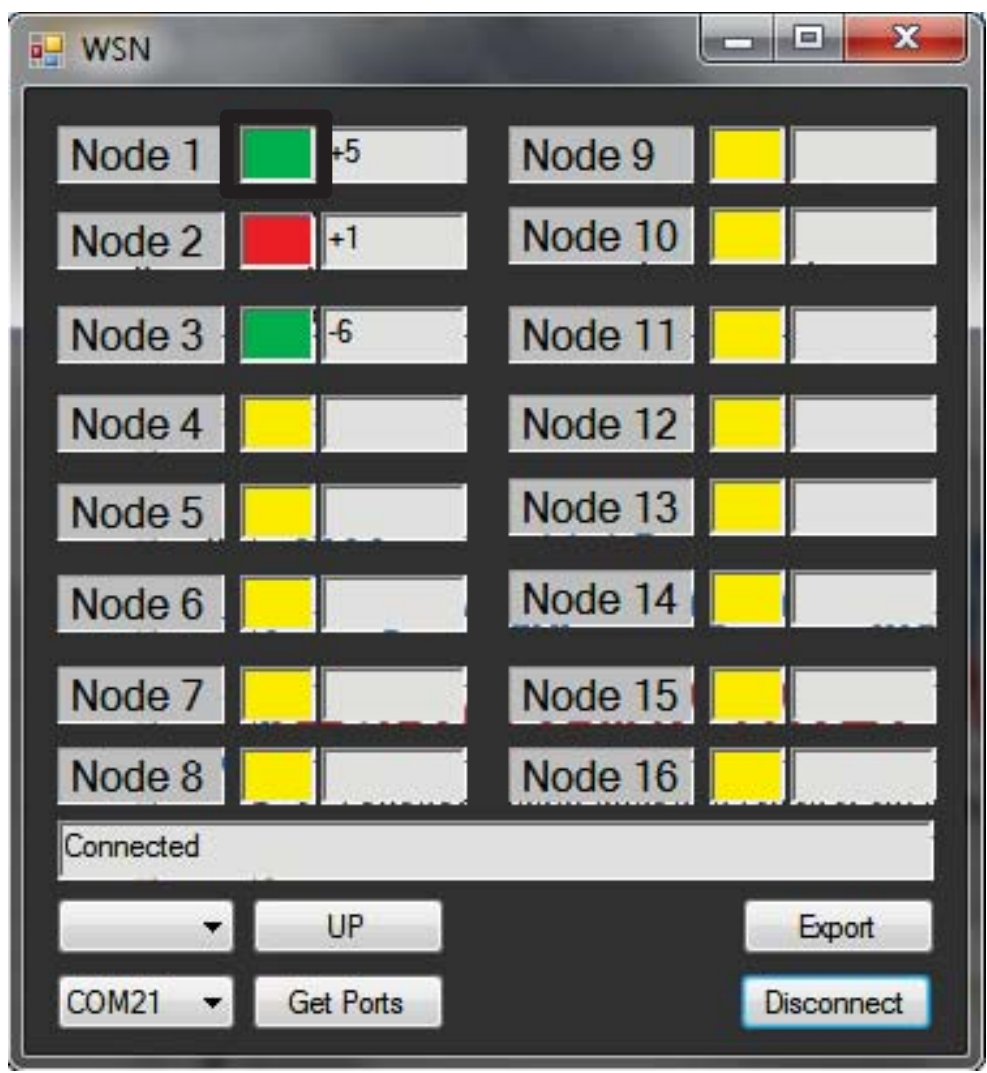

Figure 12. Monitoring sensor data.

The sensor is offline.

The sensor is online and no motion was detected.

The sensor is online and motion was detected.

Figure 13. Sensor status. 


\subsection{Disconnecting the receiver node}

To disconnect the receiver node from the tool hit the "Disconnect" button (as shown in figure 14) or closing the window with the "X" button at the top will also safely disconnect the receiver node (close's the COM port). Caution: Force closing the tool from task manager or any other tool will lock the COM port.

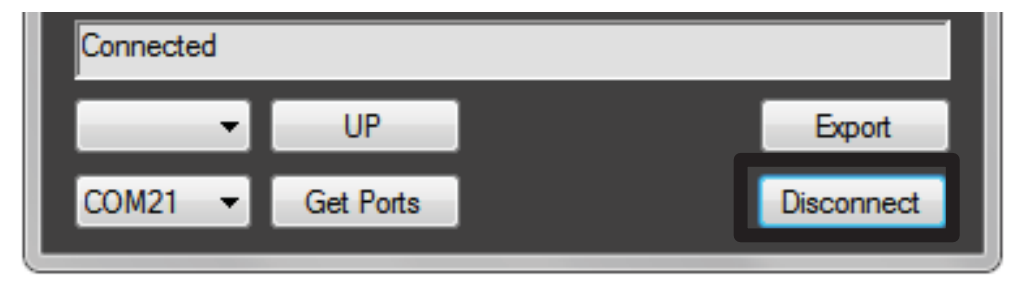

Figure 14. Disconnecting the receiver node.

\section{References}

[1] Microsoft Visual Studio 2013, C\# Programming Guide. Accessed on: June 2014, Available: http://msdn.microsoft.com/en-us/library/67ef8sbd.aspx.

[2] Microsoft Developer Network, SerialPort Class. Accessed on: June 2014, Available: http://msdn.microsoft.com/en-us/library/system.io.ports.serialport\% 28v =vs. $110 \% 29$.aspx.

[3] Instructables Tutorial, Serial Port Programming With .NET. Accessed on: June 2014, Available: http://www.instructables.com/id/Serial-Port-Programming-WithNET/step1/Set-up-and-Open-the-Serial-Port/

[4] Texas instruments, MSP430x22×2, MSP430x22x4 Mixed Signal microcontroller Datasheet, 2012. , Available: http://www.ti.com.cn/cn/lit/ds/symlink/msp430f2274.pdf.

[5] Texas instruments, CC2500 Low-Cost Low-Power 2.4 GHz RF Transceiver Datasheet, 2014. , Available: http://www.ti.com/lit/ds/symlink/cc2500.pdf.

[6] Texas instruments, eZ430-RF2500 Development Tool User guide. SLAU227A Mixed Signal Products, 2007. , Available: http://www.ti.com/lit/ug/slau227e/slau227e.pdf. 


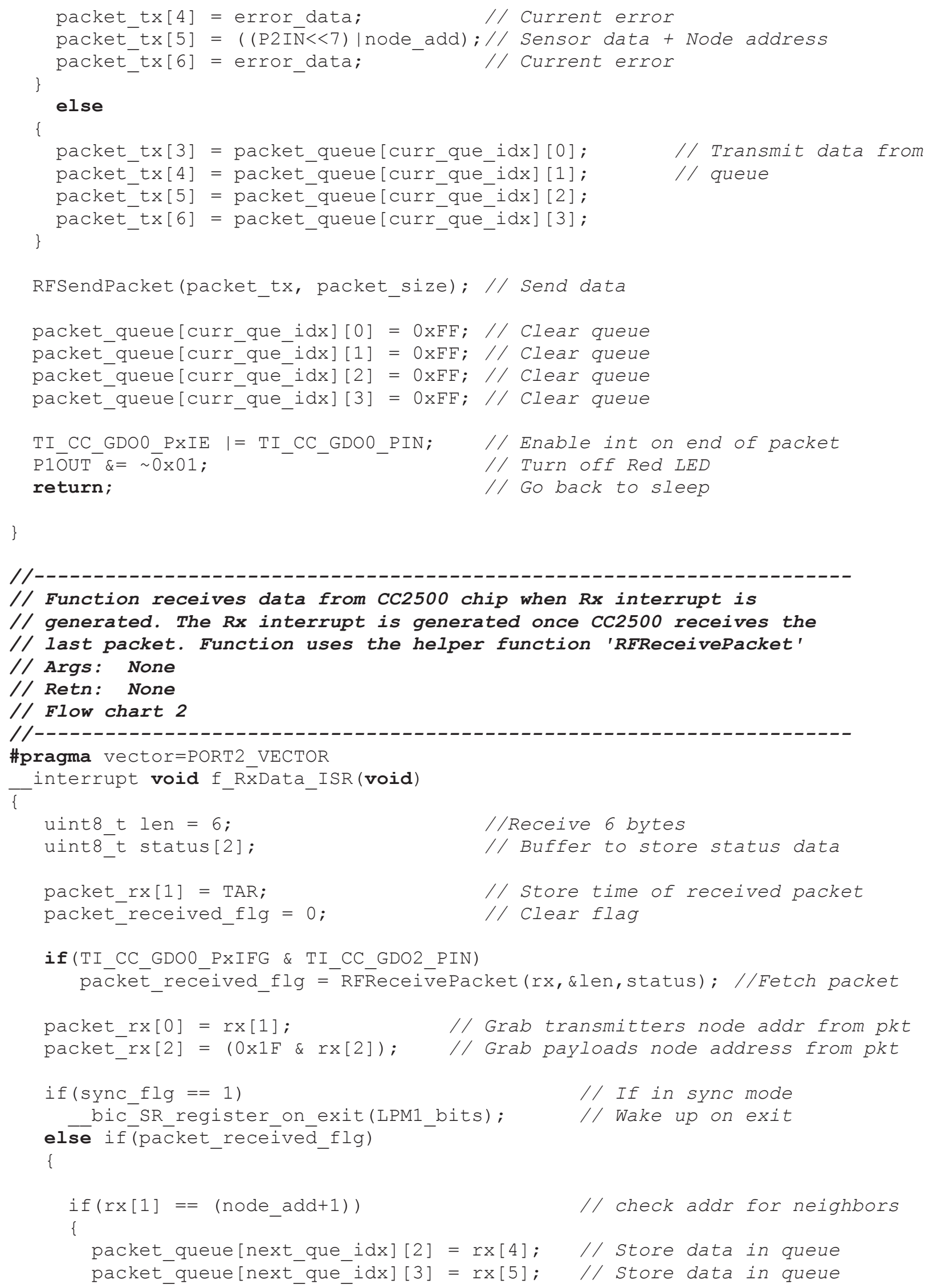




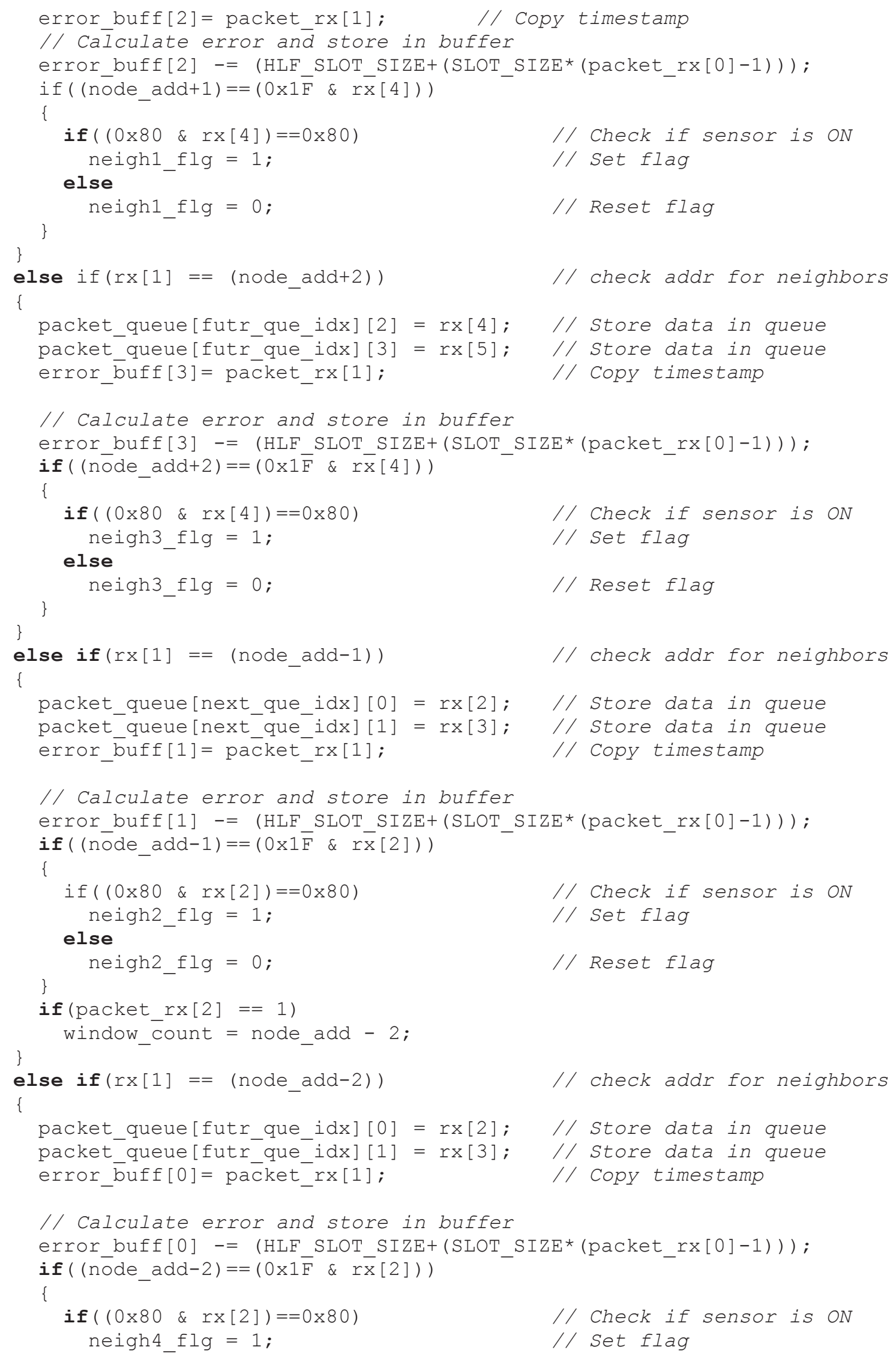


Node Source Code $\mid 56$

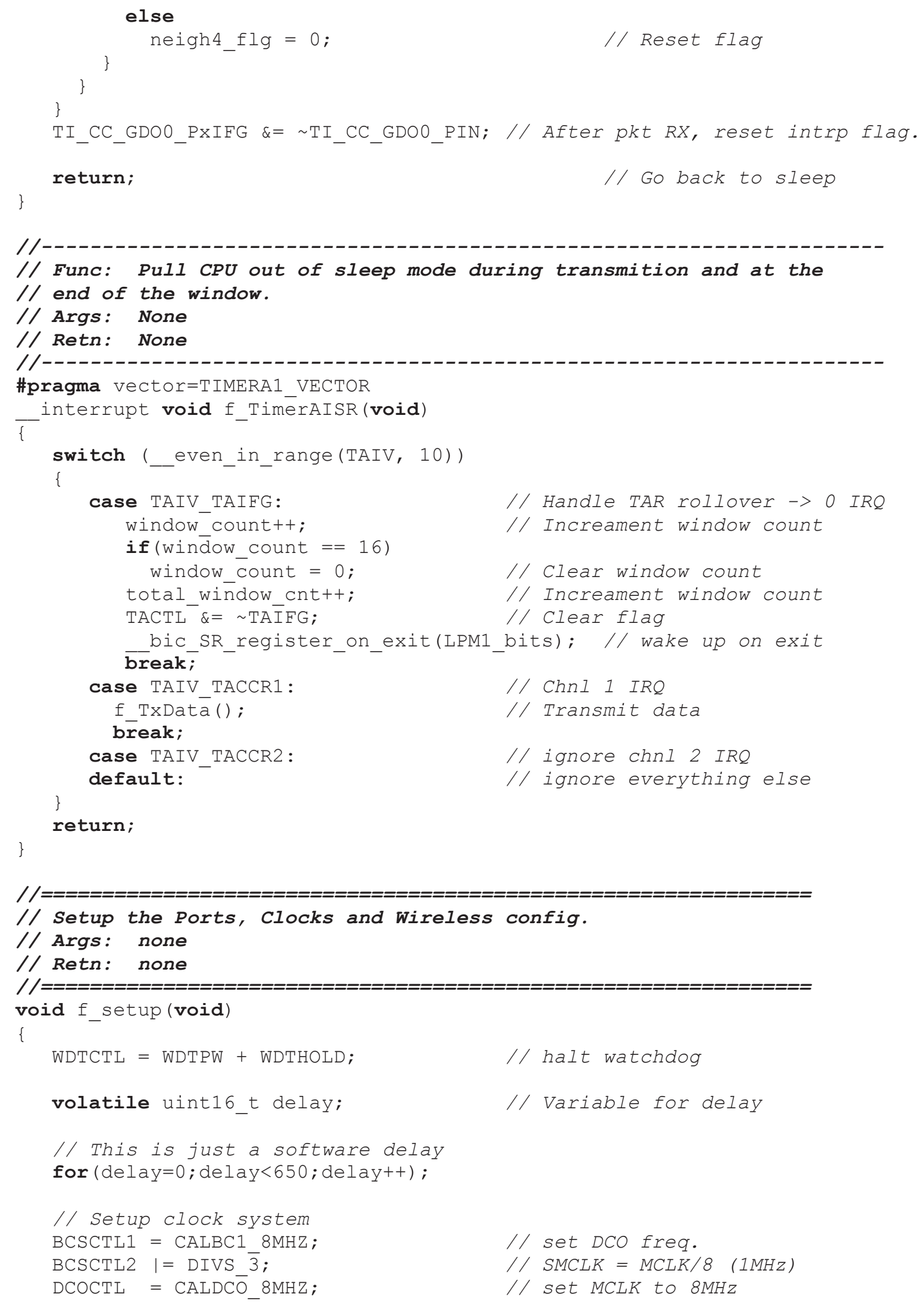




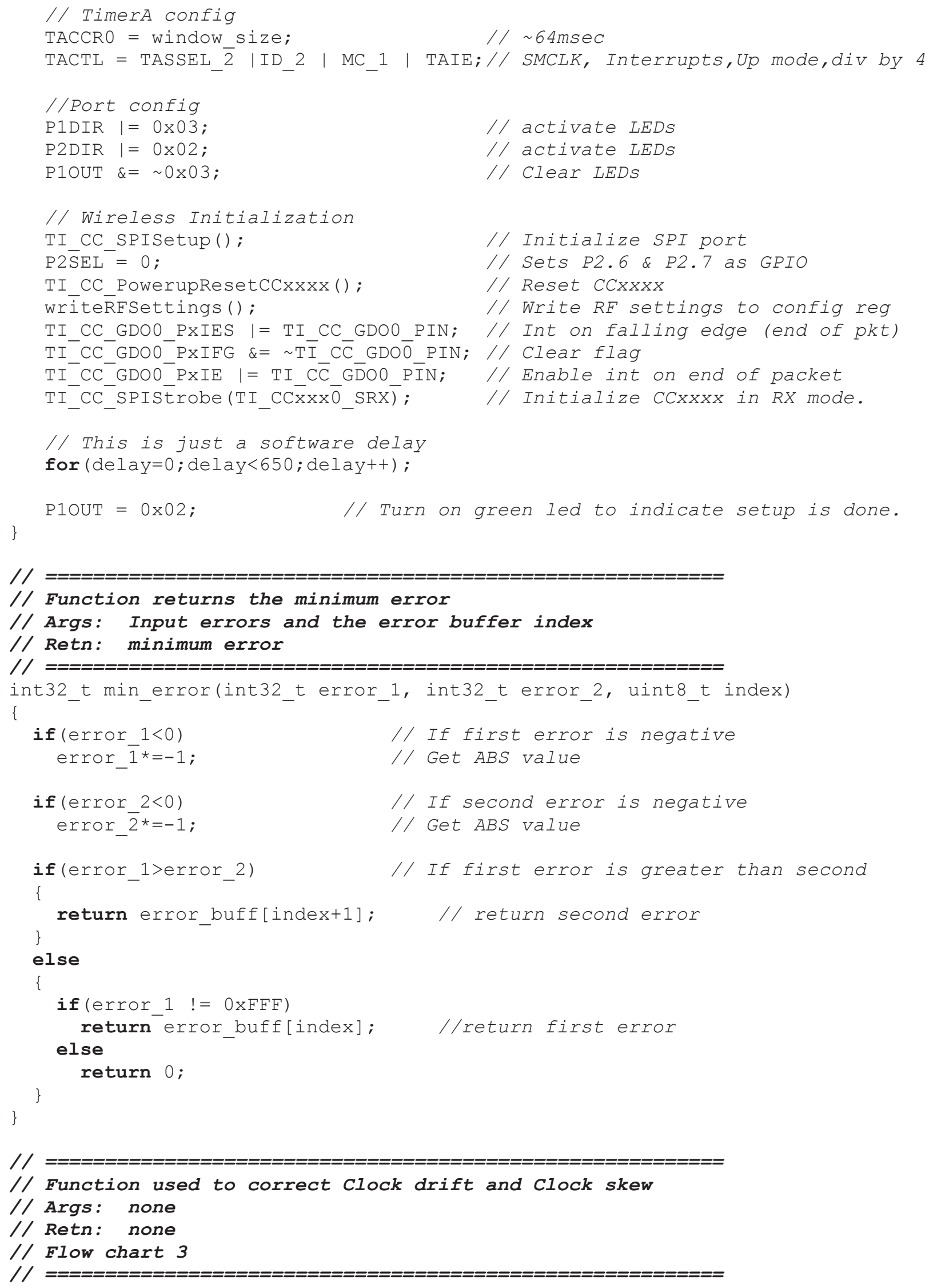




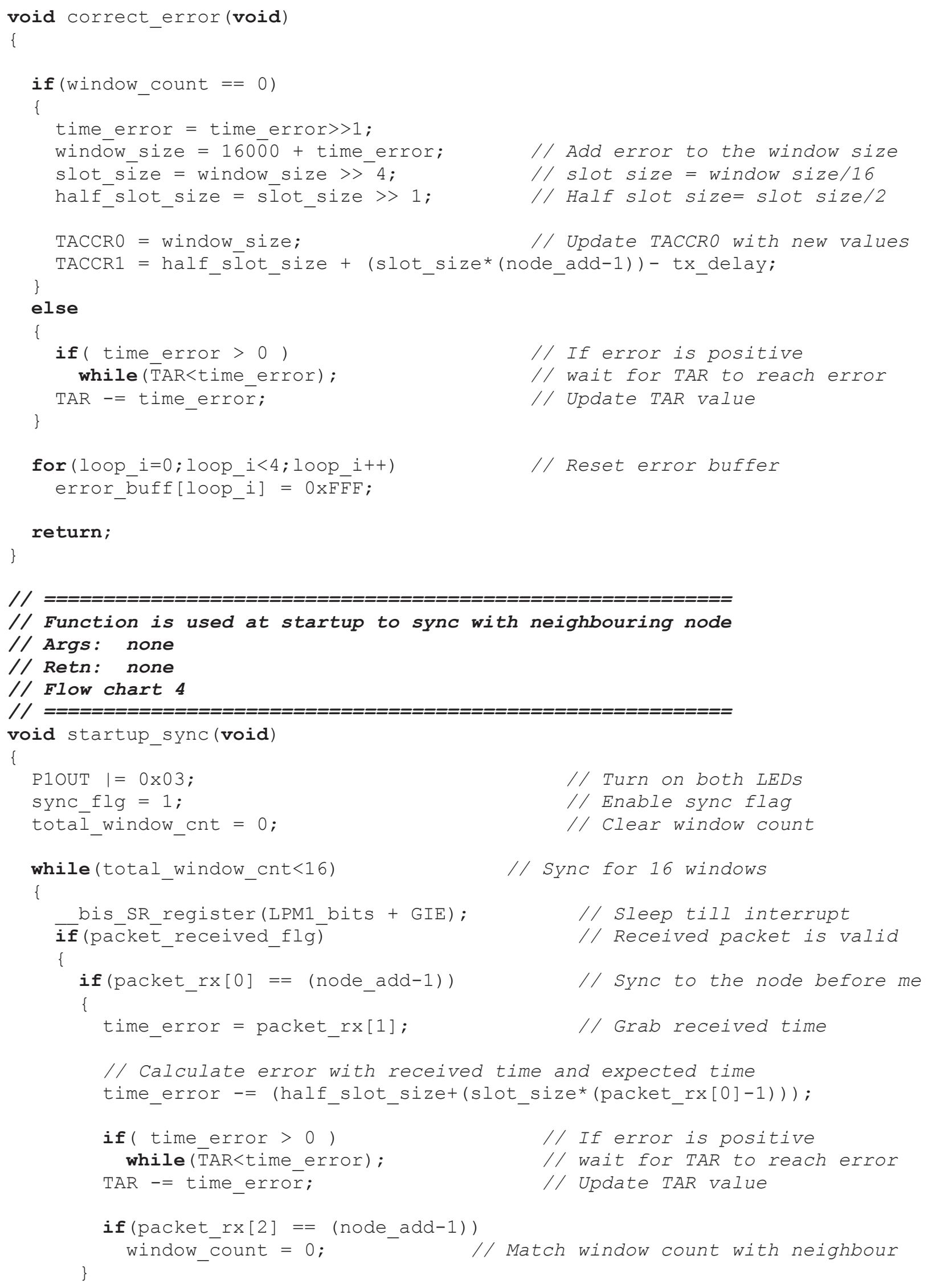


Node Source Code | 59

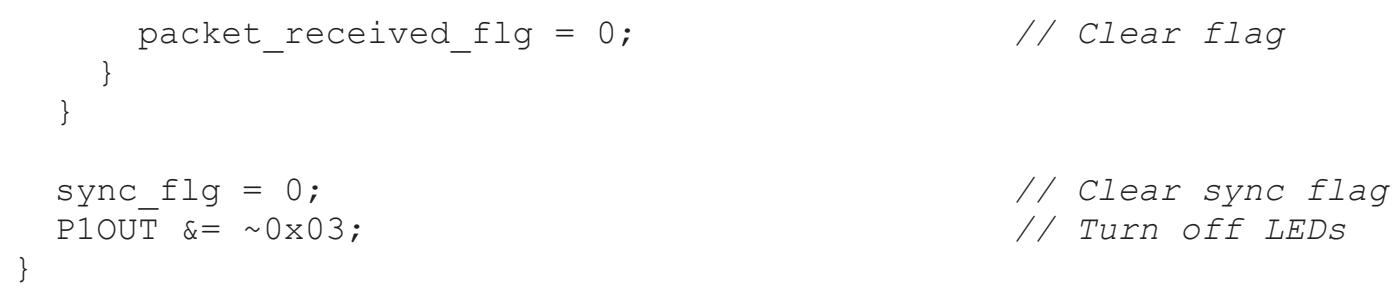




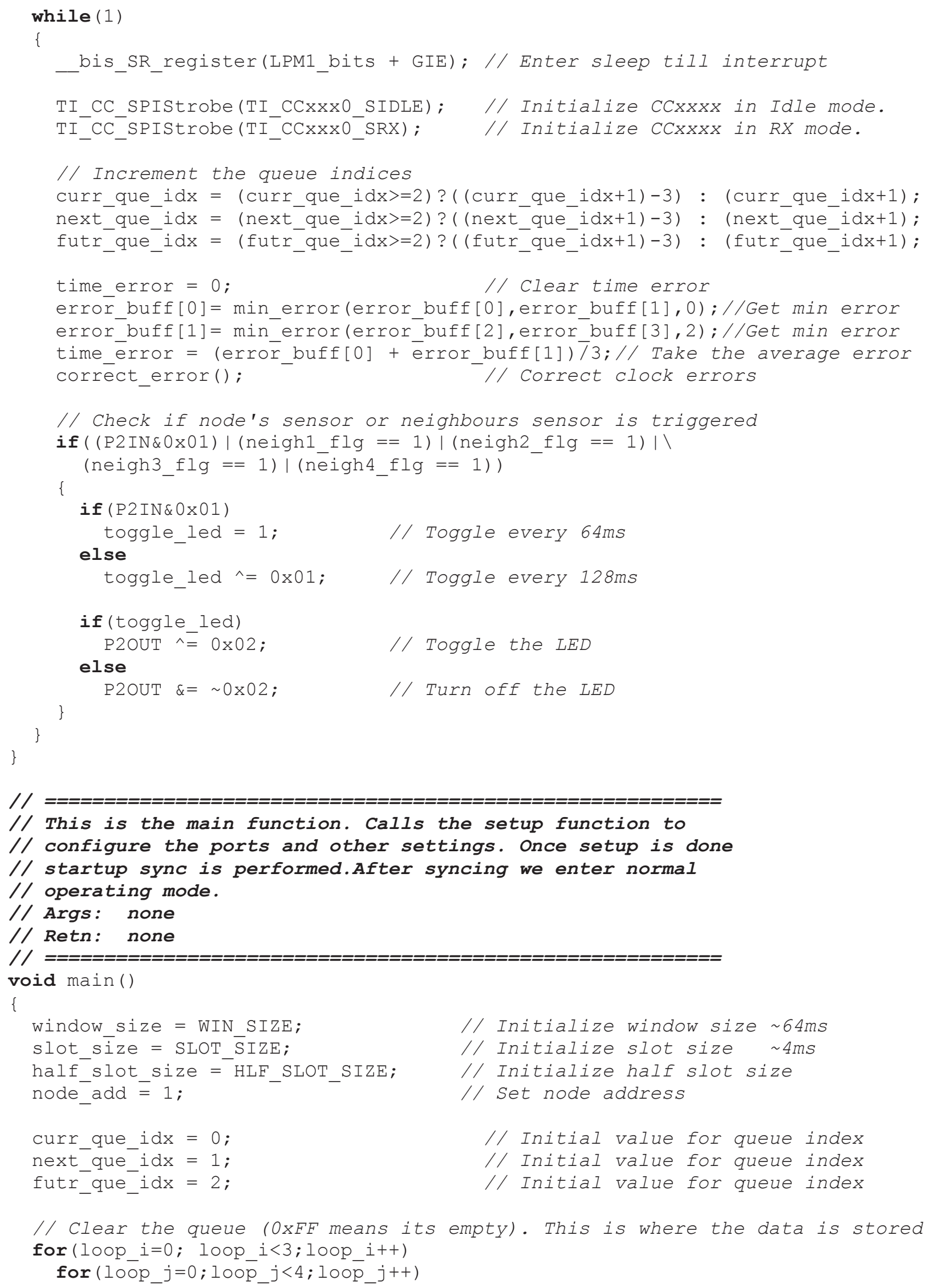


Node Source Code | 61

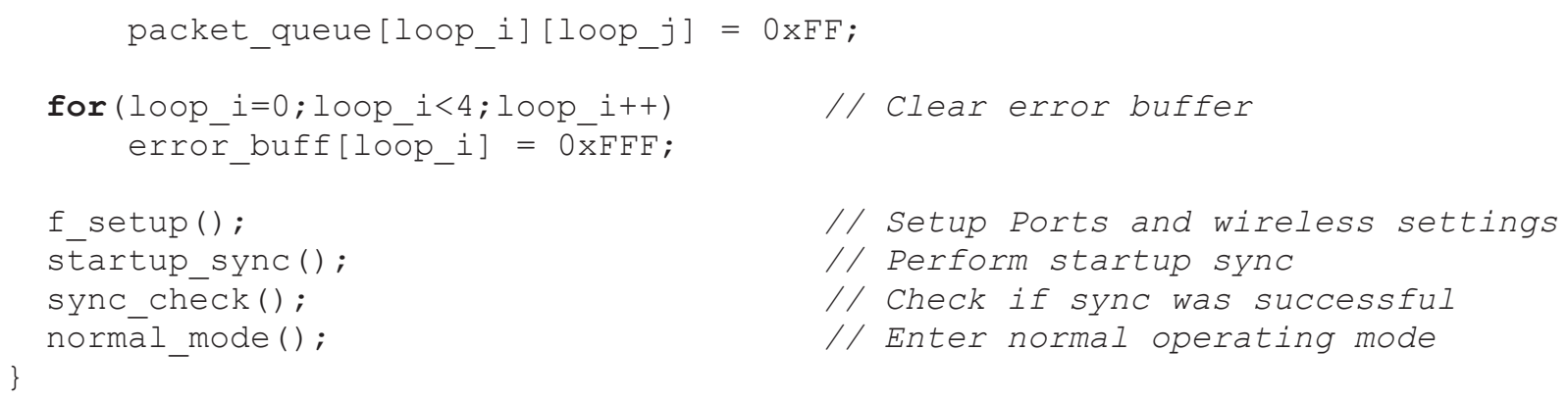




\section{Appendix D}

\section{Receiver Node Source Code:}

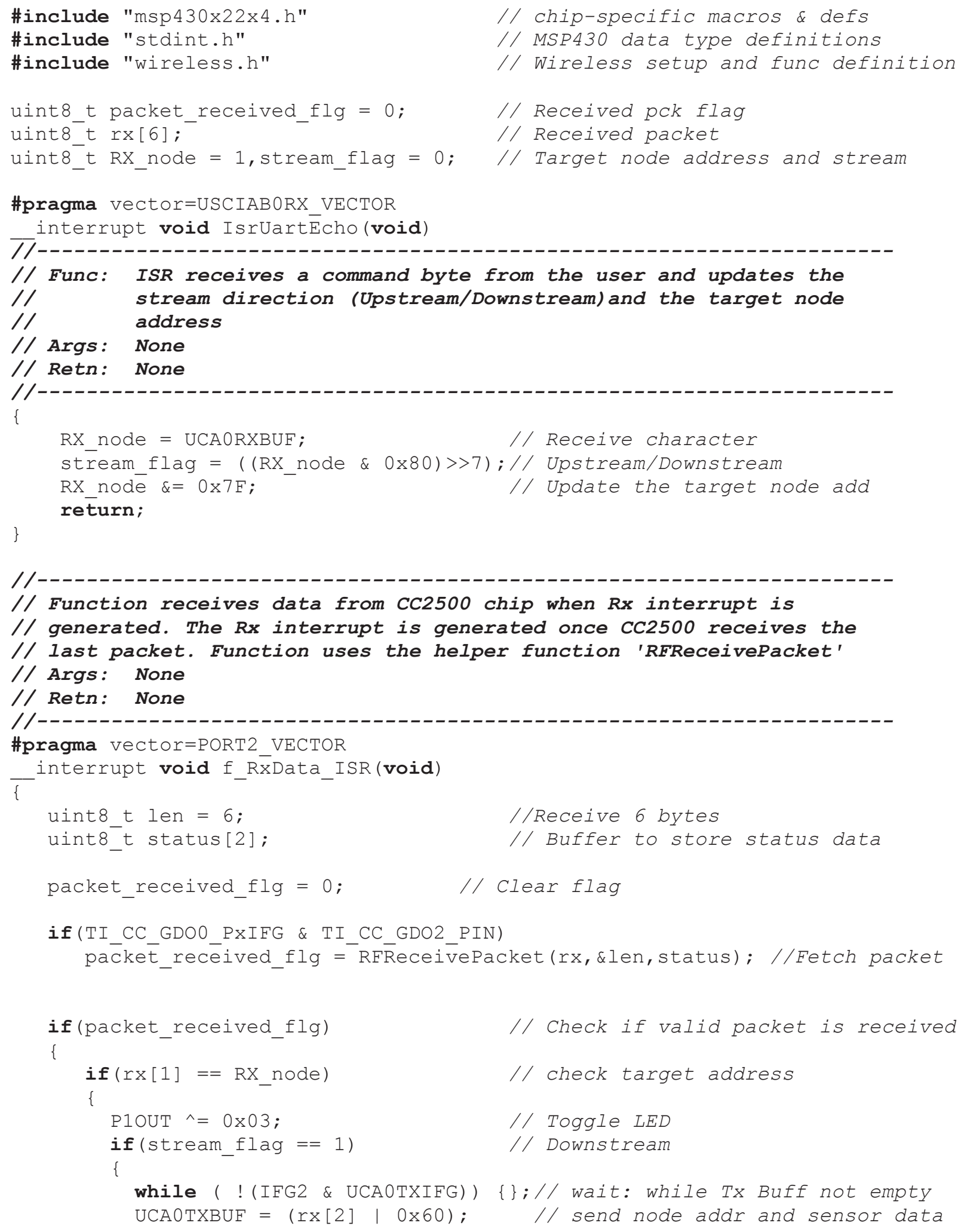




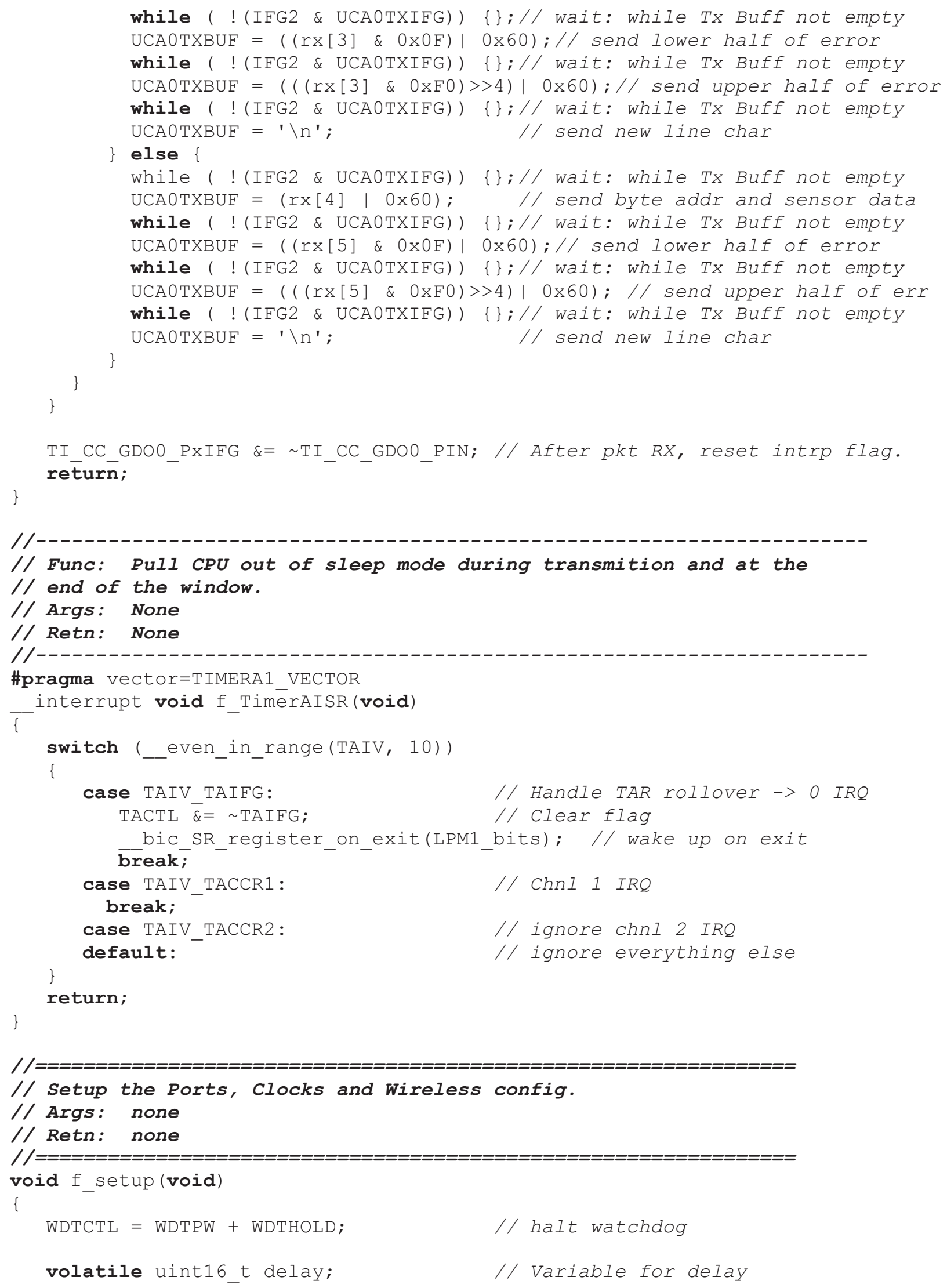




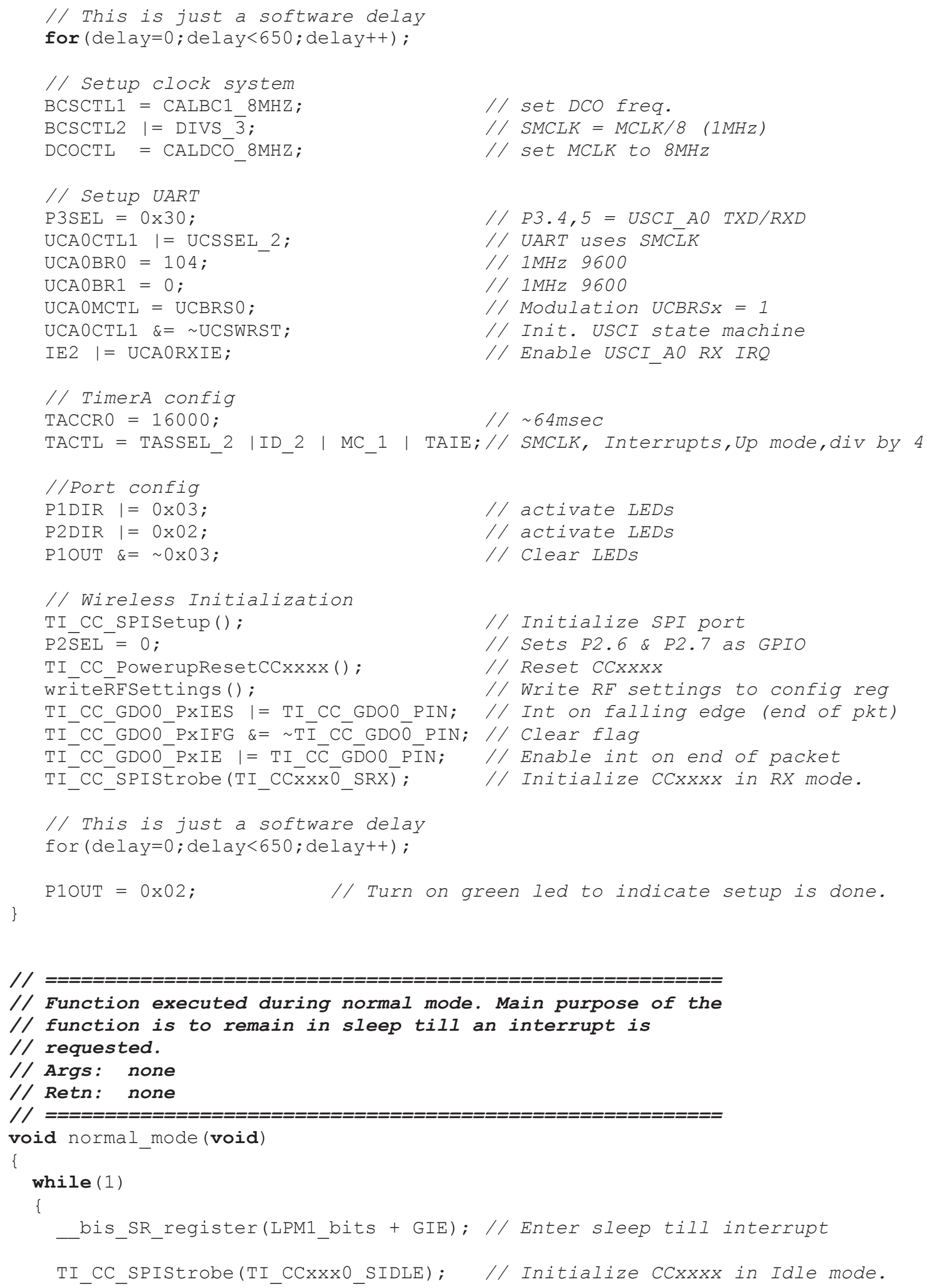


Receiver Node Source Code $\mid 65$

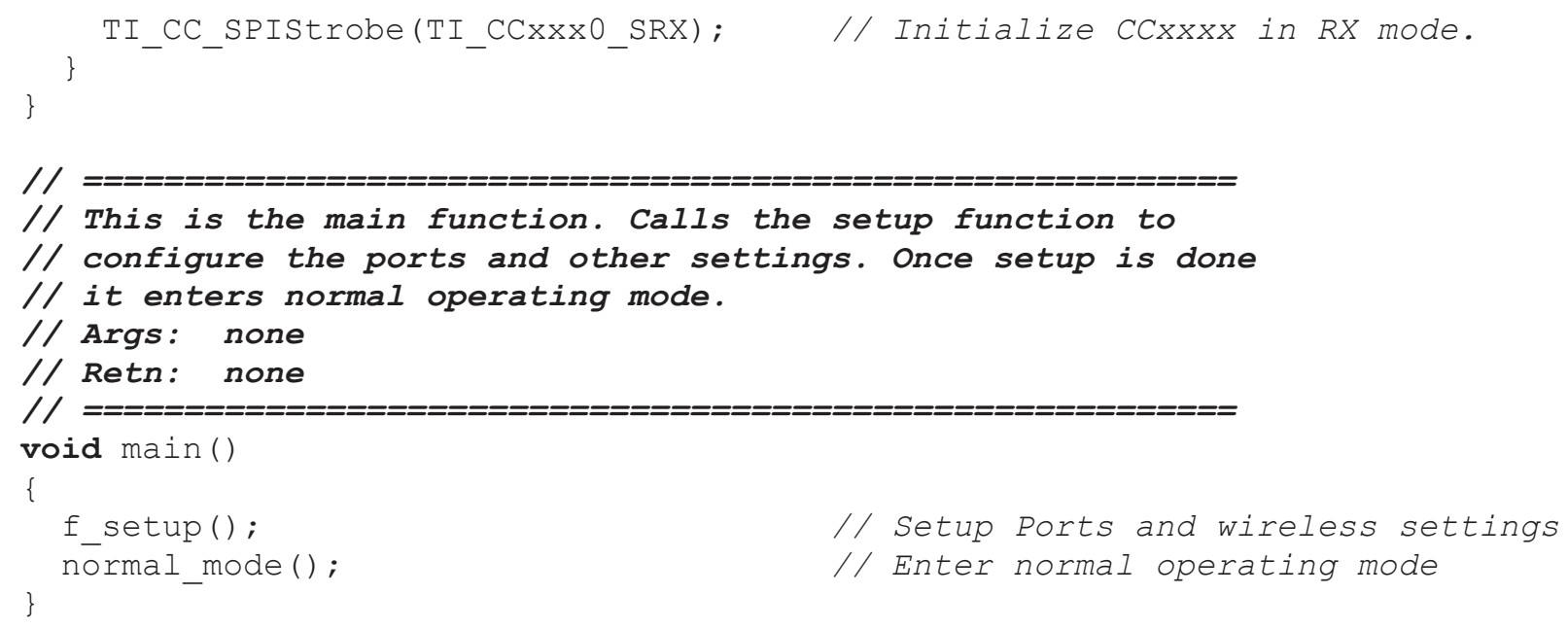




\section{Appendix E}

\section{GUI Source Code:}

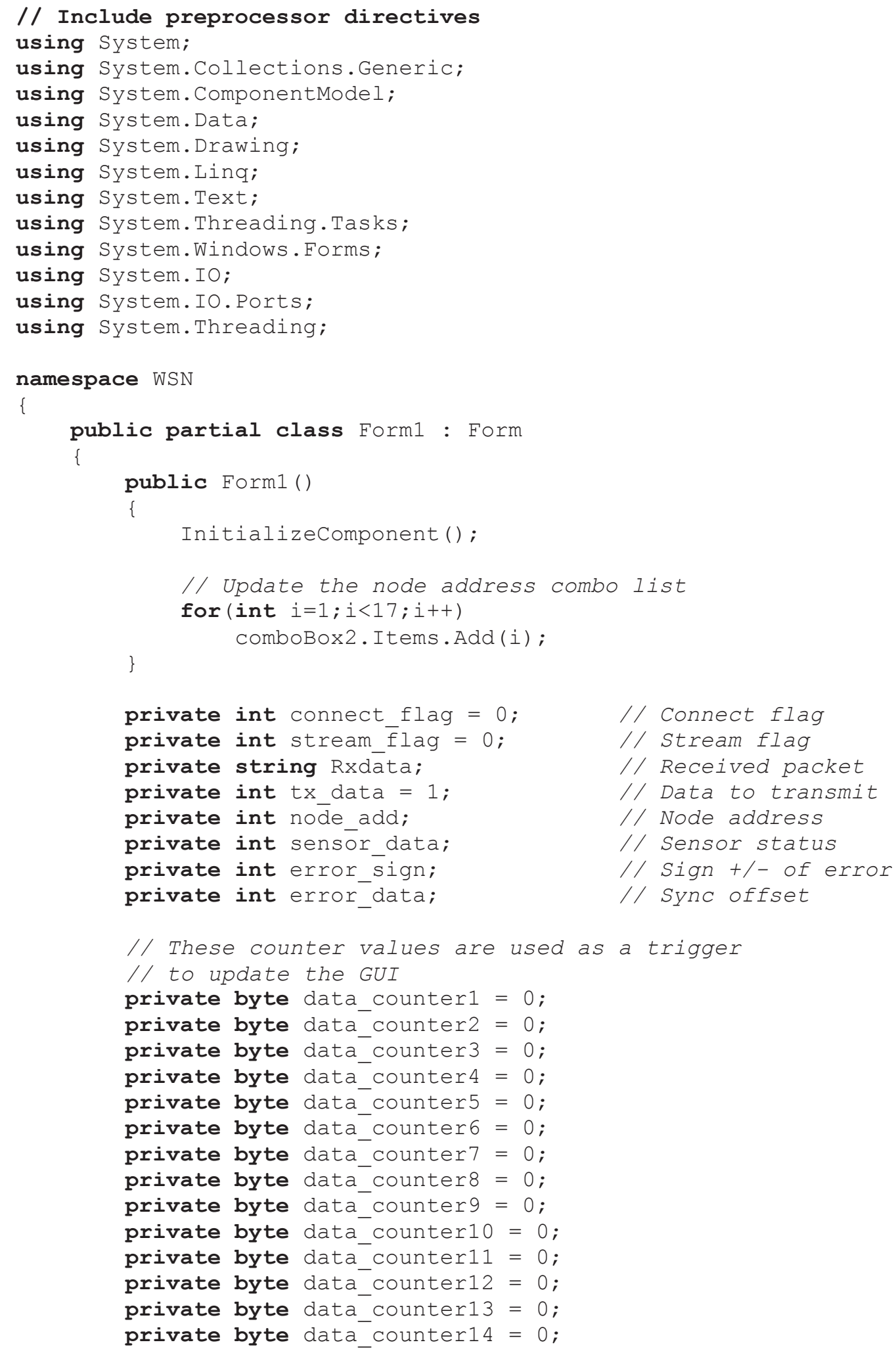




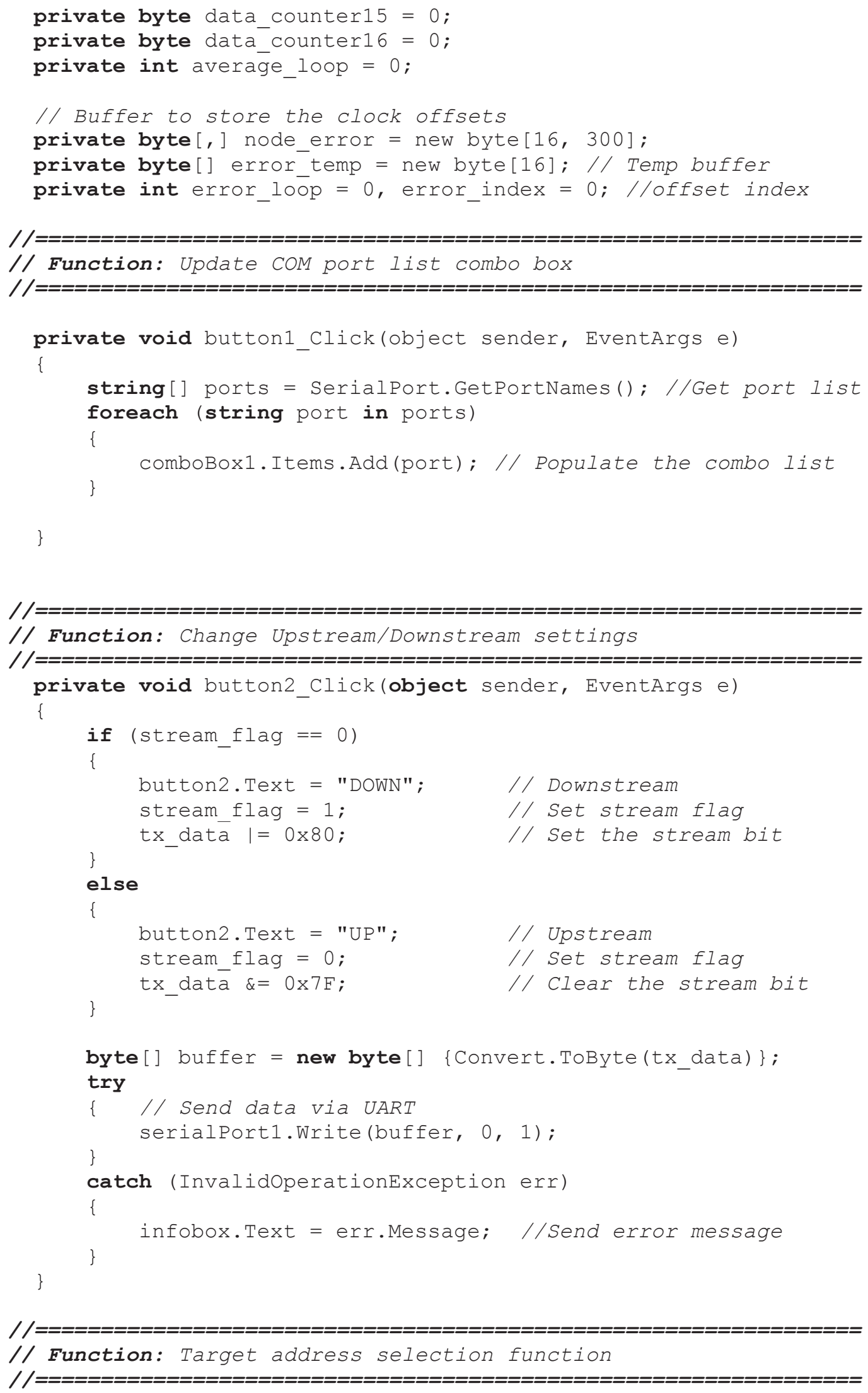




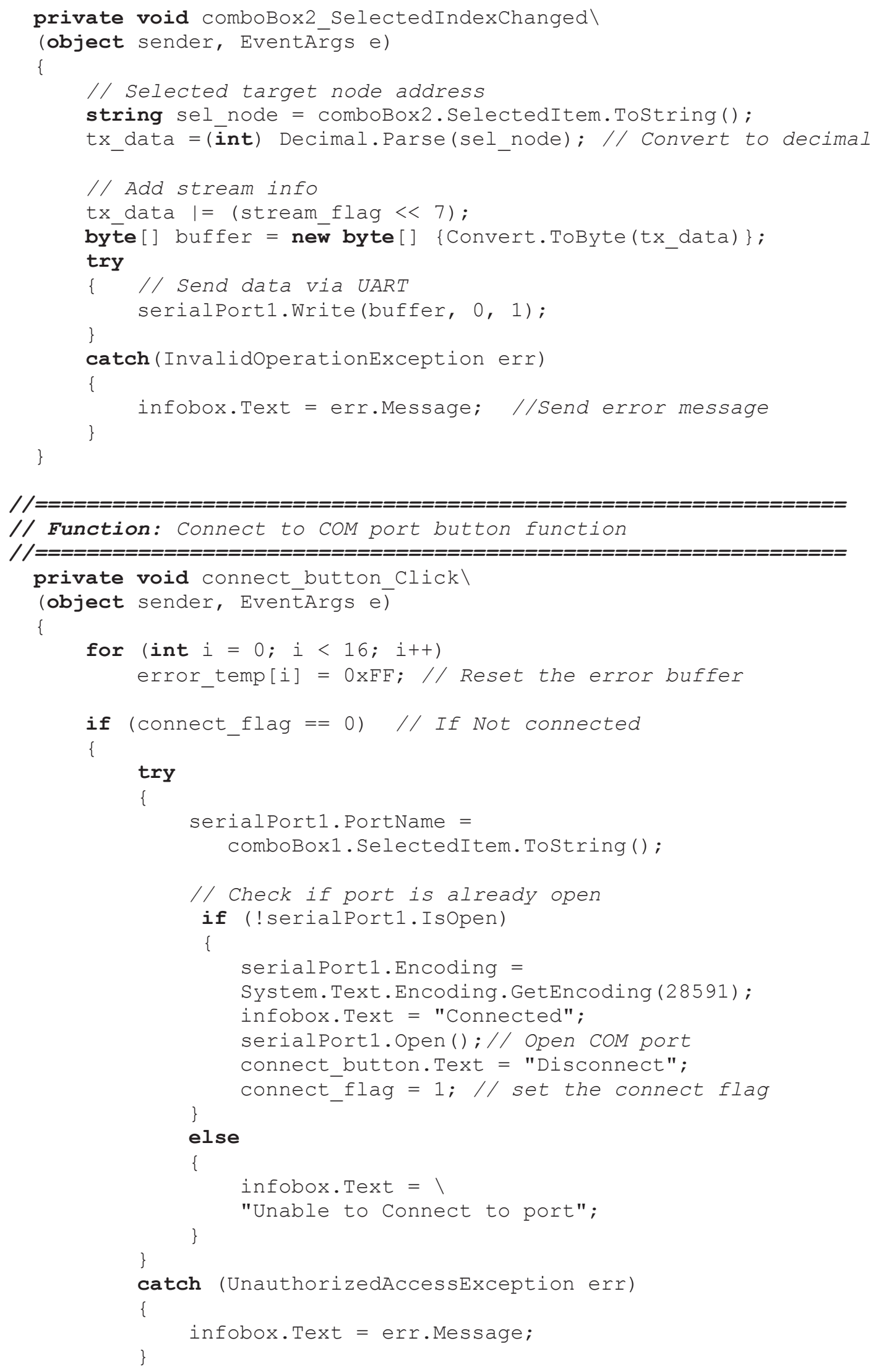


GUI Source Code | 69

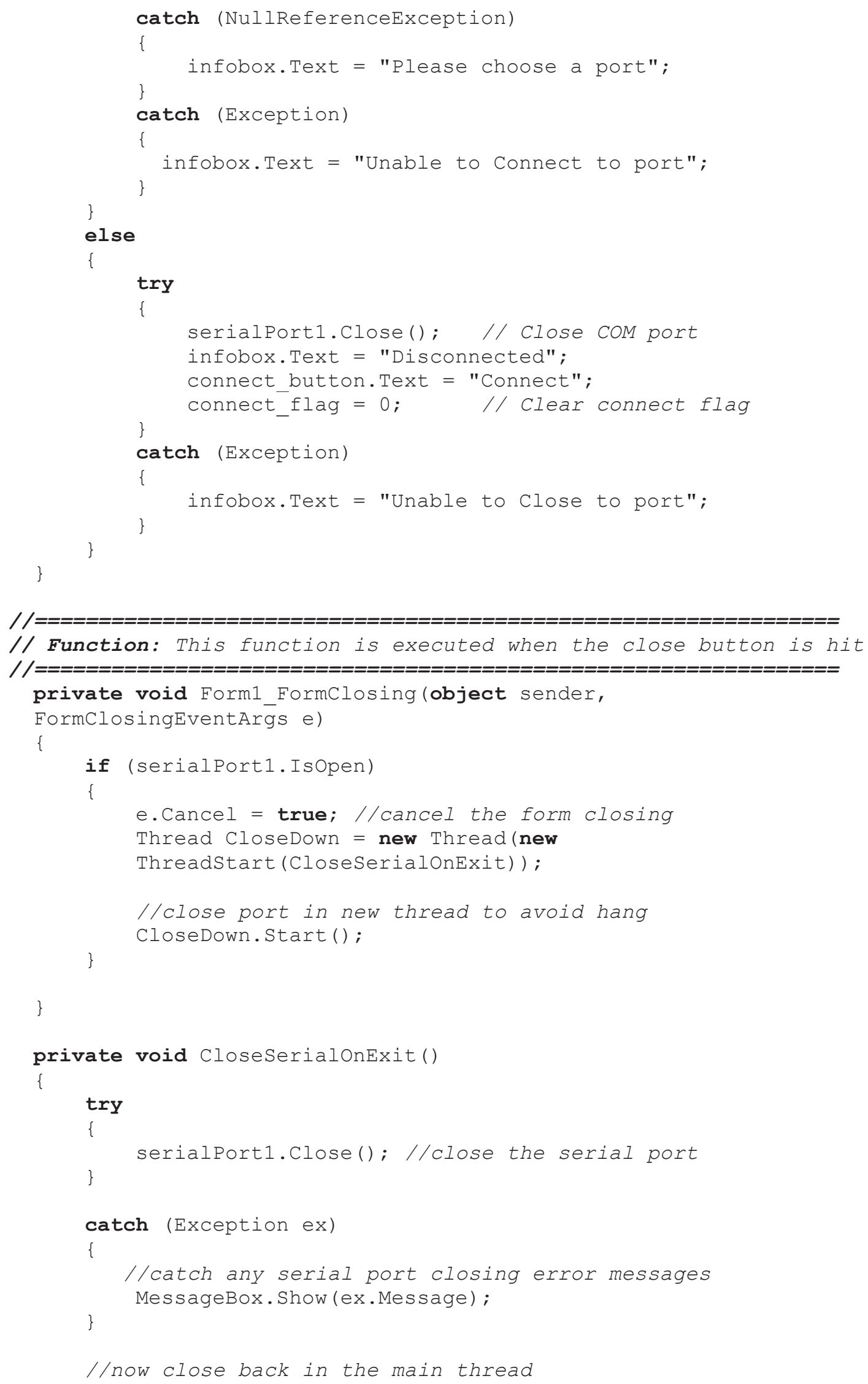


this.Invoke (new EventHandler(NowClose));

\}

private void NowClose (object sender, EventArgs e)

\{

this.Close(); //now close the form

\}

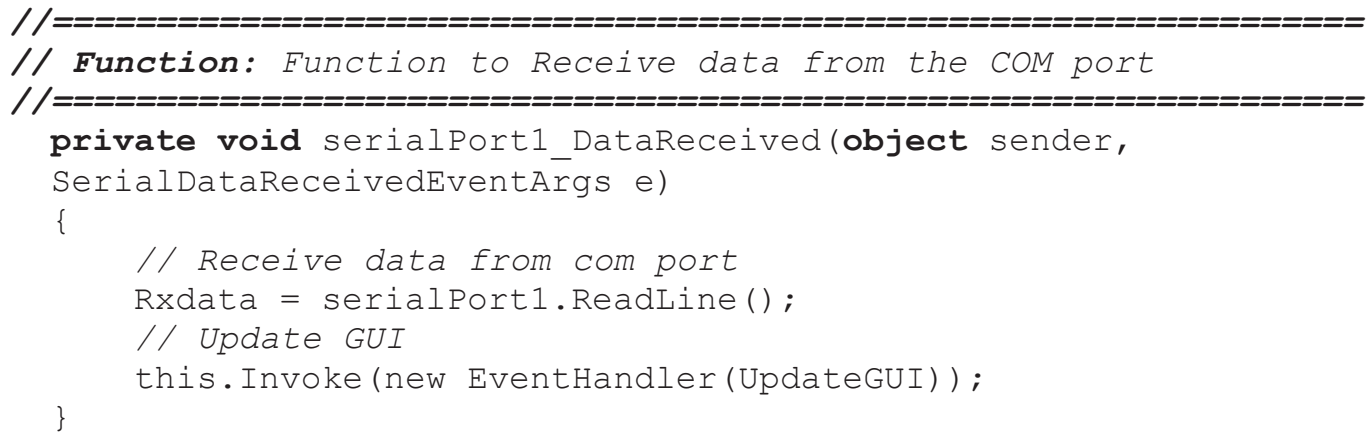




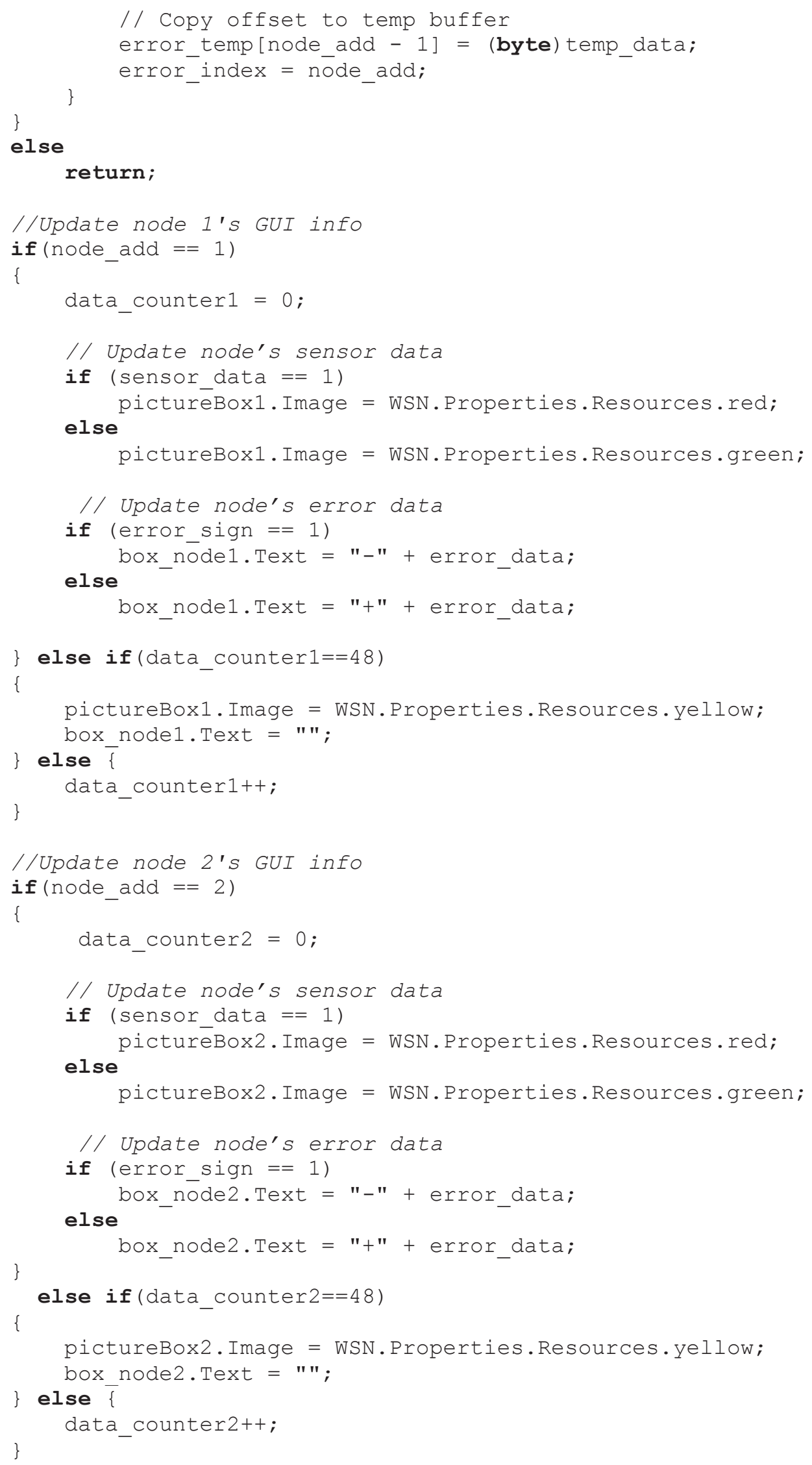




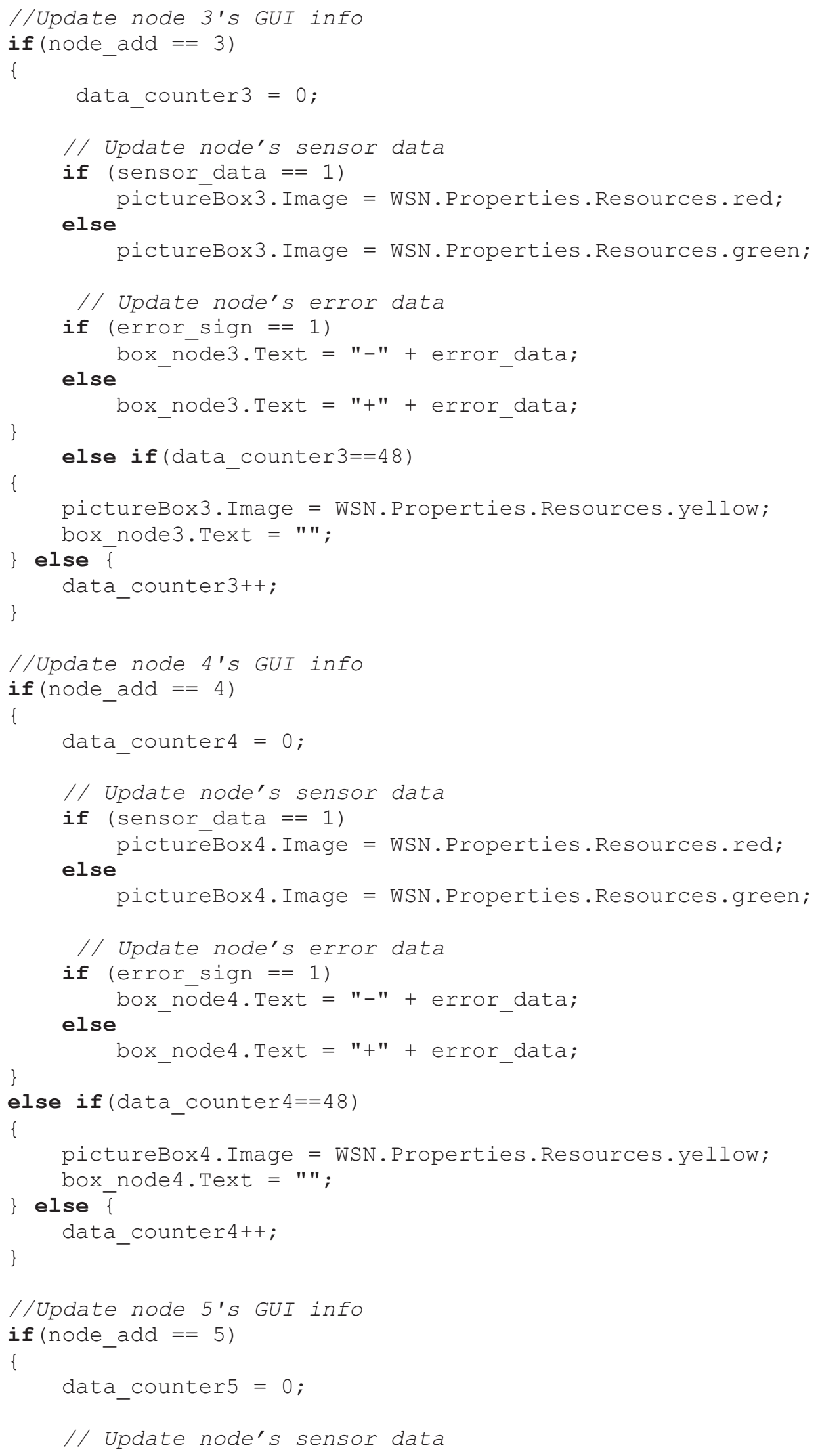




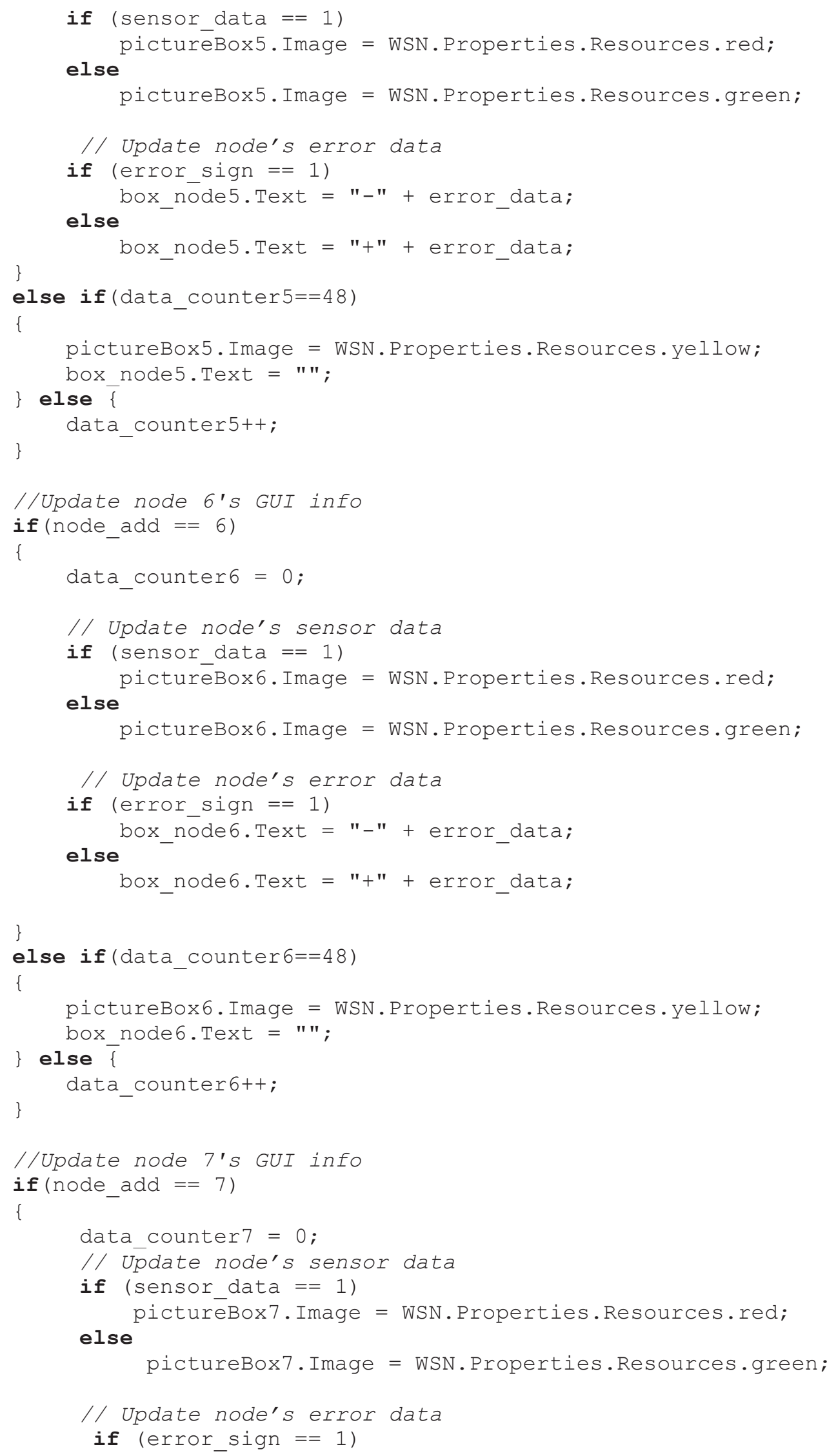


else

box_node7.Text $="-"+$ error_data;

box_node7. Text $="+"+$ error_data;

\}

else if (data_counter $7==48$ )

\{

pictureBox7.Image = WSN.Properties.Resources.yellow;

box node7. Text $="$ ";

\} else $\bar{x}$

\}

data_counter $7++$;

/ Update node 8's GUI info

if (node_add $==8$ )

\{

data_counter $8=0$;

// Update node's sensor data

if ( ensor_data $==1$ )

pictureBox8.Image = WSN.Properties.Resources.red;

else

pictureBox8.Image = WSN.Properties.Resources.green;

// Update node's error data

if (error_sign $==1$ )

box_node8.Text $="-"+$ error_data;

else

box_node8.Text $="+"+$ error_data;

\}

else if (data_counter $8==48$ )

\{

pictureBox 8 .Image = WSN.Properties.Resources.yellow; box node8. Text $=" "$;

\} else $\overline{\{}$

data_counter $8++$;

\}

/ Update node 9's GUI info

if (node_add $==9$ )

\{

data_counter9 $=0$;

// Update node's sensor data

if ( ensor_data $==1$ )

picturēBox9.Image = WSN.Properties.Resources.red;

else

pictureBox9.Image = WSN.Properties.Resources.green;

I/ Update node's error data

if (error_sign $==1$ )

else

box_node9.Text = "-" + error_data;

box_node9.Text $="+"+$ error_data;

\}

else if (data_counter $9==48$ )

\{ 


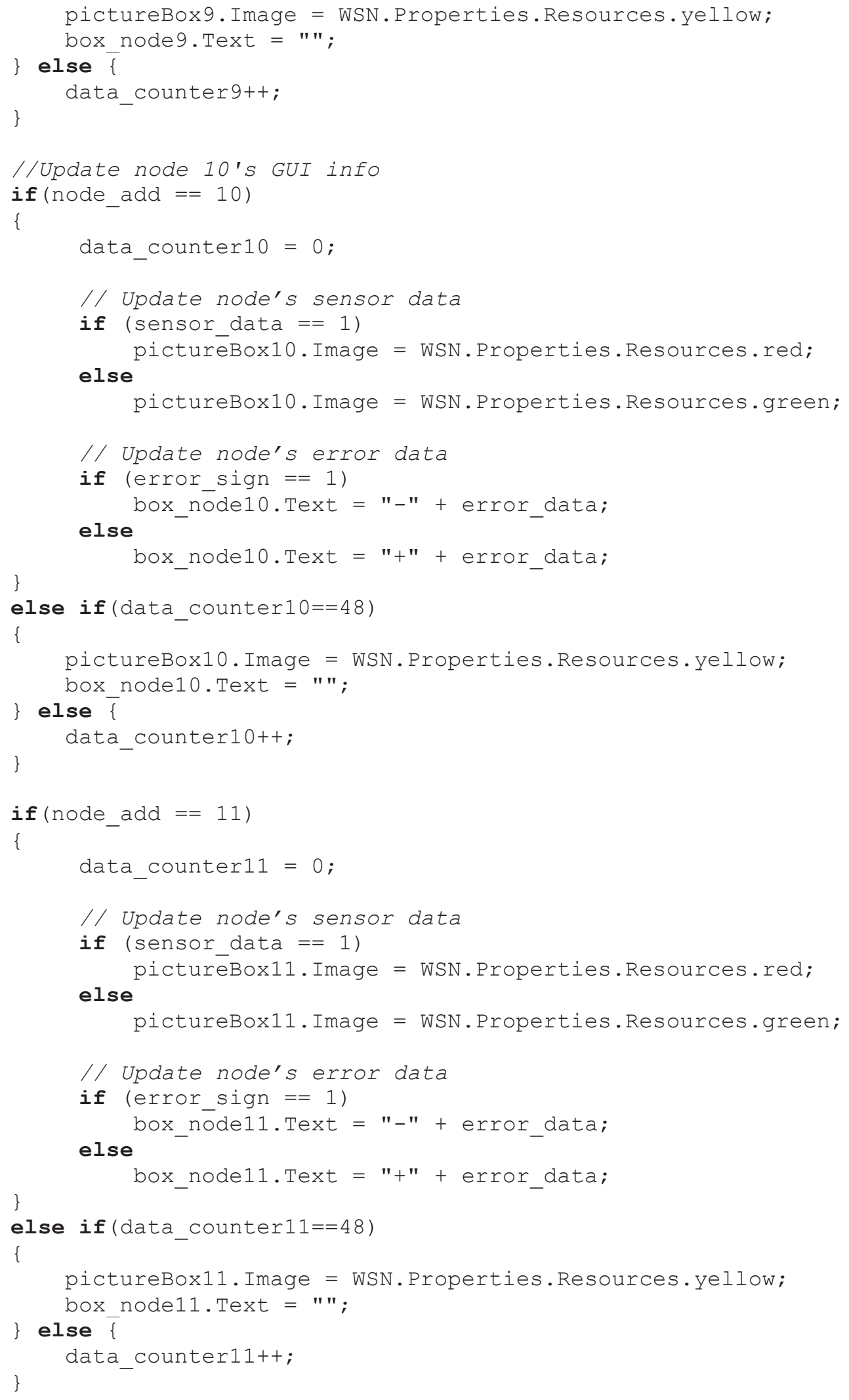




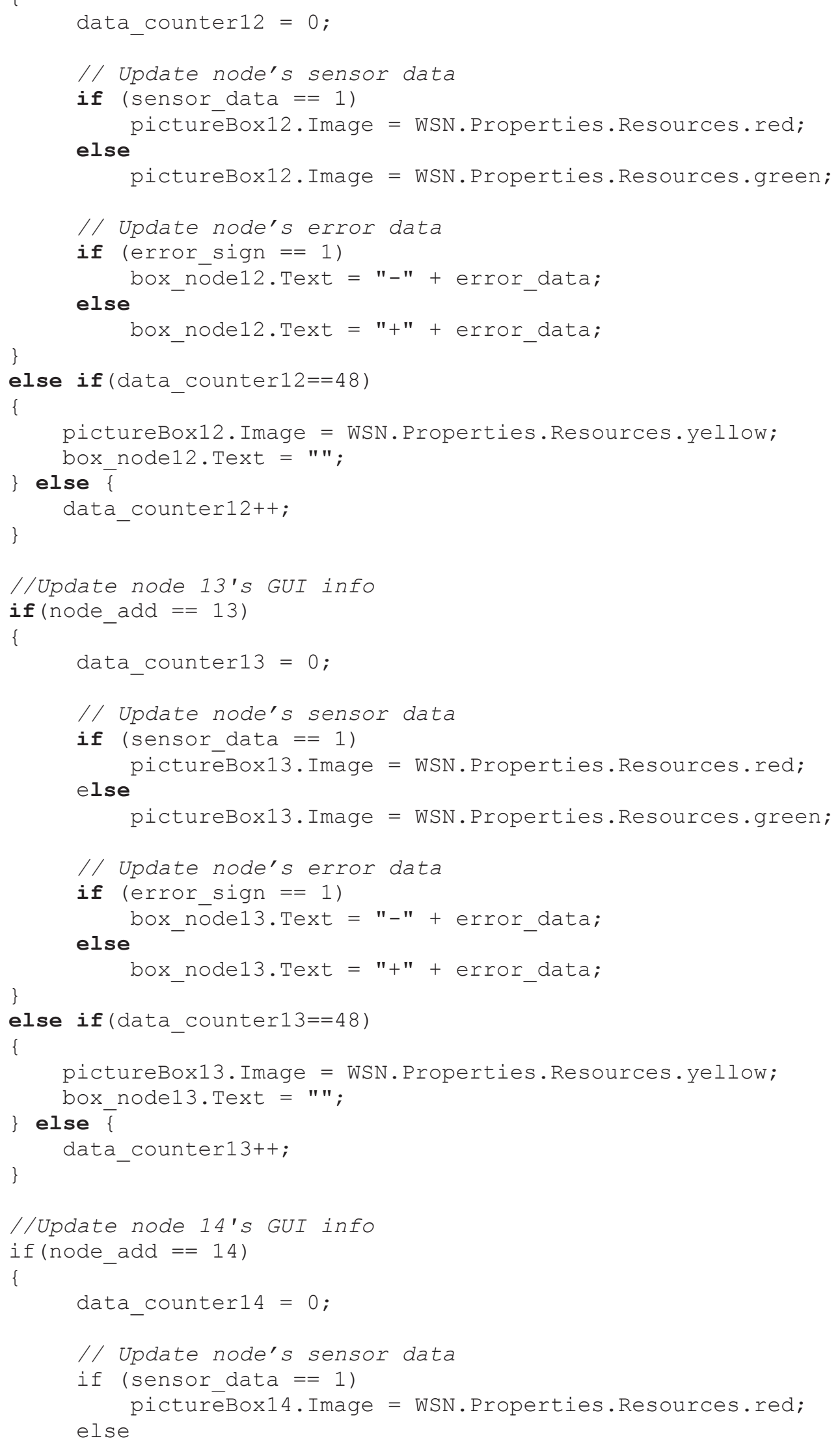




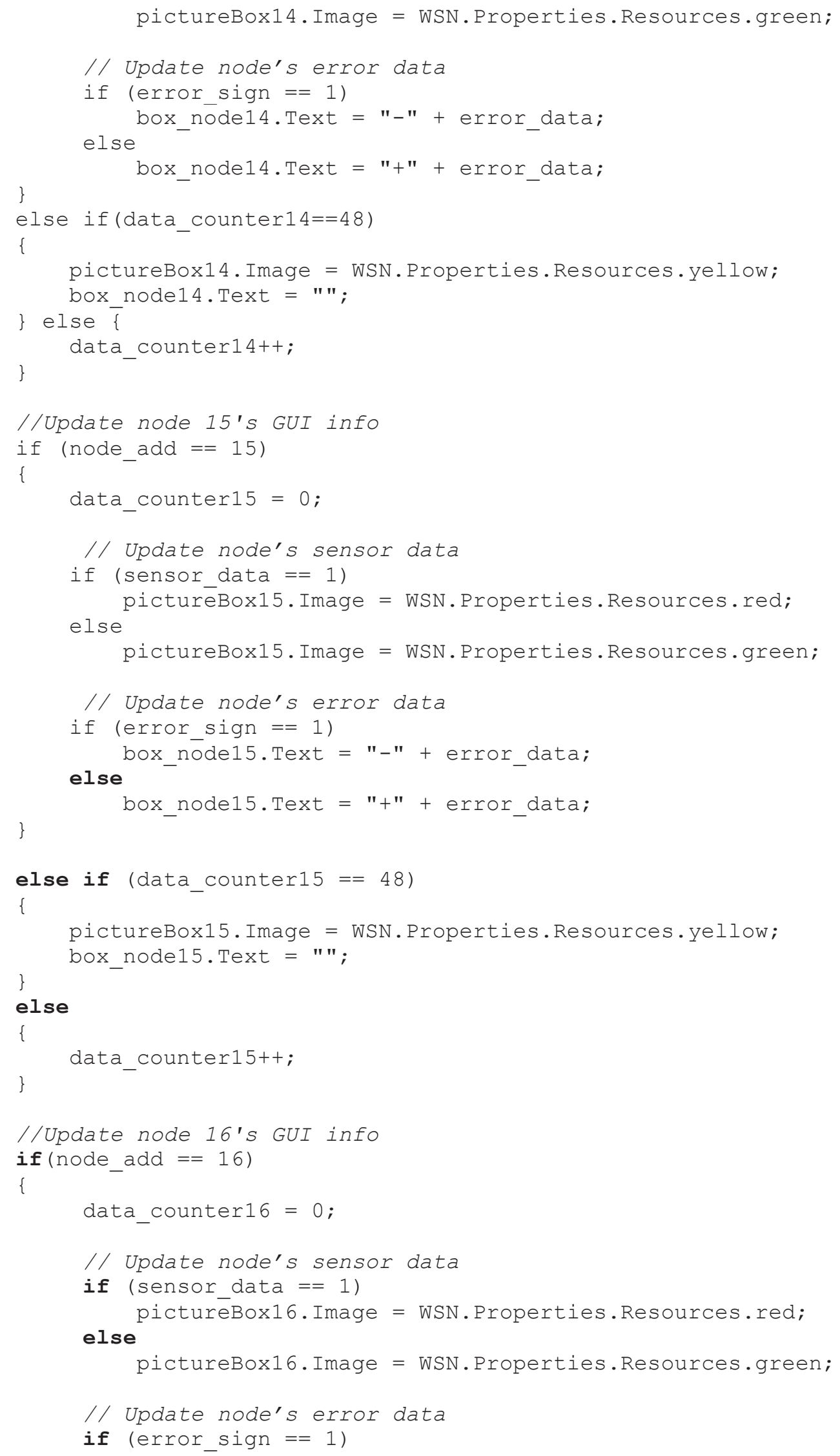




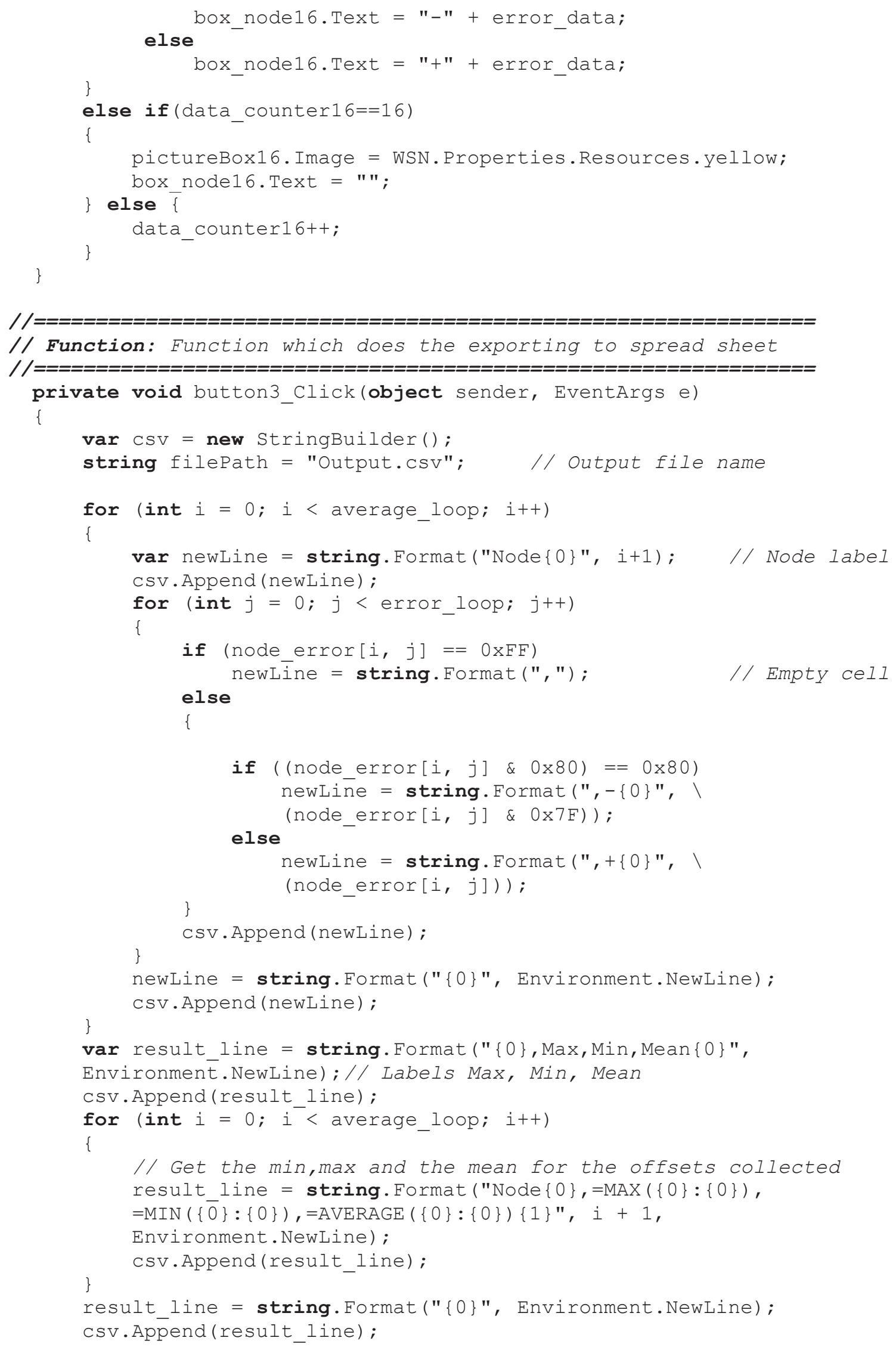


GUI Source Code $\mid 79$

error_loop $=0$;

File. $\bar{W} r i t e A l l$ ext (filePath, csv.ToString()); // Write to the file \} infobox.Text = "File saved"; 